\title{
Electronic structure of kinetic energy driven cuprate superconductors
}

\author{
Shiping Feng1, Huaiming Guo*, Yu Lan, and Li Cheng \\ Department of Physics, Beijing Normal University, Beijing 100875, China
}

In this paper, we review the low energy electronic structure of the kinetic energy driven d-wave cuprate superconductors. We give a general description of the chargespin separation fermion-spin theory, where the constrained electron is decoupled as the gauge invariant dressed holon and spin. In particular, we show that under the decoupling scheme, the charge-spin separation fermion-spin representation is a natural representation of the constrained electron defined in a restricted Hilbert space without double electron occupancy. Based on the charge-spin separation fermionspin theory, we have developed the kinetic energy driven superconducting mechanism, where the superconducting state is controlled by both superconducting gap parameter and quasiparticle coherence. Within this kinetic energy driven superconductivity, we have discussed the low energy electronic structure of the single layer and bilayer cuprate superconductors in both superconducting and normal states, and qualitatively reproduced all main features of the angle-resolved photoemission spectroscopy measurements on the single layer and bilayer cuprate superconductors. We show that the superconducting state in cuprate superconductors is the conventional Bardeen-Cooper-Schrieffer like with the d-wave symmetry, so that the basic Bardeen-Cooper-Schrieffer formalism with the d-wave gap function is still valid in discussions of the low energy electronic structure of cuprate superconductors, although the pairing mechanism is driven by the kinetic energy by exchanging spin excitations. We also show that the well pronounced peak-dip-hump structure of the bilayer cuprate superconductors in the superconducting state and double-peak structure in the normal state are mainly caused by the bilayer splitting.

Keywords: Low energy electronic structure; Kinetic energy driven superconducting mechanism; Cuprate superconductors; Bardeen-Cooper-Schrieffer formalism

\footnotetext{
${ }^{1}$ Corresponding author
} 


\subsection{Introduction}

The parent compounds of cuprate superconductors are the Mott insulators with an antiferromagnetic long-range order, then changing the carrier concentration by ionic substitution or increasing the oxygen content turns these compounds into the superconducting state leaving the antiferromagnetic short-range correlation still intact [1, 2]. It has become clear in the past twenty years that cuprate superconductors are among the most complex systems studied in condensed matter physics 2, 3, 4, 5. The complications arise mainly from (1) a layered crystal structure with one or more $\mathrm{CuO}_{2}$ planes per unit cell separated by insulating layers which leads to a quasi-two-dimensional electronic structure, and (2) extreme sensitivity of the physical properties to the compositions (stoichiometry) which control the carrier density in the $\mathrm{CuO}_{2}$ plane. As a consequence, both experimental investigation [2, 3, 4, 5] and theoretical understanding [6, 7, 8, 9, are extremely difficult. Moreover, the layered crystal structure leads to that cuprate superconductors are highly anisotropic materials, then the electron spectral function $A(\mathbf{k}, \omega)$ is dependent on the in-plane momentum [3, 4, 5]. After twenty years extensive studies, it has been shown that many of the unusual physical properties of cuprate superconductors have often been attributed to particular characteristics of the low energy excitations determined by the electronic structure [3, 4, 5].

Experimentally, by virtue of systematic studies using the angle-resolved photoemission spectroscopy (ARPES), the low energy electronic structure of cuprate superconductors in both superconducting and normal states has been well-established by now [3, 4, [5]. In particular, the information revealed by ARPES experiments has shown that around the nodal and antinodal points of the Brillouin zone contain the essentials of the whole low energy quasiparticle excitation spectrum of cuprate superconductors [3, 4, 5. In the normalstate, the charge carriers doped into the parent compounds first enter into the $\mathbf{k}=[\pi / 2, \pi / 2]$ (in units of inverse lattice constant) point of the Brillouin zone 3, 10, 11, 12. Moreover, the electron spectral function $A(\mathbf{k}, \omega)$ has a flat band form as a function of energy $\omega$ for momentum $\mathbf{k}$ in the vicinity of the $[\pi, 0]$ point just below the Fermi energy, which leads to the unusual quasiparticle dispersion around the $[\pi, 0]$ point with anomalously small changes of electron energy as a function of momentum [3, 10, 11, 12]. However, the lowest energy states are located at the $[\pi, 0]$ point in the superconducting state [4, 5, 13, where the d-wave superconducting gap function is maximal. Furthermore, the improvements in the resolution of the ARPES experiments allowed for an experimental verification of the particle-hole coherence in the superconducting state and Bogoliubovquasiparticle nature of the sharp superconducting quasiparticle peak near the $[\pi, 0]$ point [14, 15. It is striking that in spite of the nonconventional superconducting mechanism and observed exotic magnetic scattering [16, 17, 18, in cuprate superconductors, these ARPES experimental results [14, 15] show that the superconducting coherence of the quasiparticle peak is described by the simple Bardeen-Cooper-Schrieffer (BCS) formalism [19] with the d-wave gap function. Although these common features are observed, there are numerous 
anomalies for different families of cuprate superconductors, which complicate the physical properties of the electronic structure [4, 5. Among these anomalies is the dramatic change in the spectral lineshape around the $[\pi, 0]$ point first observed on the bilayer cuprate superconductor $\mathrm{Bi}_{2} \mathrm{Sr}_{2} \mathrm{CaCu}_{2} \mathrm{O}_{8+\delta}$ in the superconducting state, where a sharp quasiparticle peak develops at the lowest binding energy, followed by a dip and a hump, giving rise to the so-called peak-diphump structure in the electron spectrum [20, 21, 22. Later, this peak-dip-hump structure was also found in $\mathrm{YBa}_{2} \mathrm{Cu}_{3} \mathrm{O}_{7-\delta}$ [23] and in $\mathrm{Bi}_{2} \mathrm{Sr}_{2} \mathrm{Ca}_{2} \mathrm{Cu}_{3} \mathrm{O}_{10+\delta}$ [24. Moreover, although the sharp quasiparticle peaks are identified in the superconducting state along the entire Fermi surface, the peak-dip-hump structure is most strongly developed around the $[\pi, 0]$ point [4, 20, 21, 22, 23, 24. In particular, the similar double-peak structure in the electron spectrum around the $[\pi, 0]$ point has been observed in the bilayer cuprate superconductors in the normal-state [22, 25]. These ARPES experimental measurements raise a question: whether the behavior of the low energy excitations determined by the electronic structure is universal or not.

One of the main concerns in the field of superconductivity in cuprate superconductors is about the origin of the electron pairing. From the experimental side, it has been well established that the antiferromagnetic short-range correlation coexists with the superconducting state in the whole superconducting regime [16, 17, 18. In particular, an impurity-substitution effect on the low energy dynamics of cuprate superconductors has been investigated experimentally [26], which is a magnetic analogue of the isotope effect used for the conventional superconductors. It is shown [26] that the impurity-induced changes in the electron self-energy show a good correspondence to those of magnetic excitations. These experimental results provide a clear link between the charge carrier pairing mechanism and magnetic excitations, and also is an indication of the unconventional superconducting mechanism that is responsible for a high superconducting transition temperature. On the theoretical side, there is an increasing theoretical evidence that purely electronic models can indeed sustain a robust pairing, possibly leading to a high superconducting transition temperature [27, 28. Recently, we 29] have developed a charge-spin separation fermion-spin theory, where the constrained electron operator is decoupled as the gauge invariant dressed holon and spin, with the dressed holon represents the charge degree of freedom together with some effects of the spin configuration rearrangements due to the presence of the doped hole itself, while the spin operator represents the spin degree of freedom. Based on this charge-spin separation fermion-spin theory, we [30] have established a kinetic energy driven superconducting mechanism, where the dressed holon-spin interaction from the kinetic energy term induces the dressed holon pairing state with d-wave symmetry by exchanging spin excitations, then the electron Cooper pairs originating from the dressed holon pairing state are due to the charge-spin recombination, and their condensation reveals the d-wave superconducting ground-state. In particular, this superconducting state is the conventional BCS like with the d-wave symmetry, and is controlled by both superconducting gap function and quasiparticle coherence, then the maximal superconducting transition temperature occurs 
around the optimal doping, and decreases in both underdoped and overdoped regimes 31. Within this framework of the kinetic energy driven superconductivity, we 32, 33, 34, 35, 36, 37. have performed a systematic calculation for the low energy electron spectral function of the single layer and bilayer cuprate superconductors in both superconducting and normal states, and qualitatively reproduced all main features of the ARPES experimental measurements on the single layer and bilayer cuprate superconductors [4, 5]. In this paper, we give a brief review of our recent studies for the low energy electronic structure of the kinetic energy driven d-wave cuprate superconductors [32, 33, 34, 35, 36, 37. We show that in both superconducting and normal states, the spectral weight increases with increasing doping, and decreases with increasing temperatures [32, 33, 34, 35, 36, 37. Furthermore, the superconducting quasiparticles around the $[\pi, 0]$ point disperse very weakly with momentum [35, 37]. In corresponding to this weak dispersions in the superconducting state, the quasiparticle dispersions in the normal state exhibit the flat band around the $[\pi, 0]$ point just below the Fermi energy [34, 36]. Moreover, it is shown that the well pronounced peak-dip-hump structure [37] of the bilayer cuprate superconductors in the superconducting state and double-peak structure [36] in the normal state are mainly caused by the bilayer splitting. In particular, we show that one of the universal features is that the d-wave superconducting state in cuprate superconductors is the conventional BCS like, so that the basic BCS formalism with the d-wave symmetry is still valid in discussions of the low energy electronic structure of cuprate superconductors [32, 33, 35, 37, although the pairing mechanism is driven by the kinetic energy by exchanging spin excitations, and other exotic magnetic properties [16, 17, 18 are beyond the BCS formalism. Our these theoretical results $32,33,34,35,36,37$ also show that the striking behavior of the electronic structure in cuprate superconductors is intriguingly related to the strong coupling between the electron quasiparticles and collective magnetic excitations.

This paper is organized as follows. In section 2, the charge-spin separation fermion-spin theory is presented [29]. It is shown that the charge-spin separation fermion-spin representation is a natural representation of the constrained electron defined in a restricted Hilbert space without double electron occupancy. Within this charge-spin separation fermion-spin theory [29] and kinetic energy driven superconducting mechanism [30, 31, the electronic structure of the single layer cuprate superconductors in both superconducting and normal states [32, 33, 34, 35] is presented in section 3. It is shown that the superconducting coherence of the quasiparticle peak is described by the simple BCS formalism. In section 4, the electronic structure of the bilayer cuprate superconductors [36, 37. is discussed by including the bilayer hopping and bilayer magnetic exchange interaction. It is shown that the electron spectrum is split into the bonding and antibonding components by the bilayer splitting, then the superconducting peak is closely related to the antibonding component, while the hump is mainly formed by the bonding component. Finally, we give a summary and discussions in section 5 . 


\subsection{Charge-spin separation fermion-spin theory}

In cuprate superconductors, the single common feature is the presence of the two-dimensional $\mathrm{CuO}_{2}$ plane 2] as mentioned in section 1, and it seems evident that the nonconventional behaviors are dominated by this plane. Very soon after the discovery of superconductivity in doped cuprates, Anderson argued that the essential physics of the doped $\mathrm{CuO}_{2}$ plane is contained in the Hubbard model or its strong coupling limit, namely the $t-J$ model on a square lattice 6], which includes an antiferromagnetic coupling between localized spins and a kinetic energy term for the hole motion. Furthermore, Anderson proposed a scenario of superconductivity in cuprate superconductors based on the chargespin separation within the two-dimensional $t-J$ model [6], where the internal degrees of freedom of the electron are decoupled as the charge and spin degrees of freedom, then the elementary excitations are collective modes for the charge and spin degrees of freedom, and these collective modes might be responsible for the nonconventional behaviors of cuprate superconductors. Since then, many unusual physical properties of cuprate superconductors are extensively studied along with this line within the two-dimensional $t-J$ model.

The decoupling of the charge and spin degrees of freedom of electron is undoubtedly correct in the one-dimensional interacting electron systems 38, where the charge and spin degrees of freedom are represented by boson operators that describe the excitations of charge-density wave and spin-density wave, respectively. In particular, the typical behavior of the Luttinger liquid, i.e., the absence of the quasiparticle propagation and charge-spin separation, has been demonstrated theoretically within the one-dimensional $t$ - $J$ model [39]. Moreover, the collective modes for the charge and spin degrees of freedom as the real elementary excitations in the one-dimensional cuprates has been observed directly by the ARPES experiment [0. Therefore both theoretical and experimental studies indicate that the existence of the real collective modes for the charge and spin degrees of freedom is common in the one-dimensional interacting electron systems [41]. However, the case in the two-dimensional is very complex since there are many competing degrees of freedom [2, 4, 5. Among the unusual physical properties of cuprate superconductors in the normal state, a hallmark is the charge transport 42, 43, 44, 45, where the conductivity shows a non-Drude behavior at low energies, and is carried by $\delta$ holes, with $\delta$ is the hole doping concentration, while the resistivity exhibits a linear temperature behavior over a wide range of temperatures. This is a strong experimental evidence supporting the notion of the charge-spin separation, since not even conventional electron-electron scattering would show the striking linear rise of scattering rate above the Debye frequency, and if there is no charge-spin separartion, the phonons should affect these properties [7]. Moreover, a compelling evidence for the charge-spin separartion in cuprate superconductors has been found from the experimental test of the Wiedemann-Franz law, where a clear departure from the universal Wiedemann-Franz law for the typical Fermi liquid behavior is observed [46. In this case, a formal theory with the gauge invariant collective modes for the charge and spin degrees of freedom, i.e., the 
issue of whether these collective modes are real, is centrally important for understanding of the physical properties of cuprate superconductors [9]. In this section, we review briefly the charge-spin separation fermion-spin theory [29]. It is shown that if the single occupancy local constraint is treated properly, then the constrained electron in the two-dimensional strong interaction systems can be decoupled completely by introducing the dressed holon and spin, where the dressed holon describes the charge degree of freedom together with some effects of the spin configuration rearrangements due to the presence of the doped hole itself, while the spin operator describes the spin degree of freedom. Furthermore, these dressed holon and spin are gauge invariant, i.e., they are real in the two-dimensional strong interaction systems. In particular, we also show that the charge-spin separation fermion-spin representation is a natural representation for the constrained electron under the decoupling scheme.

We start from the $t-J$ model defined on a square lattice as [6],

$$
H=-t \sum_{i \hat{\eta} \sigma} C_{i \sigma}^{\dagger} C_{i+\hat{\eta} \sigma}+t^{\prime} \sum_{i \hat{\tau} \sigma} C_{i \sigma}^{\dagger} C_{i+\hat{\tau} \sigma}+\mu_{0} \sum_{i \sigma} C_{i \sigma}^{\dagger} C_{i \sigma}+J \sum_{i \hat{\eta}} \mathbf{S}_{i} \cdot \mathbf{S}_{i+\hat{\eta}}
$$

where $\hat{\eta}= \pm \hat{x}, \pm \hat{y}, \hat{\tau}= \pm \hat{x} \pm \hat{y}, C_{i \sigma}^{\dagger}\left(C_{i \sigma}\right)$ is the electron creation (annihilation) operator, $\mathbf{S}_{i}=C_{i}^{\dagger} \vec{\sigma} C_{i} / 2$ is spin operator with $\vec{\sigma}=\left(\sigma_{x}, \sigma_{y}, \sigma_{z}\right)$ as Pauli matrices, and $\mu_{0}$ is the chemical potential. This $t-J$ model (1) is defined in a restricted Hilbert space without double electron occupancy. In this case, there are two ways to implement this requirement: either to solve the $t-J$ model (1) combined with an important single occupancy local constraint,

$$
\sum_{\sigma} C_{i \sigma}^{\dagger} C_{i \sigma} \leq 1
$$

or to introduce the constrained electron operators [47, replacing $C_{i \sigma}$ by $\hat{C}_{i \sigma}=$ $C_{i \sigma}\left(1-n_{i-\sigma}\right)$ with $n_{i \sigma}=C_{i \sigma}^{\dagger} C_{i \sigma}$. We will use both representations in this section.

The on-site single occupancy local constraint (2) is an reflection of the strong on-site Mott-Hubbard Coulombic interaction. There is much evidence that the interactions in cuprate superconductors are dominated by this strong on-site Mott-Hubbard Coulombic interaction [8. Therefore we must deal with the onsite single occupancy local constraint (2) before any analytical calculations 48. For a proper treating the electron single occupancy local constraint (2), we follow the charge-spin separation scheme, and decouple the electron operator in the $t$ - $J$ model (1) as [29, 49],

$$
C_{i \sigma}=h_{i}^{\dagger} a_{i \sigma}
$$

supplemented by the local constraint $\sum_{\sigma} a_{i \sigma}^{\dagger} a_{i \sigma}=1$, where the spinless fermion operator $h_{i}$ keeps track of the charge degree of freedom, while the boson operator $a_{i \sigma}$ keeps track of the spin degree of freedom, then the Hamiltonian (1) can be 
rewritten as,

$$
\begin{aligned}
H & =t \sum_{i \hat{\eta} \sigma} h_{i+\hat{\eta}}^{\dagger} h_{i} a_{i \sigma}^{\dagger} a_{i+\hat{\eta} \sigma}-t^{\prime} \sum_{i \hat{\tau} \sigma} h_{i+\hat{\tau}}^{\dagger} h_{i} a_{i \sigma}^{\dagger} a_{i+\hat{\tau} \sigma}-\mu_{0} \sum_{i} h_{i}^{\dagger} h_{i} \\
& +J \sum_{i \hat{\eta}}\left(1-h_{i}^{\dagger} h_{i}\right) \mathbf{S}_{i} \cdot \mathbf{S}_{i+\hat{\eta}}\left(1-h_{i+\hat{\eta}}^{\dagger} h_{i+\hat{\eta}}\right),
\end{aligned}
$$

with the spin operator $\mathbf{S}_{i}=a_{i}^{\dagger} \vec{\sigma} a_{i} / 2$. In this case, the electron single occupancy local constraint (2) $\sum_{\sigma} C_{i \sigma}^{\dagger} C_{i \sigma}=1-h_{i}^{\dagger} h_{i} \leq 1$ is exactly satisfied, with $n_{i}^{(h)}=h_{i}^{\dagger} h_{i}$ is equal to 1 or 0 . This decoupling scheme is called as the $\mathrm{CP}^{1}$ representation [50], where the elementary charge and spin excitations are called the holon and spinon, respectively. We call such holon and spinon as the bare holon and spinon, respectively, since an extra $U(1)$ gauge degree of freedom related with the single occupancy local constraint $\sum_{\sigma} a_{i \sigma}^{\dagger} a_{i \sigma}=1$ appears, i.e., the $\mathrm{CP}^{1}$ representation is invariant under a local $U(1)$ gauge transformation,

$$
h_{i} \rightarrow h_{i} e^{i \theta_{i}}, \quad a_{i \sigma} \rightarrow a_{i \sigma} e^{i \theta_{i}},
$$

and then all physical quantities should be invariant with respect to this transformation. However, the bare holon $h_{i}$ or bare spinon $a_{i \sigma}$ itself is not gauge invariant, and they are strongly coupled by the $U(1)$ gauge field fluctuations. In other words, these bare holon and spinon are not real.

It has been shown [29, 49, that the $\mathrm{CP}^{1}$ boson $a_{i \sigma}$ together with the local constraint $\sum_{\sigma} a_{i \sigma}^{\dagger} a_{i \sigma}=1$ can be mapped exactly onto the spin representation defined with an additional phase factor. This is because that the empty and doubly occupied spin states have been ruled out due to the constraint $\sum_{\sigma} a_{i \sigma}^{\dagger} a_{i \sigma}=1$, and only the spin-up and spin-down singly occupied spin states are allowed. Therefore the original four-dimensional representation space is reduced to a twodimensional space. Due to the symmetry of the spin-up and spin-down states, $\mid$ occupied $\rangle_{\uparrow}=\left(\begin{array}{l}1 \\ 0\end{array}\right)_{\uparrow}$ and $\mid$ empty $\rangle_{\uparrow}=\left(\begin{array}{l}0 \\ 1\end{array}\right)_{\uparrow}$ are singly-occupied and empty spin-up, while $\mid$ occupied $\rangle_{\downarrow}=\left(\begin{array}{c}0 \\ 1\end{array}\right)_{\downarrow}$ and $\mid$ empty $\rangle_{\downarrow}=\left(\begin{array}{l}1 \\ 0\end{array}\right)_{\downarrow}$ are singlyoccupied and empty spin-down states, respectively, thus the constrained $\mathrm{CP}^{1}$ boson operators $a_{i \uparrow}$ and $a_{i \downarrow}$ can be represented in this reduced two-dimensional space as [29, 49,

$$
\begin{aligned}
& \left.a_{\uparrow}=e^{i \Phi_{\uparrow}} \mid \text { occupied }\right\rangle_{\downarrow \uparrow}\langle\text { occupied }|=e^{i \Phi_{\uparrow}}\left(\begin{array}{cc}
0 & 0 \\
1 & 0
\end{array}\right)=e^{i \Phi_{\uparrow}} S^{-}, \\
& \left.a_{\downarrow}=e^{i \Phi_{\downarrow}} \mid \text { occupied }\right\rangle_{\uparrow}\langle\text { occupied }|=e^{i \Phi_{\downarrow}}\left(\begin{array}{cc}
0 & 1 \\
0 & 0
\end{array}\right)=e^{i \Phi_{\downarrow}} S^{+},
\end{aligned}
$$

with $S^{-}$is the $S^{z}$ lowering operator, while $S^{+}$is the $S^{z}$ raising operator, then the local constraint $\sum_{\sigma} a_{i \sigma}^{\dagger} a_{i \sigma}=S_{i}^{+} S_{i}^{-}+S_{i}^{-} S_{i}^{+}=1$ is exactly satisfied. In this case, the electron decoupling form (3) combined with the electron single 
occupancy local constraint can be expressed as,

$$
C_{i \uparrow}=h_{i}^{\dagger} e^{i \Phi_{i \uparrow}} S_{i}^{-}, \quad C_{i \downarrow}=h_{i}^{\dagger} e^{i \Phi_{i \downarrow}} S_{i}^{+},
$$

then the local $U(1)$ gauge transformation (5) can be rewritten as,

$$
h_{i} \rightarrow h_{i} e^{i \theta_{i}}, \quad \Phi_{i \sigma} \rightarrow \Phi_{i \sigma}+\theta_{i} .
$$

Furthermore, the phase factor $e^{i \Phi_{i \sigma}}$ is induced by the spin configuration rearrangements due to the presence of the doped hole itself, and therefore can be incorporated into the bare holon operator $h_{i}^{\dagger}$. In this case, we can obtain a new transformation from Eq. (7) as 29,

$$
C_{i \uparrow}=h_{i 1}^{\dagger} S_{i}^{-}=h_{i \uparrow}^{\dagger} S_{i}^{-}, \quad C_{i \downarrow}=h_{i 2}^{\dagger} S_{i}^{+}=h_{i \downarrow}^{\dagger} S_{i}^{+},
$$

with the spinful fermion operator $h_{i \sigma}=e^{-i \Phi_{i \sigma}} h_{i}$ represents the charge degree of freedom together with some effects of the spin configuration rearrangement due to the presence of the doped hole itself (dressed holon), while the spin operator $S_{i}$ represents the spin degree of freedom, then electron single occupancy local constraint (2),

$$
\begin{aligned}
\sum_{\sigma} C_{i \sigma}^{\dagger} C_{i \sigma} & =S_{i}^{+} h_{i \uparrow} h_{i \uparrow}^{\dagger} S_{i}^{-}+S_{i}^{-} h_{i \downarrow} h_{i \downarrow}^{\dagger} S_{i}^{+} \\
& =h_{i} h_{i}^{\dagger}\left(S_{i}^{+} S_{i}^{-}+S_{i}^{-} S_{i}^{+}\right)=1-h_{i}^{\dagger} h_{i} \leq 1,
\end{aligned}
$$

is always satisfied in analytical calculations, and the double spinful fermion occupancy $h_{i \sigma}^{\dagger} h_{i-\sigma}^{\dagger}=e^{i \Phi_{i \sigma}} h_{i}^{\dagger} h_{i}^{\dagger} e^{i \Phi_{i-\sigma}}=0$ and $h_{i \sigma} h_{i-\sigma}=e^{-i \Phi_{i \sigma}} h_{i} h_{i} e^{-i \Phi_{i-\sigma}}=$ 0 are ruled out automatically. We call this electron decoupling form (9) as the charge-spin separation fermion-spin transformation [29]. We emphasize that the dressed holon $h_{i \sigma}=e^{-i \Phi_{i \sigma}} h_{i}$ is the spinless fermion $h_{i}$ (bare holon) incorporated the spin cloud $e^{-i \Phi_{i \sigma}}$ (magnetic flux) [51, and is a magnetic dressing. Therefore the 'spin degree of freedom' $1(2)$ or $\uparrow(\downarrow)$ in the dressed holon operator $h_{i 1}=h_{i \uparrow}$ $\left(h_{i 2}=h_{i \downarrow}\right)$ in Eq. (9) is strongly dependent on the spin configuration $S_{i}^{-}\left(S_{i}^{+}\right)$. In particular, these dressed holon and spin are invariant under the local $U(1)$ gauge transformation (8), and therefore all physical quantities from the dressed holon and spin also are invariant with respect to the gauge transformation (8). In this sense, the collective modes for these dressed holon and spin are real and can be interpreted as the physical excitations for the charge and spin degrees of freedom [9]. Although in common sense $h_{i \sigma}$ is not an real spinful fermion operator, it behaves like a spinful fermion. This is followed from that the spinless fermion $h_{i}$ and spin operators $S_{i}^{+}$and $S_{i}^{-}$obey the anticommutation relation and Pauli spin algebra, respectively, it is then easy to show that the spinful fermion $h_{i \sigma}$ also obey the same anticommutation relation as the spinless fermion $h_{i}$. In this charge-spin separation fermion-spin representation (9), the low- 
energy behavior of the $t-J$ model in Eq. (4) can be expressed as [29,

$$
\begin{aligned}
H & =t \sum_{i \hat{\eta}}\left(h_{i+\hat{\eta} \uparrow}^{\dagger} h_{i \uparrow} S_{i}^{+} S_{i+\hat{\eta}}^{-}+h_{i+\hat{\eta} \downarrow}^{\dagger} h_{i \downarrow} S_{i}^{-} S_{i+\hat{\eta}}^{+}\right) \\
& -t^{\prime} \sum_{i \hat{\tau}}\left(h_{i+\hat{\tau} \uparrow}^{\dagger} h_{i \uparrow} S_{i}^{+} S_{i+\hat{\tau}}^{-}+h_{i+\hat{\tau} \downarrow}^{\dagger} h_{i \downarrow} S_{i}^{-} S_{i+\hat{\tau}}^{+}\right) \\
& -\mu_{0} \sum_{i \sigma} h_{i \sigma}^{\dagger} h_{i \sigma}+J_{\mathrm{eff}} \sum_{i \hat{\eta}} \mathbf{S}_{i} \cdot \mathbf{S}_{i+\hat{\eta}}
\end{aligned}
$$

with $J_{\text {eff }}=(1-\delta)^{2} J$, and $\delta=\left\langle h_{i \sigma}^{\dagger} h_{i \sigma}\right\rangle=\left\langle h_{i}^{\dagger} h_{i}\right\rangle$ is the hole doping concentration. As a consequence, the kinetic energy term in the $t-J$ model has been expressed as the dressed holon-spin interaction, which dominates the essential physics of cuprate superconductors, while the magnetic energy term is only to form an adequate spin configuration [7. This also reflects that even the kinetic energy term in the $t$ - $J$ model has strong Coulombic contribution due to the restriction of no doubly occupancy of a given site.

Now we show that the charge-spin separation fermion-spin transformation (9) is a natural representation for the constrained electron under the decoupling scheme. Since the $t-J$ model (1) is defined in the restricted Hilbert space without double electron occupancy, therefore there are two ways to implement this requirement as mentioned above: either to solve the $t$ - $J$ model (1) combined with an important single occupancy local constraint (2) or to introduce the constrained electron operators [47, replacing $C_{i \sigma}$ by $\hat{C}_{i \sigma}=C_{i \sigma}\left(1-n_{i-\sigma}\right)$. In the latter case, the $t-J$ model also can be expressed in terms of these constrained electron operators as 47 ,

$$
H=-t \sum_{i \hat{\eta} \sigma} \hat{C}_{i \sigma}^{\dagger} \hat{C}_{i+\hat{\eta} \sigma}+t^{\prime} \sum_{i \hat{\tau} \sigma} \hat{C}_{i \sigma}^{\dagger} \hat{C}_{i+\hat{\tau} \sigma}+\mu_{0} \sum_{i \sigma} \hat{C}_{i \sigma}^{\dagger} \hat{C}_{i \sigma}+J \sum_{i \hat{\eta}} \mathbf{S}_{i} \cdot \mathbf{S}_{i+\hat{\eta}}
$$

In the constrained electron operator, the operators $C_{i \sigma}^{\dagger}$ and $C_{i \sigma}$ are to be thought of as operating within the full Hilbert space (unprojected Hilbert space), while the constrained electron operator $\hat{C}_{i \sigma}^{\dagger}\left(\hat{C}_{i \sigma}\right)$ does not create (destroy) any doubly occupied sites, and therefore represents physical creation (annihilation) operator acting in the restricted Hilbert space (projected Hilbert subspace) 8]. In particular, these constrained electron operators $\hat{C}_{i \uparrow}$ and $\hat{C}_{i \downarrow}$ can be expressed in a different form as $[8]$,

$$
\begin{aligned}
& \hat{C}_{i \uparrow}=C_{i \uparrow}\left(1-n_{i \downarrow}\right)=C_{i \downarrow} C_{i \downarrow}^{\dagger} C_{i \uparrow}=C_{i \downarrow} S_{i}^{-}, \\
& \hat{C}_{i \downarrow}=C_{i \downarrow}\left(1-n_{i \uparrow}\right)=C_{i \uparrow} C_{i \uparrow}^{\dagger} C_{i \downarrow}=C_{i \uparrow} S_{i}^{+},
\end{aligned}
$$

obviously, where the spin degree of freedom $\downarrow$ ( $\uparrow$ ) in the unprojected operator $C_{i \downarrow}\left(C_{i \uparrow}\right)$ is not a free degree of freedom, and is strongly dependent on the spin configuration $S_{i}^{-}\left(S_{i}^{+}\right)$. Furthermore, this form of the constrained electron operators (13) also show obviously that the spin operators $S_{i}^{+}\left(S_{i}^{-}\right)$represents the spin degree of freedom of the constrained electron, while the unprojected 
operator $C_{i \sigma}$ represents the charge degree of freedom together with some effects of the spin configuration rearrangement due to doping, which is exact same as the charge-spin separation fermion-spin transformation in Eq. (9) if the constrained electron is decoupled according to its charge and spin degrees of freedom. To see this point clearly, the constrained electron operators $\hat{C}_{i \uparrow}$ and $\hat{C}_{i \downarrow}$ in Eq. (13) can be rewritten in terms of a particle-hole transformation $C_{i \sigma} \rightarrow h_{i-\sigma}^{\dagger}$ as,

$$
\hat{C}_{i \uparrow}=h_{i \uparrow}^{\dagger} S_{i}^{-}, \quad \hat{C}_{i \downarrow}=h_{i \downarrow}^{\dagger} S_{i}^{+},
$$

i.e., the creation (annihilation) of the spin-up hole is equivalent to the annihilation (creation) of the spin-down 'electron'. This is why although the assumption of the charge-spin separation in Eq. (9) which underlay that discussions are too radical [8], the essential physics of the constrained electron is kept.

Although the charge-spin separation fermion-spin transformation (9) is a natural representation for the constrained electron (13) under the decoupling scheme, so long as $h_{i}^{\dagger} h_{i}=1, \sum_{\sigma} C_{i \sigma}^{\dagger} C_{i \sigma}=0$, no matter what the values of $S_{i}^{+} S_{i}^{-}$ and $S_{i}^{-} S_{i}^{+}$are, therefore it means that a 'spin' even to an empty site has been assigned. Obviously, this defect is originated from the decoupling approximation. It has been shown [49] that this defect can be cured by introducing a projection operator $P_{i}$, i.e., the electron operator $C_{i \sigma}$ with the single occupancy local constraint (2) can be mapped exactly using the charge-spin separation fermion-spin transformation (9) defined with an additional projection operator $P_{i}$. However, this projection operator is cumbersome to handle in the many cases, and it has been dropped in the actual calculations 229, 30, 31, 32, 34, 35, 36, 37, 33, 49. It has been shown 29, 49, 52 that such treatment leads to errors of the order $\delta$ in counting the number of spin states, which is negligible for small doping. Moreover, the electron single occupancy local constraint still is exactly obeyed even in the mean-field approximation. In particular, the essential physics of the gauge invariant dressed holon and spin is kept [29]. To show this point clearly, we can map electron operator $C_{i \sigma}$ with the electron single occupancy local constraint (2) onto the slave-fermion formulism [29] as $C_{i \sigma}=h_{i}^{\dagger} b_{i \sigma}$ with the local constraint $h_{i}^{\dagger} h_{i}+\sum_{\sigma} b_{i \sigma}^{\dagger} b_{i \sigma}=1$. We can solve the local constraint in the slave-fermion formulism by rewriting the boson operators $b_{i \sigma}$ in terms of the $\mathrm{CP}^{1}$ boson operators $a_{i \sigma}$ as $b_{i \sigma}=a_{i \sigma} \sqrt{1-h_{i}^{\dagger} h_{i}}$ supplemented by the local constraint $\sum_{\sigma} a_{i \sigma}^{\dagger} a_{i \sigma}=1$. As mentioned above, the $\mathrm{CP}^{1}$ boson operators $a_{i \uparrow}$ and $a_{i \downarrow}$ with the constraint $\sum_{\sigma} a_{i \sigma}^{\dagger} a_{i \sigma}=1$ can be identified with the spin lowering and raising operators, respectively, defined with the additional phase factor, therefore the projection operator is approximately related to the dressed holon number operator by $P_{i} \sim \sqrt{1-h_{i \sigma}^{\dagger} h_{i \sigma}}=\sqrt{1-h_{i}^{\dagger} h_{i}}$, and its main role is to remove the spurious spin when there is a holon at a given site $i$ 29, 49, 52, All these are also why the theoretical results $29,30,31,32,34,35,36,37,33,49$, obtained from the $t-J$ model (11) based on the charge-spin separation fermionspin theory (9) are in qualitative agreement with the numerical simulations and experimental observation on cuprate superconductors. 


\subsection{Electronic structure of the single layer cuprate superconductors}

It has been known from the experimental measurements 53 that the maximum superconducting transition temperature varies strongly among different cuprate superconducting compounds. This material dependence has been reduced to the crystal structure, since one or more $\mathrm{CuO}_{2}$ planes per unit cell separated by insulating layers are found for different families of cuprate superconductors, then the crystal structure determines the hybridizations of the $\mathrm{Cu}$ orbital with those of other elements, resulting in different values of hopping integrals within the $\mathrm{CuO}_{2}$ plane and between the $\mathrm{CuO}_{2}$ planes in the unit cell for distinct compounds. This leads to that some subtle structures in the electron spectrum for different families of cuprate superconductors are observed [4, 5]. In this section, we firstly discuss the electronic structure of the single layer cuprate superconductors within the kinetic energy driven superconductivity [31, 32, 34, 35.

\subsubsection{Electronic structure of the single layer cuprate su- perconductors in the superconducting state}

As in the conventional superconductors [19, the superconducting state in cuprate superconductors is also characterized by the electron Cooper pairs [54]. Furthermore, the ARPES measurements [55] show that in the real space the superconducting gap function and pairing force have a range of one lattice spacing. In this case, the order parameter for the electron Cooper pair can be expressed in the charge-spin separation fermion-spin representation as,

$$
\Delta=\left\langle C_{i \uparrow}^{\dagger} C_{i+\hat{\eta} \downarrow}^{\dagger}-C_{i \downarrow}^{\dagger} C_{i+\hat{\eta} \uparrow}^{\dagger}\right\rangle=\left\langle h_{i \uparrow} h_{i+\hat{\eta} \downarrow} S_{i}^{+} S_{i+\hat{\eta}}^{-}-h_{i \downarrow} h_{i+\hat{\eta} \uparrow} S_{i}^{-} S_{i+\hat{\eta}}^{+}\right\rangle .
$$

In the doped regime without the antiferromagnetic long-range order, spins form a disordered spin liquid state, where the spin correlation function $\left\langle S_{i}^{+} S_{i+\hat{\eta}}^{-}\right\rangle=$ $\left\langle S_{i}^{-} S_{i+\hat{\eta}}^{+}\right\rangle$, then the order parameter for the electron Cooper pair in Eq. (15) can be written as,

$$
\Delta=-\left\langle S_{i}^{+} S_{i+\hat{\eta}}^{-}\right\rangle \Delta_{h},
$$

with the dressed holon pairing order parameter,

$$
\Delta_{h}=\left\langle h_{i+\hat{\eta} \downarrow} h_{i \uparrow}-h_{i+\hat{\eta} \uparrow} h_{i \downarrow}\right\rangle,
$$

which shows that the superconducting order parameter is related to the dressed holon pairing amplitude, and is proportional to the number of doped holes, and not to the number of electrons. However, in the extreme low doped regime with the antiferromagnetic long-range order, where the spin correlation function $\left\langle S_{i}^{+} S_{i+\hat{\eta}}^{-}\right\rangle \neq\left\langle S_{i}^{-} S_{i+\hat{\eta}}^{+}\right\rangle$, then the conduct is disrupted by the antiferromagnetic long-range order, and therefore there is no mixing of superconductivity and antiferromagnetic long-range order [56]. Therefore in the following discussions, 
we only focus on the doped regime without the antiferromagnetic long-range order.

Within the mean-field approximation, the $t-J$ model in Eq. (11) can be decoupled as [30,

$$
\begin{aligned}
H_{\mathrm{MFA}} & =H_{t}+H_{J}-2 N Z t \phi_{1} \chi_{1}+2 N Z t^{\prime} \phi_{2} \chi_{2}, \\
H_{t} & =\chi_{1} t \sum_{i \hat{\eta} \sigma} h_{i+\hat{\eta} \sigma}^{\dagger} h_{i \sigma}-\chi_{2} t^{\prime} \sum_{i \hat{\tau} \sigma} h_{i+\hat{\tau} \sigma}^{\dagger} h_{i \sigma}-\mu_{0} \sum_{i \sigma} h_{i \sigma}^{\dagger} h_{i \sigma} \\
H_{J} & =\frac{1}{2} J_{\mathrm{eff}} \epsilon \sum_{i \hat{\eta}}\left(S_{i}^{+} S_{i+\hat{\eta}}^{-}+S_{i}^{-} S_{i+\hat{\eta}}^{+}\right)+J_{\mathrm{eff}} \sum_{i \hat{\eta}} S_{i}^{z} S_{i+\hat{\eta}}^{z} \\
& -t^{\prime} \phi_{2} \sum_{i \hat{\tau}}\left(S_{i}^{+} S_{i+\hat{\tau}}^{-}+S_{i}^{-} S_{i+\hat{\tau}}^{+}\right)
\end{aligned}
$$

with the anisotropic parameter $\epsilon=1+2 t \phi_{1} / J_{\text {eff }}$, the dressed holon's particlehole parameters $\phi_{1}=\left\langle h_{i \sigma}^{\dagger} h_{i+\hat{\eta} \sigma}\right\rangle$ and $\phi_{2}=\left\langle h_{i \sigma}^{\dagger} h_{i+\hat{\tau} \sigma}\right\rangle$, the spin correlation functions $\chi_{1}=\left\langle S_{i}^{+} S_{i+\hat{\eta}}^{-}\right\rangle$and $\chi_{2}=\left\langle S_{i}^{+} S_{i+\hat{\tau}}^{-}\right\rangle, Z$ is the number of the nearest neighbor or second-nearest neighbor sites, and $N$ is the number of sites. Before the discussions of the electronic structure, we now define firstly the dressed holon normal and anomalous Green's functions as,

$$
\begin{aligned}
g\left(i-j, t-t^{\prime}\right) & =\left\langle\left\langle h_{i \sigma}(t) ; h_{j \sigma}^{\dagger}\left(t^{\prime}\right)\right\rangle\right\rangle, \\
\Im\left(i-j, t-t^{\prime}\right) & =\left\langle\left\langle h_{i \downarrow}(t) ; h_{j \uparrow}\left(t^{\prime}\right)\right\rangle\right\rangle, \\
\Im^{\dagger}\left(i-j, t-t^{\prime}\right) & =\left\langle\left\langle h_{i \uparrow}^{\dagger}(t) ; h_{j \downarrow}^{\dagger}\left(t^{\prime}\right)\right\rangle\right\rangle,
\end{aligned}
$$

respectively, and the spin Green's functions as,

$$
\begin{aligned}
D\left(i-j, t-t^{\prime}\right) & =\left\langle\left\langle S_{i}^{+}(t) ; S_{j}^{-}\left(t^{\prime}\right)\right\rangle\right\rangle, \\
D_{z}\left(i-j, t-t^{\prime}\right) & =\left\langle\left\langle S_{i}^{z}(t) ; S_{j}^{z}\left(t^{\prime}\right)\right\rangle\right\rangle,
\end{aligned}
$$

where $\langle\ldots\rangle$ is an average over the ensemble. In the mean-field level, the spin system is an anisotropic away from the half-filling [30], therefore we have defined the two spin Green's function $D\left(i-j, t-t^{\prime}\right)$ and $D_{z}\left(i-j, t-t^{\prime}\right)$ to describe the spin propagations. In the doped regime without the antiferromagnetic longrange order, i.e., $\left\langle S_{i}^{z}\right\rangle=0$, the mean-field theory of the $t$ - $J$ model based on the charge-spin separation fermion-spin theory has been developed [57. within the Kondo-Yamaji decoupling scheme [58, which is a stage one-step further than the Tyablikov's decoupling scheme. Following their discussions [57, we can obtain the the mean-field dressed holon normal Green's function as,

$$
g^{(0)}(\mathbf{k}, \omega)=\frac{1}{\omega-\xi_{\mathbf{k}}},
$$

and mean-field spin Green's functions as,

$$
\begin{aligned}
D^{(0)}(\mathbf{k}, \omega) & =\frac{B_{\mathbf{k}}}{2 \omega_{\mathbf{k}}}\left(\frac{1}{\omega-\omega_{\mathbf{k}}}-\frac{1}{\omega+\omega_{\mathbf{k}}}\right), \\
D_{z}^{(0)}(\mathbf{k}, \omega) & =\frac{B_{z}(\mathbf{k})}{2 \omega_{z}(\mathbf{k})}\left(\frac{1}{\omega-\omega_{z}(\mathbf{k})}-\frac{1}{\omega+\omega_{z}(\mathbf{k})}\right),
\end{aligned}
$$


where $B_{\mathbf{k}}=2 \lambda_{1}\left(A_{1} \gamma_{\mathbf{k}}-A_{2}\right)-\lambda_{2}\left(2 \chi_{2}^{z} \gamma_{\mathbf{k}}^{\prime}-\chi_{2}\right), B_{z}(\mathbf{k})=\epsilon \chi_{1} \lambda_{1}\left(\gamma_{\mathbf{k}}-1\right)-\chi_{2} \lambda_{2}\left(\gamma_{\mathbf{k}}^{\prime}-\right.$ $1), \lambda_{1}=2 Z J_{e f f}, \lambda_{2}=4 Z \phi_{2} t^{\prime}, \gamma_{\mathbf{k}}=(1 / Z) \sum_{\hat{\eta}} e^{i \mathbf{k} \cdot \hat{\eta}}, \gamma_{\mathbf{k}}^{\prime}=(1 / Z) \sum_{\hat{\tau}} e^{i \mathbf{k} \cdot \hat{\tau}}$, $A_{1}=\epsilon \chi_{1}^{z}+\chi_{1} / 2, A_{2}=\chi_{1}^{z}+\epsilon \chi_{1} / 2$, the spin correlation functions $\chi_{1}^{z}=\left\langle S_{i}^{z} S_{i+\hat{\eta}}^{z}\right\rangle$ and $\chi_{2}^{z}=\left\langle S_{i}^{z} S_{i+\hat{\tau}}^{z}\right\rangle$, the mean-field dressed holon excitation spectrum,

$$
\xi_{\mathbf{k}}=\epsilon_{\mathbf{k}}-\mu_{0},
$$

with $\epsilon_{\mathbf{k}}=Z t \chi_{1} \gamma_{\mathbf{k}}-Z t^{\prime} \chi_{2} \gamma_{\mathbf{k}}^{\prime}$, and the mean-field spin excitation spectra,

$$
\begin{aligned}
\omega_{\mathbf{k}}^{2} & =\lambda_{1}^{2}\left[\left(A_{4}-\alpha \epsilon \chi_{1}^{z} \gamma_{\mathbf{k}}-\frac{1}{2 Z} \alpha \epsilon \chi_{1}\right)\left(1-\epsilon \gamma_{\mathbf{k}}\right)\right. \\
& \left.+\frac{1}{2} \epsilon\left(A_{3}-\frac{1}{2} \alpha \chi_{1}^{z}-\alpha \chi_{1} \gamma_{\mathbf{k}}\right)\left(\epsilon-\gamma_{\mathbf{k}}\right)\right] \\
& +\lambda_{2}^{2}\left[\alpha\left(\chi_{2}^{z} \gamma_{\mathbf{k}}^{\prime}-\frac{3}{2 Z} \chi_{2}\right) \gamma_{\mathbf{k}}^{\prime}+\frac{1}{2}\left(A_{5}-\frac{1}{2} \alpha \chi_{2}^{z}\right)\right] \\
& +\lambda_{1} \lambda_{2}\left[\alpha \chi_{1}^{z}\left(1-\epsilon \gamma_{\mathbf{k}}\right) \gamma_{\mathbf{k}}^{\prime}+\frac{1}{2} \alpha\left(\chi_{1} \gamma_{\mathbf{k}}^{\prime}-C_{3}\right)\left(\epsilon-\gamma_{\mathbf{k}}\right)\right. \\
& \left.+\alpha \gamma_{\mathbf{k}}^{\prime}\left(C_{3}^{z}-\epsilon \chi_{2}^{z} \gamma_{\mathbf{k}}\right)-\frac{1}{2} \alpha \epsilon\left(C_{3}-\chi_{2} \gamma_{\mathbf{k}}\right)\right] \\
\omega_{z}^{2}(\mathbf{k}) & =\epsilon \lambda_{1}^{2}\left(\epsilon A_{3}-\frac{1}{Z} \alpha \chi_{1}-\alpha \chi_{1} \gamma_{\mathbf{k}}\right)\left(1-\gamma_{\mathbf{k}}\right)+\lambda_{2}^{2} A_{5}\left(1-\gamma_{\mathbf{k}}^{\prime}\right) \\
& +\lambda_{1} \lambda_{2}\left[\alpha \epsilon C_{3}\left(\gamma_{\mathbf{k}}+\gamma_{\mathbf{k}}^{\prime}-2\right)+\alpha \chi_{2} \gamma_{\mathbf{k}}\left(1-\gamma_{\mathbf{k}}^{\prime}\right)\right],
\end{aligned}
$$

with $A_{3}=\alpha C_{1}+(1-\alpha) /(2 Z), A_{4}=\alpha C_{1}^{z}+(1-\alpha) /(4 Z), A_{5}=\alpha C_{2}+$ $(1-\alpha) /(2 Z)$, and the spin correlation functions $C_{1}=\left(1 / Z^{2}\right) \sum_{\hat{\eta}, \hat{\eta}^{\prime}}\left\langle S_{i+\hat{\eta}}^{+} S_{i+\hat{\eta}^{\prime}}^{-}\right\rangle$, $C_{1}^{z}=\left(1 / Z^{2}\right) \sum_{\hat{\eta}, \hat{\eta}^{\prime}}\left\langle S_{i+\hat{\eta}^{\prime}}^{z} S_{i+\hat{\eta}^{\prime}}^{z}\right\rangle, C_{2}=\left(1 / Z^{2}\right) \sum_{\hat{\tau}, \hat{\tau}^{\prime}}\left\langle S_{i+\hat{\tau}^{\prime}}^{+} S_{i+\hat{\tau}^{\prime}}^{-}\right\rangle$, and $C_{3}=(1 / Z)$ $\sum_{\hat{\tau}}\left\langle S_{i+\hat{\eta}}^{+} S_{i+\hat{\tau}}^{-}\right\rangle, C_{3}^{z}=(1 / Z) \sum_{\hat{\tau}}\left\langle S_{i+\hat{\eta}}^{z} S_{i+\hat{\tau}}^{z}\right\rangle$. In order to satisfy the sum rule of the correlation function $\left\langle S_{i}^{+} S_{i}^{-}\right\rangle=1 / 2$ in the case without the antiferromagnetic long-range order, the important decoupling parameter $\alpha$ has been introduced in the mean-field calculation [57, 58, which can be regarded as the vertex correction.

Within the charge-spin separation fermion-spin theory, we have recently developed the kinetic energy driven mechanism [30, 31, where we have shown that the dressed holon-spin interaction in the $t-J$ model (11) is quite strong, and can induce the dressed holon pairing state (then the electron Cooper pairing state) by exchanging spin excitations in the higher power of the doping concentration $\delta$. Following our previous discussions [30, 31, the self-consistent equations that satisfied by the full dressed holon normal and anomalous Green's functions are obtained as,

$$
\begin{aligned}
g(\mathbf{k}, \omega) & =g^{(0)}(\mathbf{k}, \omega)+g^{(0)}(\mathbf{k}, \omega)\left[\Sigma_{1}^{(h)}(\mathbf{k}, \omega) g(\mathbf{k}, \omega)\right. \\
& \left.-\Sigma_{2}^{(h)}(-\mathbf{k},-\omega) \Im^{\dagger}(\mathbf{k}, \omega)\right], \\
\Im^{\dagger}(\mathbf{k}, \omega) & =g^{(0)}(-\mathbf{k},-\omega)\left[\Sigma_{1}^{(h)}(-\mathbf{k},-\omega) \Im^{\dagger}(-\mathbf{k},-\omega)\right. \\
& \left.+\Sigma_{2}^{(h)}(-\mathbf{k},-\omega) g(\mathbf{k}, \omega)\right],
\end{aligned}
$$


respectively, where the corresponding dressed holon self-energy functions are given by [31,

$$
\begin{aligned}
\Sigma_{1}^{(h)}\left(\mathbf{k}, i \omega_{n}\right) & =\frac{1}{N^{2}} \sum_{\mathbf{p}, \mathbf{p}^{\prime}} \Lambda_{\mathbf{p}+\mathbf{p}^{\prime}+\mathbf{k}}^{2} \\
& \times \frac{1}{\beta} \sum_{i p_{m}} g\left(\mathbf{p}+\mathbf{k}, i p_{m}+i \omega_{n}\right) \Pi\left(\mathbf{p}, \mathbf{p}^{\prime}, i p_{m}\right), \\
\Sigma_{2}^{(h)}\left(\mathbf{k}, i \omega_{n}\right) & =\frac{1}{N^{2}} \sum_{\mathbf{p}, \mathbf{p}^{\prime}} \Lambda_{\mathbf{p}+\mathbf{p}^{\prime}+\mathbf{k}}^{2} \\
& \times \frac{1}{\beta} \sum_{i p_{m}} \Im\left(-\mathbf{p}-\mathbf{k},-i p_{m}-i \omega_{n}\right) \Pi\left(\mathbf{p}, \mathbf{p}^{\prime}, i p_{m}\right),
\end{aligned}
$$

with $\Lambda_{\mathbf{k}}=Z t \gamma_{\mathbf{k}}-Z t^{\prime} \gamma_{\mathbf{k}}^{\prime}$, and the spin bubble,

$$
\Pi\left(\mathbf{p}, \mathbf{p}^{\prime}, i p_{m}\right)=\frac{1}{\beta} \sum_{i p_{m}^{\prime}} D^{(0)}\left(\mathbf{p}^{\prime}, i p_{m}^{\prime}\right) D^{(0)}\left(\mathbf{p}+\mathbf{p}^{\prime}, i p_{m}^{\prime}+i p_{m}\right) .
$$

In the above calculations of the dressed holon self-energies, the spin part has been limited to the mean-field level, i.e., the full spin Green's function $D(\mathbf{k}, \omega)$ in Eq. (27) has been replaced by the mean-field spin Green's function $D^{(0)}(\mathbf{k}, \omega)$ in Eq. (22a), since the theoretical results of the normal state charge transport obtained at this level are consistent with the experimental data of cuprate superconductors in the normal state [29, 59].

In the self-consistent equations (25), since both doping and temperature dependence of the pairing force and dressed holon gap function have been incorporated into the self-energy function $\Sigma_{2}^{(h)}(\mathbf{k}, \omega)$, then the self-energy function $\Sigma_{2}^{(h)}(\mathbf{k}, \omega)$ describes the effective dressed holon pair gap function, while the selfenergy function $\Sigma_{1}^{(h)}(\mathbf{k}, \omega)$ renormalizes the mean-field dressed holon spectrum $\xi_{\mathbf{k}}$ in Eq. (23), and therefore it describes the dressed holon quasiparticle coherence. Furthermore, the self-energy function $\Sigma_{2}^{(h)}(\mathbf{k}, \omega)$ is an even function of $\omega$, while the other self-energy function $\Sigma_{1}^{(h)}(\mathbf{k}, \omega)$ is not. For the convenience, the self-energy function $\Sigma_{1}^{(h)}(\mathbf{k}, \omega)$ can be broken up into its symmetric and antisymmetric parts as, $\Sigma_{1}^{(h)}(\mathbf{k}, \omega)=\Sigma_{1 e}^{(h)}(\mathbf{k}, \omega)+\omega \Sigma_{1 o}^{(h)}(\mathbf{k}, \omega)$, then both $\Sigma_{1 e}^{(h)}(\mathbf{k}, \omega)$ and $\Sigma_{1 o}^{(h)}(\mathbf{k}, \omega)$ are even functions of $\omega$. In this case, the dressed holon quasiparticle coherent weight can be defined as,

$$
\frac{1}{Z_{h F}(\mathbf{k}, \omega)}=1-\Sigma_{1 o}^{(h)}(\mathbf{k}, \omega)
$$

then the dressed holon normal and anomalous Green's functions in Eqs. (25) 
can be rewritten as 30, 31,

$$
\begin{aligned}
g(\mathbf{k}, \omega) & =\frac{\omega Z_{h F}^{-1}(\mathbf{k}, \omega)+\xi_{\mathbf{k}}+\Sigma_{1 e}^{(h)}(\mathbf{k}, \omega)}{\left[\omega Z_{h F}^{-1}(\mathbf{k}, \omega)\right]^{2}-\left[\xi_{\mathbf{k}}+\Sigma_{1 e}^{(h)}(\mathbf{k}, \omega)\right]^{2}-\left[\Sigma_{2}^{(h)}(\mathbf{k}, \omega)\right]^{2}}, \\
\Im^{\dagger}(\mathbf{k}, \omega) & =\frac{-\Sigma_{2}^{(h)}(\mathbf{k}, \omega)}{\left[\omega Z_{h F}^{-1}(\mathbf{k}, \omega)\right]^{2}-\left[\xi_{\mathbf{k}}+\Sigma_{1 e}^{(h)}(\mathbf{k}, \omega)\right]^{2}-\left[\Sigma_{2}^{(h)}(\mathbf{k}, \omega)\right]^{2}} .
\end{aligned}
$$

Recently, a universal high energy anomaly in the electronic structure has been reported in cuprate superconductors by using the ARPES [60], where they found the dispersion anomalies marked by two distinctive high energy scales. From Eqs. (29), we think that the energy dependence of the effective dressed holon pair gap parameter and quasiparticle coherent weight should be considered in the discussions of this high energy anomaly in the electronic structure. However, in this paper, we only focus on the low-energy electronic structure of cuprate superconductors. In this case, the effective dressed holon pair gap function and quasiparticle coherent weight can be discussed in the static limit, i.e., $\bar{\Delta}_{h}(\mathbf{k})=$ $\left.\Sigma_{2}^{(h)}(\mathbf{k}, \omega)\right|_{\omega=0}, Z_{h F}^{-1}(\mathbf{k})=1-\left.\Sigma_{1 o}^{(h)}(\mathbf{k}, \omega)\right|_{\omega=0}$, and $\Sigma_{1 e}^{(h)}(\mathbf{k})=\left.\Sigma_{1 e}^{(h)}(\mathbf{k}, \omega)\right|_{\omega=0}$, then the dressed holon normal and anomalous Green's functions in Eqs. (29) can be expressed explicitly as,

$$
\begin{aligned}
g(\mathbf{k}, \omega) & =Z_{h F}(\mathbf{k})\left(\frac{U_{h \mathbf{k}}^{2}}{\omega-E_{h \mathbf{k}}}+\frac{V_{h \mathbf{k}}^{2}}{\omega+E_{h \mathbf{k}}}\right), \\
\Im^{\dagger}(\mathbf{k}, \omega) & =-Z_{h F}(\mathbf{k}) \frac{\bar{\Delta}_{h Z}(\mathbf{k})}{2 E_{h \mathbf{k}}}\left(\frac{1}{\omega-E_{h \mathbf{k}}}-\frac{1}{\omega+E_{h \mathbf{k}}}\right),
\end{aligned}
$$

with the dressed holon quasiparticle coherence factors,

$$
\begin{aligned}
U_{h \mathbf{k}}^{2} & =\frac{1}{2}\left(1+\frac{\overline{\xi_{\mathbf{k}}}}{E_{h \mathbf{k}}}\right), \\
V_{h \mathbf{k}}^{2} & =\frac{1}{2}\left(1-\frac{\overline{\xi_{\mathbf{k}}}}{E_{h \mathbf{k}}}\right),
\end{aligned}
$$

and

$$
\begin{aligned}
E_{h \mathbf{k}} & =\sqrt{{\overline{\xi_{\mathbf{k}}^{2}}}\left|\bar{\Delta}_{h Z}(\mathbf{k})\right|^{2}}, \\
\overline{\xi_{\mathbf{k}}} & =Z_{h F} \epsilon_{\mathbf{k}}-\mu \\
\bar{\Delta}_{h Z}(\mathbf{k}) & =Z_{h F}(\mathbf{k}) \bar{\Delta}_{h}(\mathbf{k}), \\
\mu & =Z_{h F}\left(\mu_{0}-\Sigma_{1 e}^{(h)}\right),
\end{aligned}
$$

are the dressed holon quasiparticle spectrum, the renormalized dressed holon excitation spectrum, the renormalized dressed holon pair gap function, and the renormalized chemical potential, respectively. Obviously, the dressed holon pairing state is described by the simple Bardeen-Cooper-Schrieffer (BCS) formalism [19, although the pairing mechanism is driven by the kinetic energy by exchanging spin excitations. In particular, this dressed holon quasiparticle is the excitation of a single dressed holon 'adorned' with the attractive interaction between 
paired dressed holons, while the dressed holon quasiparticle coherent weight $Z_{h F}(\mathbf{k})$ reduces the dressed holon (then electron) quasiparticle bandwidth, and therefore the energy scale of the electron quasiparticle band is controlled by the magnetic interaction $J$. Although $Z_{h F}(\mathbf{k})$ and $\Sigma_{1 e}^{(h)}(\mathbf{k})$ still are a function of $\mathbf{k}$, the wave vector dependence may be unimportant [61. It has been shown from the ARPES experiments [4, 5, 13] that in the superconducting state of cuprate superconductors, the lowest energy states are located at the $[\pi, 0]$ point, which indicates that the majority contribution for the electron spectrum comes from the $[\pi, 0]$ point. In this case, the wave vector $\mathbf{k}$ in $Z_{h F}(\mathbf{k})$ and $\Sigma_{1 e}^{(h)}(\mathbf{k})$ can be chosen as $Z_{h F}^{-1}=1-\left.\Sigma_{1 o}^{(h)}(\mathbf{k})\right|_{\mathbf{k}=[\pi, 0]}$ and $\Sigma_{1 e}^{(h)}=\left.\Sigma_{1 e}^{(h)}(\mathbf{k})\right|_{\mathbf{k}=[\pi, 0]}$. In particular, we 31, 32 have shown within the kinetic energy driven superconducting mechanism that the electron Cooper pairs have a dominated d-wave symmetry over a wide range of the doping concentration, around the optimal doping. In this case, the effective dressed holon pair gap function $\bar{\Delta}_{h Z}(\mathbf{k})$ can be expressed explicitly as the d-wave form, $\bar{\Delta}_{h Z}(\mathbf{k})=\bar{\Delta}_{h Z} \gamma_{\mathbf{k}}^{(d)}$ with $\gamma_{\mathbf{k}}^{(d)}=\left(\cos k_{x}-\cos k_{y}\right) / 2$, then the quasiparticle coherent weight and dressed holon effective gap parameter in Eqs. (26a) and (26b) satisfy the following two equations,

$$
\begin{aligned}
1 & =\frac{1}{N^{3}} \sum_{\mathbf{k}, \mathbf{q}, \mathbf{p}} \Lambda_{\mathbf{q}+\mathbf{k}}^{2} \gamma_{\mathbf{k}-\mathbf{p}+\mathbf{q}}^{(a)} \gamma_{\mathbf{k}}^{(a)} \frac{Z_{h F}^{2}}{E_{h \mathbf{k}}} \frac{B_{\mathbf{q}} B_{\mathbf{p}}}{\omega_{\mathbf{q}} \omega_{\mathbf{p}}}\left(\frac{F_{1}^{(1)}(\mathbf{k}, \mathbf{q}, \mathbf{p})}{\left(\omega_{\mathbf{p}}-\omega_{\mathbf{q}}\right)^{2}-E_{h \mathbf{k}}^{2}}\right. \\
& \left.-\frac{F_{1}^{(2)}(\mathbf{k}, \mathbf{q}, \mathbf{p})}{\left(\omega_{\mathbf{p}}+\omega_{\mathbf{q}}\right)^{2}-E_{h \mathbf{k}}^{2}}\right) \\
\frac{1}{Z_{h F}} & =1+\frac{1}{N^{2}} \sum_{\mathbf{q}, \mathbf{p}} \Lambda_{\mathbf{p}+\mathbf{k}_{A}}^{2} Z_{h F} \frac{B_{\mathbf{q}} B_{\mathbf{p}}}{4 \omega_{\mathbf{q}} \omega_{\mathbf{p}}}\left(\frac{F_{2}^{(1)}(\mathbf{q}, \mathbf{p})}{\left(\omega_{\mathbf{p}}-\omega_{\mathbf{q}}-E_{\left.h \mathbf{p}-\mathbf{q}+\mathbf{k}_{A}\right)^{2}}\right.}\right. \\
& +\frac{F_{2}^{(2)}(\mathbf{q}, \mathbf{p})}{\left(\omega_{\mathbf{p}}-\omega_{\mathbf{q}}+E_{\left.h \mathbf{p}-\mathbf{q}+\mathbf{k}_{A}\right)^{2}}\right.}+\frac{F_{2}^{(3)}(\mathbf{q}, \mathbf{p})}{\left(\omega_{\mathbf{p}}+\omega_{\mathbf{q}}-E_{\left.h \mathbf{p}-\mathbf{q}+\mathbf{k}_{A}\right)^{2}}\right.} \\
& \left.+\frac{F_{2}^{(4)}(\mathbf{q}, \mathbf{p})}{\left(\omega_{\mathbf{p}}+\omega_{\mathbf{q}}+E_{h \mathbf{p}-\mathbf{q}+\mathbf{k}_{A}}\right)^{2}}\right)
\end{aligned}
$$

respectively, where $\mathbf{k}_{A}=[\pi, 0]$, and

$$
\begin{aligned}
& F_{1}^{(1)}(\mathbf{k}, \mathbf{q}, \mathbf{p})=\left(\omega_{\mathbf{p}}-\omega_{\mathbf{q}}\right)\left[n_{B}\left(\omega_{\mathbf{q}}\right)-n_{B}\left(\omega_{\mathbf{p}}\right)\right]\left[1-2 n_{F}\left(E_{h \mathbf{k}}\right)\right] \\
& +E_{h \mathbf{k}}\left[n_{B}\left(\omega_{\mathbf{p}}\right) n_{B}\left(-\omega_{\mathbf{q}}\right)+n_{B}\left(\omega_{\mathbf{q}}\right) n_{B}\left(-\omega_{\mathbf{p}}\right)\right] \\
& F_{1}^{(2)}(\mathbf{k}, \mathbf{q}, \mathbf{p})=\left(\omega_{\mathbf{p}}+\omega_{\mathbf{q}}\right)\left[n_{B}\left(-\omega_{\mathbf{p}}\right)-n_{B}\left(\omega_{\mathbf{q}}\right)\right]\left[1-2 n_{F}\left(E_{h \mathbf{k}}\right)\right] \\
& +E_{h \mathbf{k}}\left[n_{B}\left(\omega_{\mathbf{p}}\right) n_{B}\left(\omega_{\mathbf{q}}\right)+n_{B}\left(-\omega_{\mathbf{p}}\right) n_{B}\left(-\omega_{\mathbf{q}}\right)\right] \text {, } \\
& F_{2}^{(1)}(\mathbf{q}, \mathbf{p})=n_{F}\left(E_{h \mathbf{p}-\mathbf{q}+\mathbf{k}_{A}}\right)\left[n_{B}\left(\omega_{\mathbf{q}}\right)-n_{B}\left(\omega_{\mathbf{p}}\right)\right] \\
& \text { - } n_{B}\left(\omega_{\mathbf{p}}\right) n_{B}\left(-\omega_{\mathbf{q}}\right) \text {, } \\
& F_{2}^{(2)}(\mathbf{q}, \mathbf{p})=n_{F}\left(E_{h \mathbf{p}-\mathbf{q}+\mathbf{k}_{A}}\right)\left[n_{B}\left(\omega_{\mathbf{p}}\right)-n_{B}\left(\omega_{\mathbf{q}}\right)\right] \\
& \text { - } n_{B}\left(\omega_{\mathbf{q}}\right) n_{B}\left(-\omega_{\mathbf{p}}\right) \text {, }
\end{aligned}
$$




$$
\begin{aligned}
F_{2}^{(3)}(\mathbf{q}, \mathbf{p}) & =n_{F}\left(E_{h \mathbf{p}-\mathbf{q}+\mathbf{k}_{A}}\right)\left[n_{B}\left(\omega_{\mathbf{q}}\right)-n_{B}\left(-\omega_{\mathbf{p}}\right)\right] \\
& +n_{B}\left(\omega_{\mathbf{p}}\right) n_{B}\left(\omega_{\mathbf{q}}\right) \\
F_{2}^{(4)}(\mathbf{q}, \mathbf{p}) & =n_{F}\left(E_{h \mathbf{p}-\mathbf{q}+\mathbf{k}_{A}}\right)\left[n_{B}\left(-\omega_{\mathbf{q}}\right)-n_{B}\left(\omega_{\mathbf{p}}\right)\right] \\
& +n_{B}\left(-\omega_{\mathbf{p}}\right) n_{B}\left(-\omega_{\mathbf{q}}\right) .
\end{aligned}
$$

These two equations (33a) and (33b) must be solved simultaneously with other self-consistent equations [30, 31],

$$
\begin{aligned}
& \phi_{1}=\frac{1}{2 N} \sum_{\mathbf{k}} \gamma_{\mathbf{k}} Z_{h F}\left(1-\frac{\overline{\xi_{\mathbf{k}}}}{E_{h \mathbf{k}}} \tanh \left[\frac{1}{2} \beta E_{h \mathbf{k}}\right]\right), \\
& \phi_{2}=\frac{1}{2 N} \sum_{\mathbf{k}} \gamma_{\mathbf{k}}^{\prime} Z_{h F}\left(1-\frac{\overline{\xi_{\mathbf{k}}}}{E_{h \mathbf{k}}} \tanh \left[\frac{1}{2} \beta E_{h \mathbf{k}}\right]\right), \\
& \delta=\frac{1}{2 N} \sum_{\mathbf{k}} Z_{h F}\left(1-\frac{\overline{\xi_{\mathbf{k}}}}{E_{h \mathbf{k}}} \tanh \left[\frac{1}{2} \beta E_{h \mathbf{k}}\right]\right), \\
& \chi_{1}=\frac{1}{N} \sum_{\mathbf{k}} \gamma_{\mathbf{k}} \frac{B_{\mathbf{k}}}{2 \omega_{\mathbf{k}}} \operatorname{coth}\left[\frac{1}{2} \beta \omega_{\mathbf{k}}\right] \text {, } \\
& \chi_{2}=\frac{1}{N} \sum_{\mathbf{k}} \gamma_{\mathbf{k}}^{\prime} \frac{B_{\mathbf{k}}}{2 \omega_{\mathbf{k}}} \operatorname{coth}\left[\frac{1}{2} \beta \omega_{\mathbf{k}}\right] \\
& C_{1}=\frac{1}{N} \sum_{\mathbf{k}} \gamma_{\mathbf{k}}^{2} \frac{B_{\mathbf{k}}}{2 \omega_{\mathbf{k}}} \operatorname{coth}\left[\frac{1}{2} \beta \omega_{\mathbf{k}}\right] \\
& C_{2}=\frac{1}{N} \sum_{\mathbf{k}} \gamma_{\mathbf{k}}^{\prime 2} \frac{B_{\mathbf{k}}}{2 \omega_{\mathbf{k}}} \operatorname{coth}\left[\frac{1}{2} \beta \omega_{\mathbf{k}}\right], \\
& C_{3}=\frac{1}{N} \sum_{\mathbf{k}} \gamma_{\mathbf{k}} \gamma_{\mathbf{k}}^{\prime} \frac{B_{\mathbf{k}}}{2 \omega_{\mathbf{k}}} \operatorname{coth}\left[\frac{1}{2} \beta \omega_{\mathbf{k}}\right] \text {, } \\
& \frac{1}{2}=\frac{1}{N} \sum_{\mathbf{k}} \frac{B_{\mathbf{k}}}{2 \omega_{\mathbf{k}}} \operatorname{coth}\left[\frac{1}{2} \beta \omega_{\mathbf{k}}\right], \\
& \chi_{1}^{z}=\frac{1}{N} \sum_{\mathbf{k}} \gamma_{\mathbf{k}} \frac{B_{z}(\mathbf{k})}{2 \omega_{z}(\mathbf{k})} \operatorname{coth}\left[\frac{1}{2} \beta \omega_{z}(\mathbf{k})\right], \\
& \chi_{2}^{z}=\frac{1}{N} \sum_{\mathbf{k}} \gamma_{\mathbf{k}}^{\prime} \frac{B_{z}(\mathbf{k})}{2 \omega_{z}(\mathbf{k})} \operatorname{coth}\left[\frac{1}{2} \beta \omega_{z}(\mathbf{k})\right], \\
& C_{1}^{z}=\frac{1}{N} \sum_{\mathbf{k}} \gamma_{\mathbf{k}}^{2} \frac{B_{z}(\mathbf{k})}{2 \omega_{z}(\mathbf{k})} \operatorname{coth}\left[\frac{1}{2} \beta \omega_{z}(\mathbf{k})\right], \\
& C_{3}^{z}=\frac{1}{N} \sum_{\mathbf{k}} \gamma_{\mathbf{k}} \gamma_{\mathbf{k}}^{\prime} \frac{B_{z}(\mathbf{k})}{2 \omega_{z}(\mathbf{k})} \operatorname{coth}\left[\frac{1}{2} \beta \omega_{z}(\mathbf{k})\right],
\end{aligned}
$$

then all the order parameters, decoupling parameter $\alpha$, and chemical potential $\mu$ are determined by the self-consistent calculation [30, 31, 32. With above discussions, the dressed holon pair gap function can be obtained in terms of the 
dressed holon anomalous Green's function (30b) as,

$$
\Delta_{h}(\mathbf{k})=-\frac{1}{\beta} \sum_{i \omega_{n}} \Im^{\dagger}\left(\mathbf{k}, i \omega_{n}\right)=\frac{1}{2} Z_{h F} \frac{\bar{\Delta}_{h Z}(\mathbf{k})}{E_{h \mathbf{k}}} \tanh \left[\frac{1}{2} \beta E_{h \mathbf{k}}\right],
$$

then the dressed holon pair order parameter in Eq. (17) can be evaluated explicitly from this dressed holon pair gap function as,

$$
\Delta_{h}=\frac{2}{N} \sum_{\mathbf{k}}\left[\gamma_{\mathbf{k}}^{(d)}\right]^{2} \frac{Z_{h F} \bar{\Delta}_{h Z}^{(a)}}{E_{h \mathbf{k}}} \tanh \left[\frac{1}{2} \beta E_{h \mathbf{k}}\right] .
$$

In our previous discussions [30, 31, 32, we have shown that the dressed holon pairing state originating from the kinetic energy term by exchanging spin excitations also leads to form the electron Cooper pairing state. In this case, the electron normal and anomalous Green's functions $G\left(i-j, t-t^{\prime}\right)=\left\langle\left\langle C_{i \sigma}(t) ; C_{j \sigma}^{\dagger}\left(t^{\prime}\right)\right\rangle\right\rangle$ and $\Gamma^{\dagger}\left(i-j, t-t^{\prime}\right)=\left\langle\left\langle C_{i \uparrow}^{\dagger}(t) ; C_{j \downarrow}^{\dagger}\left(t^{\prime}\right)\right\rangle\right\rangle$ are the convolutions of the spin Green's function and dressed holon normal and anomalous Green's functions, and can be obtained in terms of the mean-field spin Green's function (22a) and dressed holon normal and anomalous Green's functions (30a) and (30b) as,

$$
\begin{aligned}
G(\mathbf{k}, \omega) & =\frac{1}{N} \sum_{\mathbf{p}} Z_{h F} \frac{B_{\mathbf{p}}}{4 \omega_{\mathbf{p}}}\left\{\operatorname { c o t h } [ \frac { 1 } { 2 } \beta \omega _ { \mathbf { p } } ] \left(\frac{U_{h \mathbf{p}+\mathbf{k}}^{2}}{\omega+E_{h \mathbf{p}+\mathbf{k}}-\omega_{\mathbf{p}}}\right.\right. \\
& \left.+\frac{U_{h \mathbf{p}+\mathbf{k}}^{2}}{\omega+E_{h \mathbf{p}+\mathbf{k}}+\omega_{\mathbf{p}}}+\frac{V_{h \mathbf{p}+\mathbf{k}}^{2}}{\omega-E_{h \mathbf{p}+\mathbf{k}}+\omega_{\mathbf{p}}}+\frac{V_{h \mathbf{p}+\mathbf{k}}^{2}}{\omega-E_{h \mathbf{p}+\mathbf{k}}-\omega_{\mathbf{p}}}\right) \\
& +\tanh \left[\frac{1}{2} \beta E_{h \mathbf{p}+\mathbf{k}}\right]\left(\frac{U_{h \mathbf{p}+\mathbf{k}}^{2}}{\omega+E_{h \mathbf{p}+\mathbf{k}}+\omega_{\mathbf{p}}}-\frac{U_{h \mathbf{p}+\mathbf{k}}^{2}}{\omega+E_{h \mathbf{p}+\mathbf{k}}-\omega_{\mathbf{p}}}\right. \\
& \left.\left.+\frac{V_{h \mathbf{p}+\mathbf{k}}^{2}}{\omega-E_{h \mathbf{p}+\mathbf{k}}-\omega_{\mathbf{p}}}-\frac{V_{h \mathbf{p}+\mathbf{k}}^{2}}{\omega-E_{h \mathbf{p}+\mathbf{k}}+\omega_{\mathbf{p}}}\right)\right\}, \\
\Gamma^{\dagger}(\mathbf{k}, \omega) & \left.=\frac{1}{N} \sum_{\mathbf{p}} Z_{h F} \frac{\bar{\Delta} h Z}{2 E_{h \mathbf{p}+\mathbf{k}}}+\mathbf{\mathbf { k }}\right) \frac{B_{\mathbf{p}}}{4 \omega_{\mathbf{p}}}\left\{\operatorname { c o t h } [ \frac { 1 } { 2 } \beta \omega _ { \mathbf { p } } ] \left(\frac{1}{\omega-E_{h \mathbf{p}+\mathbf{k}}-\omega_{\mathbf{p}}}\right.\right. \\
& \left.+\frac{1}{\omega-E_{h \mathbf{p}+\mathbf{k}}+\omega_{\mathbf{p}}}-\frac{1}{\omega+E_{h \mathbf{p}+\mathbf{k}}+\omega_{\mathbf{p}}}-\frac{1}{\omega+E_{h \mathbf{p}+\mathbf{k}}-\omega_{\mathbf{p}}}\right) \\
& +\tanh \left[\frac{1}{2} \beta E_{h \mathbf{p}+\mathbf{k}}\right]\left(\frac{1}{\omega-E_{h \mathbf{p}+\mathbf{k}}-\omega_{\mathbf{p}}}-\frac{1}{\omega-E_{h \mathbf{p}+\mathbf{k}}+\omega_{\mathbf{p}}}\right. \\
& \left.\left.-\frac{1}{\omega+E_{h \mathbf{p}+\mathbf{k}}+\omega_{\mathbf{p}}}+\frac{1}{\omega+E_{h \mathbf{p}+\mathbf{k}}-\omega_{\mathbf{p}}}\right)\right\}
\end{aligned}
$$

respectively, these convolutions of the spin Green's function and dressed holon normal and anomalous Green's functions reflect the charge-spin recombination [7, then the electron spectral function and superconducting gap function are 
obtained from the above electron normal and anomalous Green's functions as,

$$
\begin{aligned}
A(\mathbf{k}, \omega) & =-2 \operatorname{Im} G(\mathbf{k}, \omega) \\
& =2 \pi \frac{1}{N} \sum_{\mathbf{p}} Z_{h F} \frac{B_{\mathbf{p}}}{4 \omega_{\mathbf{p}}}\left\{\operatorname { c o t h } ( \frac { 1 } { 2 } \beta \omega _ { \mathbf { p } } ) \left[U_{h \mathbf{p}+\mathbf{k}}^{2} \delta\left(\omega+E_{h \mathbf{p}+\mathbf{k}}-\omega_{\mathbf{p}}\right)\right.\right. \\
& +U_{h \mathbf{p}+\mathbf{k}}^{2} \delta\left(\omega+E_{h \mathbf{p}+\mathbf{k}}+\omega_{\mathbf{p}}\right)+V_{h \mathbf{p}+\mathbf{k}}^{2} \delta\left(\omega-E_{h \mathbf{p}+\mathbf{k}}+\omega_{\mathbf{p}}\right) \\
& \left.+V_{h \mathbf{p}+\mathbf{k}}^{2} \delta\left(\omega-E_{h \mathbf{p}+\mathbf{k}}-\omega_{\mathbf{p}}\right)\right]+\tanh \left(\frac{1}{2} \beta E_{h \mathbf{p}+\mathbf{k}}\right) \\
& \times\left[U_{h \mathbf{p}+\mathbf{k}}^{2} \delta\left(\omega+E_{h \mathbf{p}+\mathbf{k}}+\omega_{\mathbf{p}}\right)-U_{h \mathbf{p}+\mathbf{k}}^{2} \delta\left(\omega+E_{h \mathbf{p}+\mathbf{k}}-\omega_{\mathbf{p}}\right)\right. \\
& \left.\left.+V_{h \mathbf{p}+\mathbf{k}}^{2} \delta\left(\omega-E_{h \mathbf{p}+\mathbf{k}}-\omega_{\mathbf{p}}\right)-V_{h \mathbf{p}+\mathbf{k}}^{2} \delta\left(\omega-E_{h \mathbf{p}+\mathbf{k}}+\omega_{\mathbf{p}}\right)\right]\right\} \\
\Delta(\mathbf{k}) & =-\frac{1}{\beta} \sum_{i \omega_{n}} \Gamma^{\dagger}\left(\mathbf{k}, i \omega_{n}\right) \\
& =\frac{-1}{N} \sum_{\mathbf{p}} \frac{Z_{h F} \bar{\Delta}_{h Z}(\mathbf{p}-\mathbf{k})}{2 E_{h \mathbf{p}-\mathbf{k}}} \tanh \left[\frac{1}{2} \beta E_{h \mathbf{p}-\mathbf{k}}\right] \frac{B_{\mathbf{p}}}{2 \omega_{\mathbf{p}}} \operatorname{coth}\left[\frac{1}{2} \beta \omega_{\mathbf{p}}\right], \quad
\end{aligned}
$$

which shows that the symmetry of the electron Cooper pair is completely determined by the symmetry of the dressed holon pair. With the above superconducting gap function (40), the superconducting gap parameter in Eq. (15) is obtained as $\Delta=-\chi_{1} \Delta_{h}$. Since both dressed holon (then electron) pairing gap parameter and pairing interaction in cuprate superconductors are doping dependent, then the experimental observed doping dependence of the superconducting gap parameter should be an effective superconducting gap parameter $\bar{\Delta} \sim-\chi_{1} \bar{\Delta}_{h}$. On the other hand, the electron quasiparticle coherent weight $Z_{F}$ can be obtained from the sum rule for the electron spectral function $A(\mathbf{k}, \omega)$ in Eq. (39) as,

$$
Z_{F}=\int_{-\infty}^{\infty} \frac{d \omega}{2 \pi} A(\mathbf{k}, \omega)=\frac{1}{N} \sum_{\mathbf{p}} Z_{h F} \frac{B_{\mathbf{p}}}{2 \omega_{\mathbf{p}}} \operatorname{coth}\left(\frac{\beta \omega_{\mathbf{p}}}{2}\right)=\frac{1}{2} Z_{h F} .
$$

In Fig. 1, we plot (a) the electron quasiparticle coherent weight $Z_{F}\left(T_{c}\right)$, (b) the effective superconducting gap parameter $\bar{\Delta}$ at temperature $T=0.002 J$, and (c) the superconducting transition temperature $T_{c}$ as a function of the doping concentration $\delta$ for parameters $t / J=2.5$ and $t^{\prime} / t=0.3$. For comparison, the corresponding experimental results (inset) of the quasiparticle coherent weight in the $[\pi, 0]$ point [13, superconducting gap parameter [62, and superconducting transition temperature [63] as a function of the doping concentration are also shown in Fig. 1(a), 1(b), and 1(c), respectively. Our results show that the quasiparticle coherent weight grows linearly with the doping concentration, i.e., $Z_{F} \propto \delta$, which together with the superconducting gap parameter defined in Eq. (15) show that only $\delta$ number of coherent doped carriers are recovered in the superconducting state, consistent with the picture of a doped Mott insulator with $\delta$ holes 6 . In this case, the superconducting state of cuprate superconductors is controlled by both superconducting gap function and superconducting 

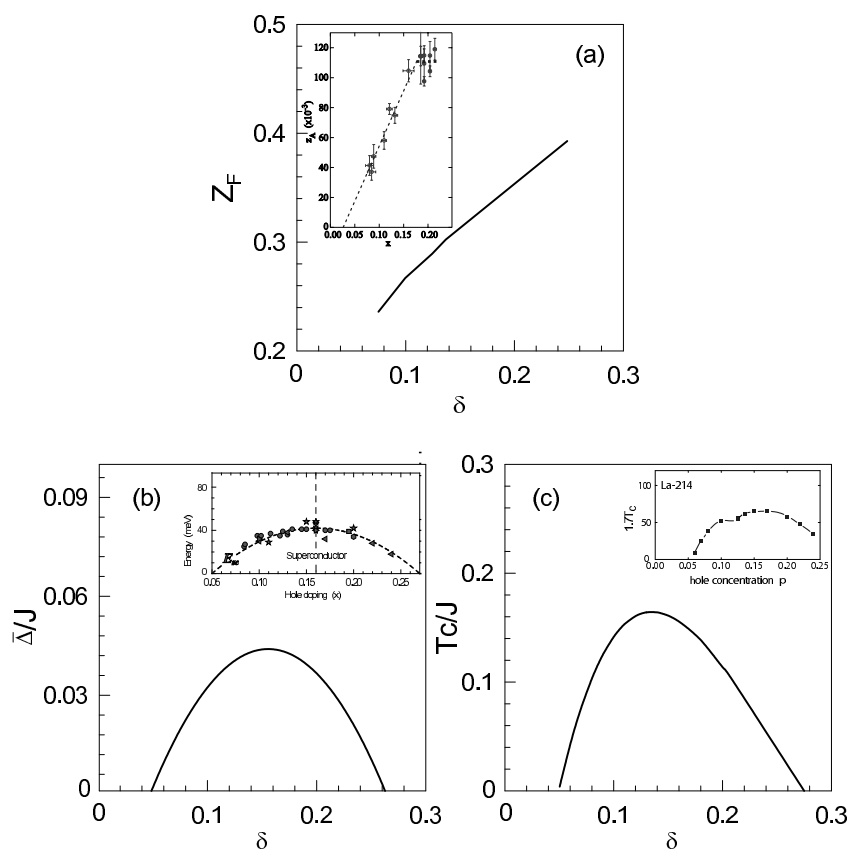

Figure 1.1: (a) The electron quasiparticle coherent weight $Z_{F}\left(T_{c}\right)$ in the $[\pi, 0]$ point, (b) the effective superconducting gap parameter $\bar{\Delta}$ at $T=0.002 \mathrm{~J}$, and (c) the superconducting transition temperature $T_{c}$ as a function of the doping concentration for $t / J=2.5$ and $t^{\prime} / t=0.3$. Inset: the corresponding experimental results of cuprate superconductors taken from Refs. [13, 62] and 63, respectively.

quasiparticle coherence [13, 31, 32]. Since the dressed holons (then electrons) interact by exchanging spin excitations and that this interaction is attractive. This attractive interaction leads to form the dressed holon pairs (then electron Cooper pairs). The perovskite parent compound of doped cuprate superconductors is a Mott insulator, when holes are doped into this insulator, there is a gain in the kinetic energy per hole proportional to $t$ due to hopping, but at the same time, the spin correlation is destroyed, costing an energy of approximately $J$ per site, therefore the doped holes into the Mott insulator can be considered as a competition between the kinetic energy $(\delta t)$ and magnetic energy $(J)$, and the magnetic energy decreases with increasing doping. In the underdoped and optimally doped regimes, the magnetic energy is rather too large, and the dressed holon (then electron) attractive interaction by exchanging spin excitations is also rather strong to form the dressed holon pairs (then electron Cooper pairs) for the most dressed holons (then electrons), therefore the number of the dressed holon pairs (then electron Cooper pairs) and superconducting transition temperature [65] are proportional to the hole doping concentration. However, in the 


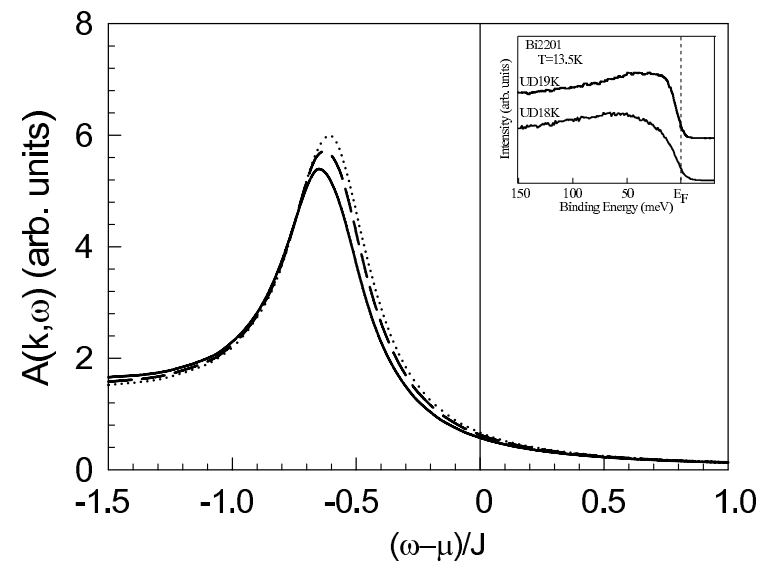

Figure 1.2: The electron spectral function $A(\mathbf{k}, \omega)$ at the $[\pi, 0]$ point in the superconducting state with $\delta=0.09$ (solid line), $\delta=0.12$ (dashed line), and $\delta=0.15$ (dotted line) at $T=0.002 J$ for $t / J=2.5$ and $t^{\prime} / t=0.3$. Inset: the corresponding experimental result of the single layer cuprate superconductor $\mathrm{Bi}_{2} \mathrm{Sr}_{2} \mathrm{CuO}_{6+\delta}$ in the superconducting state taken from Ref. [66].

overdoped regime, the magnetic energy is relatively small, and the dressed holon (then electron) attractive interaction by exchanging spin excitations is also relatively weak, in this case, not all dressed holons (then electrons) can be bounden as dressed holon pairs (then electron Cooper pairs) by the weak attractive interaction, and therefore the number of the dressed holon pairs (then electron Cooper pairs) and superconducting transition temperature 63 decrease with increasing doping. Using an reasonably estimative value of $J \sim 800 \mathrm{~K}$ to $1200 \mathrm{~K}$ in cuprate superconductors 2, the superconducting transition temperature in the optimal doping is $\mathrm{T}_{c} \approx 0.165 J \approx 132 \mathrm{~K} \sim 198 \mathrm{~K}$, in qualitative agreement with the experimental data 63.

Now we discuss the electronic structure of the single layer cuprate superconductors in the superconducting state. We have performed a calculation for the electron spectral function $A(\mathbf{k}, \omega)$ in Eq. (39), and the results of $A(\mathbf{k}, \omega)$ in the $[\pi, 0]$ point with $\delta=0.09$ (solid line), $\delta=0.12$ (dashed line), and $\delta=0.15$ (dotted line) at $T=0.002 J$ for $t / J=2.5$ and $t^{\prime} / t=0.3$ are plotted in Fig. 2 in comparison with the corresponding experimental result of the single layer cuprate superconductor $\mathrm{Bi}_{2} \mathrm{Sr}_{2} \mathrm{CuO}_{6+\delta}$ in the superconducting state 66] (inset). Obviously, (1) there is a sharp superconducting quasiparticle peak near the electron Fermi energy in the $[\pi, 0]$ point, and the position of the superconducting quasiparticle peak in $\delta=0.15$ is located at $\omega_{\text {peak }} \approx 0.6 J \approx 0.042 \mathrm{eV} \sim 0.06 \mathrm{eV}$, which is qualitatively consistent with $\omega_{\text {peak }} \approx 0.035 \mathrm{eV}$ observed 66] in the underdoped single layer cuprate superconductor $\mathrm{Bi}_{2} \mathrm{Sr}_{2} \mathrm{CuO}_{6+\delta}$ in the superconducting state; (2) The electron spectrum in the superconducting state is doping dependent. With increasing the doping concentration, the weight of the 


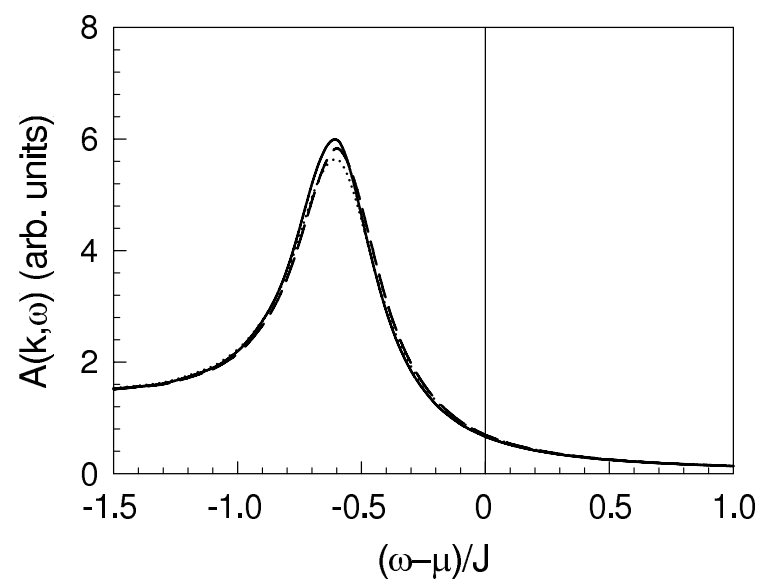

Figure 1.3: The electron spectral function $A(\mathbf{k}, \omega)$ at the $[\pi, 0]$ point in the superconducting state in $\delta=0.15$ with $T=0.002 \mathrm{~J}$ (solid line), $T=0.10 \mathrm{~J}$ (dashed line), and $T=0.15 J$ (dotted line) for $t / J=2.5$ and $t^{\prime} / t=0.3$.

superconducting quasiparticle peaks increases; (3) The position of the superconducting quasiparticle peak moves to the Fermi energy with increasing doping 66. Furthermore, we have discussed the temperature dependence of the electron spectrum in the superconducting state, and the results of $A(\mathbf{k}, \omega)$ in the $[\pi, 0]$ point with $\delta=0.15$ at $T=0.002 J$ (solid line), $T=0.10 J$ (dashed line), and $T=0.15 J$ (dotted line) for $t / J=2.5$ and $t^{\prime} / t=0.3$ are plotted in Fig. 3 . It is shown that the spectral weight decreases as temperature is increased. These theoretical results under the kinetic energy driven superconducting mechanism is in qualitative agreement with the experimental data of the single layer cuprate superconductors in the superconducting state [4, 66, 67].

For the further understanding of the superconducting coherence of the sharp quasiparticle peak around the $[\pi, 0]$ point, we have made a series of calculations for $A(\mathbf{k}, \omega)$ around the $[\pi, 0]$ point, and the results show that the sharp superconducting quasiparticle peak persists in a very large momentum space region around the $[\pi, 0]$ point. To show this point clearly, we plot the positions of the lowest energy superconducting quasiparticle peaks in $A(\mathbf{k}, \omega)$ as a function of momentum along the direction $[0,0] \rightarrow[\pi, 0] \rightarrow[2 \pi, 0]$ at $\delta=0.15$ with $T=0.002 J$ for $t / J=2.5$ and $t^{\prime} / t=0.3$ in Fig. 4 in comparison with the corresponding experimental result of the single layer cuprate superconductor $\mathrm{Bi}_{2} \mathrm{Sr}_{2} \mathrm{CuO}_{6+\delta}$ in the superconducting state 68. (inset). Our results show that the sharp low energy superconducting quasiparticle peaks around the $[\pi, 0]$ point disperse very weakly with momentum, which also is qualitatively consistent with these obtained from ARPES experimental measurements on the single layer cuprate superconductors in the superconducting state [4, 67, 68].

A natural question is why the superconducting coherence of the superconducting quasiparticle peak in cuprate superconductors can be described quali- 




Figure 1.4: The positions of the lowest energy superconducting quasiparticle peaks in $A(\mathbf{k}, \omega)$ as a function of momentum along the direction $[0,0] \rightarrow[\pi, 0] \rightarrow$ $[2 \pi, 0]$ with $\delta=0.15$ at $T=0.002 J$ for $t / J=2.5$ and $t^{\prime} / t=0.3$. Inset: the corresponding experimental result of the single layer cuprate superconductor $\mathrm{Bi}_{2} \mathrm{Sr}_{2} \mathrm{CuO}_{6+\delta}$ in the superconducting state taken from Ref. 68.

tatively in the framework of the kinetic energy driven superconductivity. The reason is that the superconducting state in the kinetic energy driven superconductivity is the conventional BCS like withe the d-wave symmetry [32, 35. This can be understood from the electron normal and anomalous Green's functions in Eqs. (38a) and (38b). Since spins center around the $[\pi, \pi]$ point in the meanfield level [30, 31, then the main contributions for the spins comes from the $[\pi, \pi]$ point. In this case, the electron normal and anomalous Green's functions in Eqs. (38a) and (38b) can be approximately reduced in terms of $\omega_{\mathbf{p}=[\pi, \pi]} \sim 0$ and the self-consistent equation (35i) as the simple BCS formalism with the d-wave gap function,

$$
\begin{aligned}
G(\mathbf{k}, \omega) & \approx Z_{F}\left(\frac{U_{\mathbf{k}}^{2}}{\omega-E_{\mathbf{k}}}+\frac{V_{\mathbf{k}}^{2}}{\omega+E_{\mathbf{k}}}\right), \\
\Gamma^{\dagger}(\mathbf{k}, \omega) & \approx Z_{F} \frac{\bar{\Delta}_{h Z}(\mathbf{k})}{2 E_{\mathbf{k}}}\left(\frac{1}{\omega-E_{\mathbf{k}}}+\frac{1}{\omega+E_{\mathbf{k}}}\right),
\end{aligned}
$$

where the electron quasiparticle coherence factors,

$$
\begin{aligned}
& U_{\mathbf{k}}^{2} \approx V_{h \mathbf{k}+\mathbf{k}_{A F}}^{2}, \\
& V_{\mathbf{k}}^{2} \approx U_{h \mathbf{k}+\mathbf{k}_{A F}}^{2},
\end{aligned}
$$

and electron quasiparticle spectrum $E_{\mathbf{k}} \approx E_{h \mathbf{k}+\mathbf{k}_{A F}}$, with $\mathbf{k}_{A F}=[\pi, \pi]$, i.e., the hole-like dressed holon quasiparticle coherence factors $V_{h \mathbf{k}}$ and $U_{h \mathbf{k}}$ in Eqs. (31) and hole-like dressed holon quasiparticle spectrum $E_{h \mathbf{k}}$ in Eq. (32a) have been transferred into the electron quasiparticle coherence factors $U_{\mathbf{k}}$ and $V_{\mathbf{k}}$ and electron quasiparticle spectrum $E_{\mathbf{k}}$, respectively, by the convolutions of 
the spin Green's function and dressed holon Green's functions, which means that the dressed holon pairs condense with the d-wave symmetry in a wide range of the doping concentration, then the electron Cooper pairs originating from the dressed holon pairing state are due to the charge-spin recombination, and their condensation automatically gives the electron quasiparticle character. This electron quasiparticle is the excitation of a single electron 'dressed' with the attractive interaction between paired electrons. This is why the basic BCS formalism with the d-wave gap function [19] is still valid in discussions of the doping dependence of the superconducting gap parameter and superconducting transition temperature, and superconducting coherence of the quasiparticle peak [14, 15, although the pairing mechanism is driven by the kinetic energy by exchanging spin excitations, and other exotic magnetic scattering [16, 17, 18] is beyond the BCS formalism.

As we have known that the quasiparticle is defined as in Fermi liquid theory and gives a measure of how the quasiparticle is to being a free electron 69. The basis for BCS theory of the conventional superconductivity is the formation first of a Fermi liquid, i.e., a quantum coherent state. Then one looks for strong interactions which crossover to an attractive pairing interaction in some high angular momentum channel 70. In particular, the quasiparticle coherent weight $Z_{F}=1$ in the simple BCS model for the conventional superconductors [19]. However, in the kinetic energy driven superconducting mechanism, although the superconducting coherence of the quasiparticle peak is described by the simple BCS formalism with the d-wave gap function, the pairing mechanism is driven by the kinetic energy by exchanging spin excitations as mentioned above, which reflects that the strong electron correlation

does not suppress superconductivity, but rather is to favor it because the main ingredient was identified into the pairing mechanism not involving phonons as in the conventional superconductors, but the internal spin degrees of freedom. We [71] have also calculated the superconducting quasiparticle coherent weight $Z_{F}$ in Eq. (41) with different momenta, and the results show that the overall spectral weight in cuprate superconductors is heavily reduced $\left(Z_{F} \ll 1\right)$ by the strong electron correlation, in qualitative agreement with these obtained from the variational Monte Carlo simulations [72. All these also are a consequence of the fact that the electron states of cuprate superconductors are restricted in the Hilbert subspace without double electron occupancy (projected Hilbert subspace), where although the dressed holon quasiparticle coherent weight $Z_{h F}$ in Eq. (28) obeys the usual perturbation theory identities relating it to the dressed holon self-energy function, the value of the true superconducting quasiparticle coherent weight $Z_{F}$ is obtained in terms of the charge-spin recombination.

\subsubsection{Electronic structure of the single layer cuprate su- perconductors in the normal state}

In correspondence with the above discussions of the electronic structure of the single layer cuprate superconductors in the superconducting state, we now turn to discuss the electronic structure of the single layer cuprate superconductors in 
the normal state. In the normal state, the dressed holon pairing order parameter $\Delta_{h}=0$ (then superconducting gap parameter $\Delta=0$ ), then the dressed holon normal Green's function in Eq. (30a) is reduced as,

$$
g(\mathbf{k}, \omega)=\frac{Z_{h F}}{\omega-\overline{\xi_{\mathbf{k}}}}
$$

It has been shown from the ARPES experiments [3, 10, 11, 12] that in the normal-state, the lowest energy states are located at the $[\pi / 2, \pi / 2]$ point, which indicates that the majority contribution for the electron spectrum comes from the $[\pi / 2, \pi / 2]$ point. In this case, the wave vector $\mathbf{k}$ in $Z_{h F}(\mathbf{k})$ in Eq. (28) and $\Sigma_{1 e}^{(h)}(\mathbf{k})$ can be chosen as $Z_{h F}^{-1}=1-\left.\Sigma_{1 o}^{(h)}(\mathbf{k})\right|_{\mathbf{k}=[\pi / 2, \pi / 2]}$ and $\Sigma_{1 e}^{(h)}=$ $\left.\Sigma_{1 e}^{(h)}(\mathbf{k})\right|_{\mathbf{k}=[\pi / 2, \pi / 2]}$, then the equation satisfied by the dressed holon quasiparticle coherent weight $Z_{h F}$ in Eq. (33b) is reduced as,

$$
\begin{aligned}
\frac{1}{Z_{h F}} & =1+\frac{1}{N^{2}} \sum_{\mathbf{q}, \mathbf{p}} \Lambda^{2}\left(\mathbf{p}+\mathbf{k}_{N}\right) Z_{h F} \frac{B_{\mathbf{q}} B_{\mathbf{p}}}{4 \omega_{\mathbf{q}} \omega_{\mathbf{p}}} \\
& \times\left(\frac{F_{1}(\mathbf{q}, \mathbf{p})}{\left(\omega_{\mathbf{p}}-\omega_{\mathbf{q}}-\bar{\xi}_{\left.\mathbf{p}-\mathbf{q}+\mathbf{k}_{N}\right)^{2}}\right.}+\frac{F_{2}(\mathbf{q}, \mathbf{p})}{\left(\omega_{\mathbf{p}}-\omega_{\mathbf{q}}-\bar{\xi}_{\left.\mathbf{p}-\mathbf{q}+\mathbf{k}_{N}\right)^{2}}\right.}\right. \\
& \left.+\frac{F_{3}(\mathbf{q}, \mathbf{p})}{\left(\omega_{\mathbf{p}}+\omega_{\mathbf{q}}-\bar{\xi}_{\left.\mathbf{p}-\mathbf{q}+\mathbf{k}_{N}\right)^{2}}\right.}+\frac{F_{4}(\mathbf{q}, \mathbf{p})}{\left(\omega_{\mathbf{p}}+\omega_{\mathbf{q}}+\bar{\xi}_{\mathbf{p}-\mathbf{q}+\mathbf{k}_{N}}\right)^{2}}\right)
\end{aligned}
$$

where $\mathbf{k}_{N}=[\pi / 2, \pi / 2]$, and

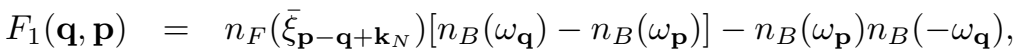

$$
\begin{aligned}
& F_{2}(\mathbf{q}, \mathbf{p})=n_{F}\left(\bar{\xi}_{\left.\mathbf{p}-\mathbf{q}+\mathbf{k}_{N}\right)}\right)\left[n_{B}\left(\omega_{\mathbf{p}}\right)-n_{B}\left(\omega_{\mathbf{q}}\right)\right]-n_{B}\left(\omega_{\mathbf{q}}\right) n_{B}\left(-\omega_{\mathbf{p}}\right), \\
& F_{3}(\mathbf{q}, \mathbf{p})=n_{F}\left(\bar{\xi}_{\left.\mathbf{p}-\mathbf{q}+\mathbf{k}_{N}\right)}\right)\left[n_{B}\left(\omega_{\mathbf{q}}\right)-n_{B}\left(-\omega_{\mathbf{p}}\right)\right]+n_{B}\left(\omega_{\mathbf{p}}\right) n_{B}\left(\omega_{\mathbf{q}}\right), \\
& \left.F_{4}(\mathbf{q}, \mathbf{p})=n_{F}\left(\bar{\xi}_{\mathbf{p}-\mathbf{q}+\mathbf{k}_{N}}\right) n_{B}\left(-\omega_{\mathbf{q}}\right)-n_{B}\left(\omega_{\mathbf{p}}\right)\right]+n_{B}\left(-\omega_{\mathbf{p}}\right) n_{B}\left(-\omega_{\mathbf{q}}\right) \text {. }
\end{aligned}
$$

As in the superconducting state, this self-consistent equation must be solved simultaneously with other self-consistent equations in Eqs (35), then the electron normal Green's function in Eq. (38a) and the electron spectral function in Eq. (39) in the normal state are reduced as,

$$
\begin{aligned}
G(\mathbf{k}, \omega) & =\frac{1}{N} \sum_{\mathbf{p}} Z_{h F} \frac{B_{\mathbf{p}}}{2 \omega_{\mathbf{p}}}\left(\frac{L_{1}(\mathbf{k}, \mathbf{p})}{\omega+\bar{\xi}_{\mathbf{p}+\mathbf{k}}-\omega_{\mathbf{p}}}+\frac{L_{2}(\mathbf{k}, \mathbf{p})}{\omega+\bar{\xi}_{\mathbf{p}+\mathbf{k}}+\omega_{\mathbf{p}}}\right) \\
A(\mathbf{k}, \omega) & =2 \pi \frac{1}{N} \sum_{\mathbf{p}} Z_{h F} \frac{B_{\mathbf{p}}}{2 \omega_{\mathbf{p}}}\left[L_{1}(\mathbf{k}, \mathbf{p}) \delta\left(\omega+\bar{\xi}_{\mathbf{p}+\mathbf{k}}-\omega_{\mathbf{p}}\right)\right. \\
& \left.+L_{2}(\mathbf{k}, \mathbf{p}) \delta\left(\omega+\bar{\xi}_{\mathbf{p}+\mathbf{k}}+\omega_{\mathbf{p}}\right)\right]
\end{aligned}
$$

where $L_{1}(\mathbf{k}, \mathbf{p})=n_{F}\left(\bar{\xi}_{\mathbf{p}+\mathbf{k}}\right)+n_{B}\left(\omega_{\mathbf{p}}\right)$ and $L_{2}(\mathbf{k}, \mathbf{p})=1-n_{F}\left(\bar{\xi}_{\mathbf{p}+\mathbf{k}}\right)+n_{B}\left(\omega_{\mathbf{p}}\right)$.

We have performed a calculation for the electron spectral function in Eq. $(47 \mathrm{~b})$ in the normal state, and the results at (a) the $[\pi, 0]$ point and (b) the $[\pi / 2, \pi / 2]$ point with $T=0.1 J$ for $t / J=2.5$ and $t^{\prime} / t=0.15$ at $\delta=0.09$ 

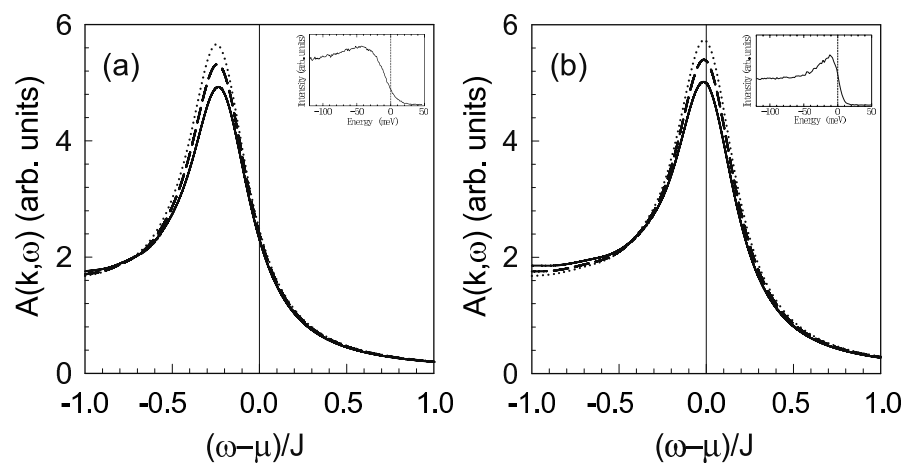

Figure 1.5: The electron spectral function $A(\mathbf{k}, \omega)$ at (a) the $[\pi, 0]$ point (b) the $[\pi / 2, \pi / 2]$ point in the normal state with $T=0.1 J$ in $\delta=0.09$ (solid line), $\delta=0.12$ (dashed line), and $\delta=0.15$ (dotted line) for $t / J=2.5$ and $t^{\prime} / t=0.15$. Inset: the corresponding experimental result of the single layer cuprate superconductor $\mathrm{Bi}_{2} \mathrm{Sr}_{2} \mathrm{CuO}_{6+\delta}$ at the $[\pi, 0]$ point and $[\pi / 2, \pi / 2]$ point, respectively, in the normal state taken from Ref. [73].

(solid line), $\delta=0.12$ (dashed line), and $\delta=0.15$ (dotted line) are plotted in Fig. 5 in comparison with the corresponding experimental result [73] of the single layer cuprate superconductor $\mathrm{Bi}_{2} \mathrm{Sr}_{2} \mathrm{CuO}_{6+\delta}$ in the normal state (inset). It is shown that (1) although both positions of the quasiparticle peaks at the $[\pi, 0]$ and $[\pi / 2, \pi / 2]$ points are below the Fermi energy, the position of the quasiparticle peak at the $[\pi / 2, \pi / 2]$ point is more close to the Fermi energy, which indicates that the lowest energy states are located at the $[\pi / 2, \pi / 2]$ point. In other words, the low energy spectral weight with the majority contribution to the low-energy properties of cuprate superconductors in the normal state comes from the $[\pi / 2, \pi / 2]$ point; (2) The electron spectrum in the normal state is doping dependence as in the superconducting state. The quasiparticle peaks at the $[\pi, 0]$ and $[\pi / 2, \pi / 2]$ points become sharper, while the spectral weight of these peaks increases in intensity with increasing doping. Furthermore, we have also discussed the temperature dependence of the electron spectrum in the normal state, and the results show that the spectral weight is suppressed with increasing temperatures. Our these results are qualitatively consistent with the ARPES experimental data of the single layer cuprate superconductors in the norma state [3, 10, 11, 12, 67, 73.

To elucidate the anomalous form of the electron spectrum $A(\mathbf{k}, \omega)$ in the normal state as a function of energy $\omega$ for $\mathbf{k}$ in the vicinity of the $[\pi, 0]$ point, we have discussed the electron spectral function around the $[\pi, 0]$ point, and the results with $T=0.1 J$ in $\delta=0.15$ for $t / J=2.5$ and $t^{\prime} / t=0.15$ at the $[0.9 \pi, 0]$ (solid line), $[0.95 \pi, 0]$ (long dashed line), $[\pi, 0]$ (short dashed line), $[\pi, 0.05 \pi]$ (dashdotted line), and $[\pi, 0.1 \pi]$ (dotted line) points are plotted in Fig. 6. Obviously, the positions of these peaks of the electron spectral function $A(\mathbf{k}, \omega)$ around 


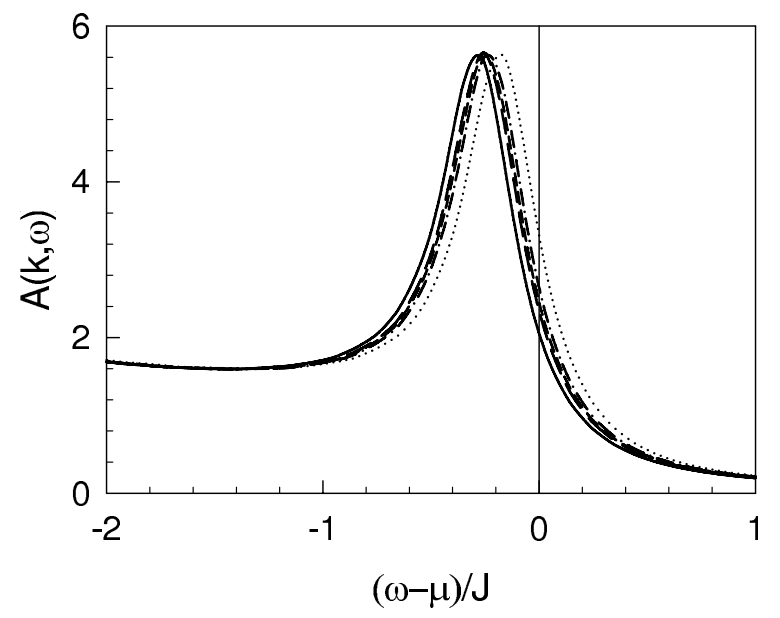

Figure 1.6: The electron spectral function $A(\mathbf{k}, \omega)$ in the normal state with $T=0.1 J$ in $\delta=0.15$ at the $[0.9 \pi, 0]$ (solid line), [0.95, 0$]$ (long dashed line), $[\pi, 0]$ (short dashed line), $[\pi, 0.05 \pi]$ (dash-dotted line), and $[\pi, 0.1 \pi]$ (dotted line) points for $t / J=2.5$ and $t^{\prime} / t=0.15$.

the $[\pi, 0]$ point in the normal state are almost not changeable, which leads to the unusual quasiparticle dispersion around the $[\pi, 0]$ point. In particular, the lowest energy peaks in the normal state are well defined at all momenta. To show this broad feature in the electron spectrum around the $[\pi, 0]$ point clearly, we plot the positions of the lowest energy quasiparticle peaks in the electron spectral function as a function of momentum along the high symmetry directions with $T=0.1 J$ at $\delta=0.15$ for $t / J=2.5$ and $t^{\prime} / t=0.15$ in Fig. 7. For comparison, the corresponding result of the bare electron dispersion of the $t-t^{\prime}$ model (dotted line), and experimental result (inset) of the electron dispersion from the single layer cuprate superconductor $\mathrm{Bi}_{2} \mathrm{Sr}_{2} \mathrm{CuO}_{6+\delta}$ in the normal state 3 are also shown in Fig. 7. In accordance with the anomalous property of the electron spectrum in Fig. 6, the electron quasiparticles around the $[\pi, 0]$ point disperse very weakly with momentum, and then the unusual flat band appears, while the Fermi energy is only slightly above this flat band, in qualitative agreement with these obtained from ARPES experimental measurements on the single layer cuprate superconductors in the normal state [3, 10, 11, 12, 67, 73.

Since the full electron Green's function (then the electron spectral function) in the normal state is obtained beyond the mean-field approximation by considering the fluctuation due to the spin pair bubble, therefore the nature of the electron spectrum in the normal state is closely related to the strong coupling between the dressed holon (then electron quasiparticles) and collective magnetic excitations. This can be understood from a comparison between the bare electron dispersion of the $t-t^{\prime}$ model and renormalized electron quasiparticle dispersion of the $t-J$ model in Fig. 7. Our results show that the single-particle 


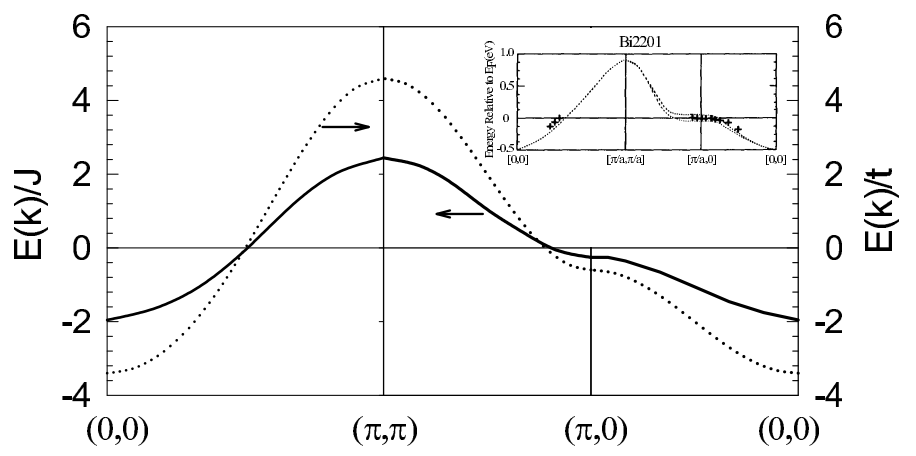

Figure 1.7: The position of the lowest energy quasiparticle peaks in $A(\mathbf{k}, \omega)$ in the normal state as a function of momentum for $t / J=2.5$ and $t^{\prime} / t=0.15$ with $T=0.1 J$ at $\delta=0.15$. The dotted line is corresponding result of the bare electron dispersion of the $t-t^{\prime}$ model. Inset: the corresponding experimental result of the single layer cuprate superconductor $\mathrm{Bi}_{2} \mathrm{Sr}_{2} \mathrm{CuO}_{6+\delta}$ in the normal state taken from Ref. [3].

hopping in the $t-J$ model is strongly renormalized by the magnetic interaction. As a consequence, the quasiparticle bandwidth is reduced to the order of (a few) $J$, and therefore the energy scale of the quasiparticle band is controlled by the magnetic interaction. This renormalization due to the strong interaction is then responsible for the unusual electron quasiparticle spectrum and production of the flat band. Moreover, these results also show that the electron quasiparticle excitations originating from the dressed holons and spins are due to the charge-spin recombination, this reflects the composite nature of the electron quasiparticle excitations, and then the unconventional normal state properties in cuprate superconductors are attributed to the presence of the dressed holon, spin, and electron quasiparticle excitations.

\subsection{Electronic structure of the bilayer cuprate superconductors}

Recently, the improvements in the resolution of the ARPES experiments 4, 5, 74, 75, 76] allowed to resolve additional features in the electron spectral function $A(\mathbf{k}, \omega)$. Among these new achievements is the observation of the bilayer splitting in the bilayer cuprate superconductors in both normal and superconducting states [4, 74, 75, 76. In this case, whether the electronic structure of cuprate superconductors can be influenced by the interaction between $\mathrm{CuO}_{2}$ planes has been an interesting issue. The study of the electronic structure is complicated by the bilayer splitting, that is, the bilayer splitting of the $\mathrm{CuO}_{2}$ planes derived the electronic structure in the bonding and antibonding bands due to the present of $\mathrm{CuO}_{2}$ bilayer blocks in the unit cell [74, 75, 76]. The magnitude of the 
bilayer splitting is the doping independent, and increases upon approaching the $[\pi, 0]$ point, where the bilayer splitting exhibits the largest value. As a result of the maximal bilayer splitting at the $[\pi, 0]$ point, there are two main flat bands around the $[\pi, 0]$ point in the normal state $[74,75,76]$. In corresponding to this double-peak structure in the normal state, the peak-dip-hump structure around the $[\pi, 0]$ point in the superconducting state is observed [4, 20, 21, 22, 23, 24] as mentioned in section 1. Therefore an important issue is whether the behavior of the low energy excitations determined by the electronic structure of cuprate superconductors is universal or not. The earlier works [64, 4] gave the main impetus for a phenomenological description of the single-particle excitations in terms of an interaction between quasiparticles and collective modes, which is of fundamental relevance to the nature of superconductivity and the pairing mechanism in cuprate superconductors. However, the different interpretive scenario has been proposed 75, 4, where it has been argued that the main features of the peak-dip-hump structure is caused by the bilayer splitting [75, 74, 76, 77,, with the peak and hump corresponding to the antibonding and bonding bands, respectively. Furthermore, some ARPES experimental data measured above and below the superconducting transition temperature show that this peak-diphump structure is totally unrelated to superconductivity [74. In particular, the recent ARPES experimental results reported by several groups support this scenario, and most convincingly suggested that the peak-dip-hump structure originates from the bilayer splitting at any doping levels [25. In this section we show explicitly if the bilayer interaction is included in the framework of the kinetic energy driven superconductivity, one can reproduce some main features in both normal and superconducting states observed experimentally on the bilayer cuprate superconductors $4,74,75,76$.

\subsubsection{Electronic structure of the bilayer cuprate supercon- ductors in the superconducting state}

For discussions of the electronic structure of the bilayer cuprate superconductors, the $t$ - $J$ model in Eq. (1) can be generalized by including the bilayer hopping and bilayer magnetic exchange interaction as,

$$
\begin{aligned}
H & =-t \sum_{i \hat{\eta} a \sigma} C_{i a \sigma}^{\dagger} C_{i+\hat{\eta} a \sigma}+t^{\prime} \sum_{i \hat{\tau} a \sigma} C_{i a \sigma}^{\dagger} C_{i+\hat{\tau} a \sigma}-\sum_{i \sigma} t_{\perp}(i)\left(C_{i 1 \sigma}^{\dagger} C_{i 2 \sigma}+H . c .\right) \\
& +\mu_{0} \sum_{i a \sigma} C_{i a \sigma}^{\dagger} C_{i a \sigma}+J \sum_{i \hat{\eta} a} \mathbf{S}_{i a} \cdot \mathbf{S}_{i+\hat{\eta} a}+J_{\perp} \sum_{i} \mathbf{S}_{i 1} \cdot \mathbf{S}_{i 2}
\end{aligned}
$$

where $a=1,2$ is plane index, and the interlayer hopping [78],

$$
t_{\perp}(\mathbf{k})=\frac{t_{\perp}}{4}\left(\cos k_{x}-\cos k_{y}\right)^{2}
$$

describes coherent hopping between the $\mathrm{CuO}_{2}$ planes. This functional form of the interlayer hopping in Eq. (49) is firstly predicted on the basis of the local density approximation calculations [78, and later the experimental observed 
bilayer spilitting agrees well with it 4, 74, 75, 76. In the charge-spin separation fermion-spin representation (9), this bilayer $t-J$ model (48) can be expressed as,

$$
\begin{aligned}
H & =t \sum_{i \hat{\eta} a}\left(h_{i+\hat{\eta} a \uparrow}^{\dagger} h_{i a \uparrow} S_{i a}^{+} S_{i+\hat{\eta} a}^{-}+h_{i+\hat{\eta} a \downarrow}^{\dagger} h_{i a \downarrow} S_{i a}^{-} S_{i+\hat{\eta} a}^{+}\right) \\
& -t^{\prime} \sum_{i \hat{\tau} a}\left(h_{i+\hat{\tau} a \uparrow}^{\dagger} h_{i a \uparrow} S_{i a}^{+} S_{i+\hat{\tau} a}^{-}+h_{i+\hat{\tau} a \downarrow}^{\dagger} h_{i a \downarrow} S_{i a}^{-} S_{i+\hat{\tau} a}^{+}\right) \\
& +\sum_{i} t_{\perp}(i)\left(h_{i 2 \uparrow}^{\dagger} h_{i 1 \uparrow} S_{i 1}^{+} S_{i 2}^{-}+h_{i 1 \uparrow}^{\dagger} h_{i 2 \uparrow} S_{i 2}^{+} S_{i 1}^{-}\right. \\
& \left.+h_{i 2 \downarrow}^{\dagger} h_{i 1 \downarrow} S_{i 1}^{-} S_{i 2}^{+}+h_{i 1 \downarrow}^{\dagger} h_{i 2 \downarrow} S_{i 2}^{-} S_{i 1}^{+}\right)-\mu_{0} \sum_{i a \sigma} h_{i a \sigma}^{\dagger} h_{i a \sigma} \\
& +J_{\mathrm{eff}} \sum_{i \hat{\eta} a} \mathbf{S}_{i a} \cdot \mathbf{S}_{i+\hat{\eta} a}+J_{\mathrm{eff} \perp} \sum_{i} \mathbf{S}_{i 1} \cdot \mathbf{S}_{i 2},
\end{aligned}
$$

where $J_{\text {eff } \perp}=J_{\perp}(1-\delta)^{2}$. Since there are two coupled $\mathrm{CuO}_{2}$ planes in the unit cell, therefore the superconducting order parameter for the electron Cooper pair is a matrix $\Delta=\Delta_{L}+\sigma_{x} \Delta_{T}$, with the longitudinal and transverse superconducting order parameters can be expressed as [37,

$$
\begin{aligned}
\Delta_{L} & =\left\langle C_{i a \uparrow}^{\dagger} C_{i+\hat{\eta} a \downarrow}^{\dagger}-C_{i a \downarrow}^{\dagger} C_{i+\hat{\eta} a \uparrow}^{\dagger}\right\rangle \\
& =\left\langle h_{i a \uparrow} h_{i+\hat{\eta} a \downarrow} S_{i a}^{+} S_{i+\hat{\eta} a}^{-}-h_{i a \downarrow} h_{i+\hat{\eta} a \uparrow} S_{i a}^{-} S_{i+\hat{\eta} a}^{+}\right\rangle=-\chi_{1} \Delta_{h L}, \\
\Delta_{T} & =\left\langle C_{i 1 \uparrow}^{\dagger} C_{i 2 \downarrow}^{\dagger}-C_{i 1 \downarrow}^{\dagger} C_{i 2 \uparrow}^{\dagger}\right\rangle \\
& =\left\langle h_{i 1 \uparrow} h_{i 2 \downarrow} S_{i 1}^{+} S_{i 2}^{-}-h_{i 1 \downarrow} h_{i 2 \uparrow} S_{i 1}^{-} S_{i 2}^{+}\right\rangle=-\chi_{\perp} \Delta_{h T},
\end{aligned}
$$

respectively, where the spin correlation functions $\chi_{1}=\left\langle S_{i a}^{+} S_{i+\hat{\eta} a}^{-}\right\rangle$and $\chi_{\perp}=$ $\left\langle S_{i 1}^{+} S_{i 2}^{-}\right\rangle$, and the longitudinal and transverse dressed holon pairing order parameters,

$$
\begin{aligned}
\Delta_{h L} & =\left\langle h_{i+\hat{\eta} a \downarrow} h_{i a \uparrow}-h_{i+\hat{\eta} a \uparrow} h_{i a \downarrow}\right\rangle \\
\Delta_{h T} & =\left\langle h_{i 2 \downarrow} h_{i 1 \uparrow}-h_{i 2 \uparrow} h_{i 1 \downarrow}\right\rangle .
\end{aligned}
$$

In the following discussions, our main goal is to generalize the analytical calculation from the single layer case [35] in section 3 to the bilayer system 37. As in the case for the superconducting order parameter, the full dressed holon normal and anomalous Green's functions also can be expressed as matrices $g(\mathbf{k}, \omega)=g_{L}(\mathbf{k}, \omega)+\sigma_{x} g_{T}(\mathbf{k}, \omega)$ and $\Im^{\dagger}(\mathbf{k}, \omega)=\Im_{L}^{\dagger}(\mathbf{k}, \omega)+\sigma_{x} \Im_{L}^{\dagger}(\mathbf{k}, \omega)$, respectively. We now can follow the discussions for the single layer case in section 3 35, 32, and evaluate explicitly these corresponding longitudinal and transverse parts of the full dressed holon normal and anomalous Green's functions as,

$$
\begin{aligned}
g_{L}(\mathbf{k}, \omega) & =\frac{1}{2} \sum_{\nu=1,2} Z_{h F A}^{(\nu)}\left(\frac{U_{h \nu \mathbf{k}}^{2}}{\omega-E_{h \nu \mathbf{k}}}+\frac{V_{h \nu \mathbf{k}}^{2}}{\omega+E_{h \nu \mathbf{k}}}\right) \\
g_{T}(\mathbf{k}, \omega) & =\frac{1}{2} \sum_{\nu=1,2}(-1)^{\nu+1} Z_{h F A}^{(\nu)}\left(\frac{U_{h \nu \mathbf{k}}^{2}}{\omega-E_{h \nu \mathbf{k}}}+\frac{V_{h \nu \mathbf{k}}^{2}}{\omega+E_{h \nu \mathbf{k}}}\right),
\end{aligned}
$$




$$
\begin{aligned}
& \Im_{L}^{\dagger}(\mathbf{k}, \omega)=-\frac{1}{2} \sum_{\nu=1,2} Z_{h F A}^{(\nu)} \frac{\bar{\Delta}_{h z}^{(\nu)}(\mathbf{k})}{2 E_{h \nu \mathbf{k}}}\left(\frac{1}{\omega-E_{h \nu \mathbf{k}}}-\frac{1}{\omega+E_{h \nu \mathbf{k}}}\right), \\
& \Im_{T}^{\dagger}(\mathbf{k}, \omega)=-\frac{1}{2} \sum_{\nu=1,2}(-1)^{\nu+1} Z_{h F A}^{(\nu)} \frac{\bar{\Delta}_{h z}^{(\nu)}(\mathbf{k})}{2 E_{h \nu \mathbf{k}}}\left(\frac{1}{\omega-E_{h \nu \mathbf{k}}}-\frac{1}{\omega+E_{h \nu \mathbf{k}}}\right),
\end{aligned}
$$

with the dressed holon quasiparticle coherence factors,

$$
\begin{aligned}
U_{h \nu \mathbf{k}}^{2} & =\frac{1}{2}\left(1+\frac{\bar{\xi}_{\nu \mathbf{k}}}{E_{h \nu \mathbf{k}}}\right), \\
V_{h \nu \mathbf{k}}^{2} & =\frac{1}{2}\left(1-\frac{\bar{\xi}_{\nu \mathbf{k}}}{E_{h \nu \mathbf{k}}}\right),
\end{aligned}
$$

and

$$
\begin{aligned}
E_{h \nu \mathbf{k}} & =\sqrt{\left[\bar{\xi}_{\nu \mathbf{k}}\right]^{2}+\left|\bar{\Delta}_{h z}^{(\nu)}(\mathbf{k})\right|^{2}}, \\
\bar{\xi}_{\nu \mathbf{k}} & =Z_{h F A}^{(\nu)} \epsilon_{\nu \mathbf{k}}-\mu, \\
\bar{\Delta}_{h z}^{(\nu)}(\mathbf{k}) & =Z_{h F A}^{(\nu)}\left[\bar{\Delta}_{h L}(\mathbf{k})+(-1)^{\nu+1} \bar{\Delta}_{h T}(\mathbf{k})\right], \\
\mu & =Z_{h F A}^{(\nu)}\left(\mu_{0}-\Sigma_{1 L e}^{(h)}\right),
\end{aligned}
$$

are the dressed holon quasiparticle spectrum, the renormalized dressed holon excitation spectrum, the renormalized dressed holon pair gap function, and renormalized chemical potential, respectively, where $\epsilon_{\nu \mathbf{k}}=Z t \chi_{1} \gamma_{\mathbf{k}}-Z t^{\prime} \chi_{2} \gamma^{\prime}{ }_{\mathbf{k}}+$ $(-1)^{\nu+1} \chi_{\perp} t_{\perp}(\mathbf{k})$, the spin correlation function $\chi_{2}=\left\langle S_{i a}^{+} S_{i+\hat{\tau} a}^{-}\right\rangle$, the longitudinal and transverse effective dressed holon pair gap functions, $\bar{\Delta}_{h L}(\mathbf{k})=$ $\left.\Sigma_{2 L}^{(h)}(\mathbf{k}, \omega)\right|_{\omega=0}=\bar{\Delta}_{h L} \gamma_{\mathbf{k}}^{(d)}, \bar{\Delta}_{h T}(\mathbf{k})=\left.\Sigma_{2 T}^{(h)}(\mathbf{k}, \omega)\right|_{\omega=0}=\bar{\Delta}_{h T}$, and the dressed holon quasiparticle coherent weights,

$$
\begin{aligned}
& \frac{1}{Z_{h F A}^{(1)}}=\frac{1}{Z_{h F 1}}-\frac{1}{Z_{h F 2}}, \\
& \frac{1}{Z_{h F A}^{(2)}}=\frac{1}{Z_{h F 1}}+\frac{1}{Z_{h F 2}},
\end{aligned}
$$

with the longitudinal and transverse dressed holon quasiparticle coherent weights,

$$
\begin{aligned}
& \frac{1}{Z_{h F 1}}=1-\left.\Sigma_{1 L}^{(h o)}\left(\mathbf{k}_{A}, \omega\right)\right|_{\omega=0}, \\
& \frac{1}{Z_{h F 2}}=\left.\Sigma_{1 T}^{(h o)}\left(\mathbf{k}_{A}, \omega\right)\right|_{\omega=0},
\end{aligned}
$$

where $\Sigma_{1 L}^{(h o)}(\mathbf{k}, \omega)$ and $\Sigma_{1 T}^{(h o)}(\mathbf{k}, \omega)$ are the corresponding antisymmetric parts of the longitudinal and transverse dressed holon self-energy functions $\Sigma_{1 L}^{(h)}(\mathbf{k}, \omega)$ 
and $\Sigma_{1 T}^{(h)}(\mathbf{k}, \omega)$, while the longitudinal and transverse parts of the dressed holon self-energy functions $\Sigma_{1}^{(h)}(\mathbf{k}, \omega)$ and $\Sigma_{2}^{(h)}(\mathbf{k}, \omega)$ are given by [37],

$$
\begin{aligned}
\Sigma_{1 L}^{(h)}\left(\mathbf{k}, i \omega_{n}\right) & =\frac{1}{N^{2}} \sum_{\mathbf{p}, \mathbf{q}}\left[R_{\mathbf{p}+\mathbf{q}+\mathbf{k}}^{(1)} \frac{1}{\beta} \sum_{i p_{m}} g_{L}\left(\mathbf{p}+\mathbf{k}, i p_{m}+i \omega_{n}\right) \Pi_{L L}\left(\mathbf{p}, \mathbf{q}, i p_{m}\right)\right. \\
& \left.+R_{\mathbf{p}+\mathbf{q}+\mathbf{k}}^{(2)} \frac{1}{\beta} \sum_{i p_{m}} g_{T}\left(\mathbf{p}+\mathbf{k}, i p_{m}+i \omega_{n}\right) \Pi_{T L}\left(\mathbf{p}, \mathbf{q}, i p_{m}\right)\right], \quad(1.58 \mathrm{a}) \\
\Sigma_{1 T}^{(h)}\left(\mathbf{k}, i \omega_{n}\right) & =\frac{1}{N^{2}} \sum_{\mathbf{p}, \mathbf{q}}\left[R_{\mathbf{p}+\mathbf{q}+\mathbf{k}}^{(1)} \frac{1}{\beta} \sum_{i p_{m}} g_{T}\left(\mathbf{p}+\mathbf{k}, i p_{m}+i \omega_{n}\right) \Pi_{T T}\left(\mathbf{p}, \mathbf{q}, i p_{m}\right)\right. \\
& \left.+R_{\mathbf{p}+\mathbf{q}+\mathbf{k}}^{(2)} \frac{1}{\beta} \sum_{i p_{m}} g_{L}\left(\mathbf{p}+\mathbf{k}, i p_{m}+i \omega_{n}\right) \Pi_{L T}\left(\mathbf{p}, \mathbf{q}, i p_{m}\right)\right], \quad(1.58 \mathrm{~b}) \\
\Sigma_{2 L}^{(h)}\left(\mathbf{k}, i \omega_{n}\right) & =\frac{1}{N^{2}} \sum_{\mathbf{p}, \mathbf{q}}\left[R_{\mathbf{p}+\mathbf{q}+\mathbf{k}}^{(1)} \frac{1}{\beta} \sum_{i p_{m}} \Im_{L}^{\dagger}\left(\mathbf{p}+\mathbf{k}, i p_{m}+i \omega_{n}\right) \Pi_{L L}\left(\mathbf{p}, \mathbf{q}, i p_{m}\right)\right. \\
& \left.+R_{\mathbf{p}+\mathbf{q}+\mathbf{k}}^{(2)} \frac{1}{\beta} \sum_{i p_{m}} \Im_{T}^{\dagger}\left(\mathbf{p}+\mathbf{k}, i p_{m}+i \omega_{n}\right) \Pi_{T L}\left(\mathbf{p}, \mathbf{q}, i p_{m}\right)\right], \quad(1.58 \mathrm{c}) \\
\Sigma_{2 T}^{(h)}\left(\mathbf{k}, i \omega_{n}\right) & =\frac{1}{N^{2}} \sum_{\mathbf{p}, \mathbf{q}}\left[R_{\mathbf{p}+\mathbf{q}+\mathbf{k}}^{(1)} \frac{1}{\beta} \sum_{i p_{m}} \Im_{T}^{\dagger}\left(\mathbf{p}+\mathbf{k}, i p_{m}+i \omega_{n}\right) \Pi_{T T}\left(\mathbf{p}, \mathbf{q}, i p_{m}\right)\right. \\
& \left.+R_{\mathbf{p}+\mathbf{q}+\mathbf{k}}^{(2)} \frac{1}{\beta} \sum_{i p_{m}} \Im_{L}^{\dagger}\left(\mathbf{p}+\mathbf{k}, i p_{m}+i \omega_{n}\right) \Pi_{L T}\left(\mathbf{p}, \mathbf{q}, i p_{m}\right)\right], \quad(1.58 \mathrm{~d})
\end{aligned}
$$

where $R_{\mathbf{k}}^{(1)}=\left[Z\left(t \gamma_{\mathbf{k}}-t^{\prime} \gamma_{\mathbf{k}}^{\prime}\right)\right]^{2}+t_{\perp}^{2}(\mathbf{k}), R_{\mathbf{k}}^{(2)}=2 Z\left(t \gamma_{\mathbf{k}}-t^{\prime} \gamma_{\mathbf{k}}^{\prime}\right) t_{\perp}(\mathbf{k})$, and the spin bubbles,

$$
\Pi_{\eta, \eta^{\prime}}\left(\mathbf{p}, \mathbf{q}, i p_{m}\right)=\frac{1}{\beta} \sum_{i q_{m}} D_{\eta}^{(0)}\left(\mathbf{q}, i q_{m}\right) D_{\eta^{\prime}}^{(0)}\left(\mathbf{q}+\mathbf{p}, i q_{m}+i p_{m}\right),
$$

with $\eta=L, T$ and $\eta^{\prime}=L, T$, and the mean-field spin Green's function $D^{(0)}(\mathbf{k}, \omega)=$ $D_{L}^{(0)}(\mathbf{k}, \omega)+\sigma_{x} D_{T}^{(0)}(\mathbf{k}, \omega)$, with the corresponding longitudinal and transverse parts have been obtained as [37,

$$
\begin{aligned}
D_{L}^{(0)}(\mathbf{k}, \omega) & =\frac{1}{2} \sum_{\nu=1,2} \frac{B_{\nu \mathbf{k}}}{\omega^{2}-\omega_{\nu \mathbf{k}}^{2}} \\
D_{T}^{(0)}(\mathbf{k}, \omega) & =\frac{1}{2} \sum_{\nu=1,2}(-1)^{\nu+1} \frac{B_{\nu \mathbf{k}}}{\omega^{2}-\omega_{\nu \mathbf{k}}^{2}}
\end{aligned}
$$

where $B_{\nu \mathbf{k}}=\lambda\left(A_{1} \gamma_{\mathbf{k}}-A_{2}\right)-\lambda^{\prime}\left(2 \chi_{2}^{z} \gamma^{\prime} \mathbf{k}-\chi_{2}\right)-J_{\mathrm{eff} \perp}\left[\chi_{\perp}+2 \chi_{\perp}^{z}(-1)^{\nu}\right]\left[\epsilon_{\perp}(\mathbf{k})+\right.$ $\left.(-1)^{\nu}\right], A_{1}=2 \epsilon_{\|} \chi_{1}^{z}+\chi_{1}, A_{2}=\epsilon_{\|} \chi_{1}+2 \chi_{1}^{z}, \lambda=2 Z J_{\text {eff }}, \lambda^{\prime}=4 Z \phi_{2} t^{\prime}, \epsilon_{\|}=$ $1+2 t \phi_{1} / J_{\text {eff }}, \epsilon_{\perp}(\mathbf{k})=1+4 \phi_{\perp} t_{\perp}(\mathbf{k}) / J_{\text {eff } \perp}$, the spin correlation functions $\chi_{1}^{z}=$ $\left\langle S_{i a}^{z} S_{i+\hat{\eta} a}^{z}\right\rangle, \chi_{2}^{z}=\left\langle S_{i a}^{z} S_{i+\hat{\tau} a}^{z}\right\rangle, \chi_{\perp}^{z}=\left\langle S_{i 1}^{z} S_{i 2}^{z}\right\rangle$, the dressed holon particle-hole order parameters $\phi_{1}=\left\langle h_{i a \sigma}^{\dagger} h_{i+\hat{\eta} a \sigma}\right\rangle, \phi_{2}=\left\langle h_{i a \sigma}^{\dagger} h_{i+\hat{\tau} a \sigma}\right\rangle, \phi_{\perp}=\left\langle h_{i 1 \sigma}^{\dagger} h_{i 2 \sigma}\right\rangle$, and 
the mean-field spin excitation spectrum,

$$
\begin{aligned}
\omega_{\nu \mathbf{k}}^{2} & =\lambda^{2}\left[\left(A_{4}-\alpha \epsilon_{\|} \chi_{1}^{z} \gamma_{\mathbf{k}}-\frac{1}{2 Z} \alpha \epsilon_{\|} \chi_{1}\right)\left(1-\epsilon_{\|} \gamma_{\mathbf{k}}\right)\right. \\
& \left.+\frac{1}{2} \epsilon_{\|}\left(A_{3}-\frac{2}{Z} \alpha \chi_{1}^{z}-\alpha \chi_{1} \gamma_{\mathbf{k}}\right)\left(\epsilon_{\|}-\gamma_{\mathbf{k}}\right)\right] \\
& +\lambda^{\prime 2}\left[\alpha\left(\chi_{2}^{z} \gamma_{\mathbf{k}}^{\prime}-\frac{Z-1}{2 Z} \chi_{2}\right) \gamma_{\mathbf{k}}^{\prime}+\frac{1}{2}\left(A_{5}-\frac{2}{Z} \alpha \chi_{2}^{z}\right)\right] \\
& +\lambda \lambda^{\prime} \alpha\left[\chi_{1}^{z}\left(1-\epsilon_{\|} \gamma_{\mathbf{k}}\right) \gamma_{\mathbf{k}}^{\prime}+\frac{1}{2}\left(\chi_{1} \gamma_{\mathbf{k}}^{\prime}-C_{2}\right)\left(\epsilon_{\|}-\gamma_{\mathbf{k}}\right)+\gamma_{\mathbf{k}}^{\prime}\left(C_{2}^{z}-\epsilon_{\|} \chi_{2}^{z} \gamma_{\mathbf{k}}\right)\right. \\
& \left.-\frac{1}{2} \epsilon_{\|}\left(C_{2}-\chi_{2} \gamma_{\mathbf{k}}\right)\right]+\lambda J_{\mathrm{eff} \perp} \alpha\left\{\frac{1}{2} \epsilon_{\perp}(\mathbf{k})\left(\epsilon_{\|}-\gamma_{\mathbf{k}}\right)\left[C_{\perp}+\chi_{1}(-1)^{\nu}\right]\right. \\
& +\left(1-\epsilon_{\|} \gamma_{\mathbf{k}}\right)\left[C_{\perp}^{z}+\chi_{1}^{z} \epsilon_{\perp}(\mathbf{k})(-1)^{\nu}\right] \\
& \left.+\left[\epsilon_{\perp}(\mathbf{k})+(-1)^{\nu}\right]\left[\frac{1}{2} \epsilon_{\|}\left(C_{\perp}-\chi_{\perp} \gamma_{\mathbf{k}}\right)+\left(C_{\perp}^{z}-\epsilon_{\|} \chi_{\perp}^{z} \gamma_{\mathbf{k}}\right)(-1)^{\nu}\right]\right\} \\
& +\lambda^{\prime} J_{\mathrm{eff} \perp} \alpha\left\{\gamma_{\mathbf{k}}^{\prime}\left[C_{\perp}^{\prime z}+\chi_{2}^{z} \epsilon_{\perp}(\mathbf{k})(-1)^{\nu}\right]-\frac{1}{2} \epsilon_{\perp}(\mathbf{k})\left[C_{\perp}^{\prime}+\chi_{2}(-1)^{\nu}\right]\right. \\
& \left.+\left[\frac{1}{2}\left(\chi_{\perp} \gamma_{\mathbf{k}}^{\prime}-C_{\perp}^{\prime}\right)+\chi_{\perp}^{z} \gamma_{\mathbf{k}}^{\prime}(-1)^{\nu}\right]\left[\epsilon_{\perp}(\mathbf{k})+(-1)^{\nu}\right]\right\} \\
& +\frac{1}{4} J_{\mathrm{eff} \perp}^{2}\left[\epsilon_{\perp}(\mathbf{k})+(-1)^{\nu}\right]^{2},
\end{aligned}
$$

where $A_{3}=\alpha C_{1}+(1-\alpha) / 2 Z, A_{4}=\alpha C_{1}^{z}+(1-\alpha) / 4 Z, A_{5}=\alpha C_{3}+(1-$ $\alpha) / 2 Z$, and the spin correlation functions $C_{1}=\left(1 / Z^{2}\right) \sum_{\hat{\eta} \hat{\eta}^{\prime}}\left\langle S_{i+\hat{\eta} a}^{+} S_{i+\hat{\eta}^{\prime} a}^{-}\right\rangle, C_{2}=$ $\left(1 / Z^{2}\right) \sum_{\hat{\eta} \hat{\tau}}\left\langle S_{i+\hat{\eta} a}^{+} S_{i+\hat{\tau} a}^{-}\right\rangle, C_{3}=\left(1 / Z^{2}\right) \sum_{\hat{\tau} \hat{\tau}^{\prime}}\left\langle S_{i+\hat{\tau} a}^{+} S_{i+\hat{\tau}^{\prime} a}^{-}\right\rangle, C_{1}^{z}=\left(1 / Z^{2}\right) \sum_{\hat{\eta} \hat{\eta}^{\prime}}$ $\left\langle S_{i+\hat{\eta} a}^{z} S_{i+\hat{\eta}^{\prime} a}^{z}\right\rangle, C_{2}^{z}=\left(1 / Z^{2}\right) \sum_{\hat{\eta} \hat{\tau}}\left\langle S_{i+\hat{\eta} a}^{z} S_{i+\hat{\tau} a}^{z}\right\rangle, C_{\perp}=(1 / Z) \sum_{\hat{\eta}}\left\langle S_{i 1}^{+} S_{i+\hat{\eta} 2}^{-}\right\rangle, C_{\perp}^{\prime}=$ $(1 / Z) \sum_{\hat{\tau}}\left\langle S_{i 1}^{+} S_{i+\hat{\tau} 2}^{-}\right\rangle, C_{\perp}^{z}=(1 / Z) \sum_{\hat{\eta}}\left\langle S_{i 1}^{z} S_{i+\hat{\eta} 2}^{z}\right\rangle, C_{\perp}^{\prime z}=(1 / Z) \sum_{\hat{\tau}}\left\langle S_{i 1}^{z} S_{i+\hat{\tau} 2}^{z}\right\rangle$.

As in the single layer case [35, 32] discussed in section 3 , we now can calculate the electron normal and anomalous Green's functions $G\left(i-j, t-t^{\prime}\right)=$ $\left\langle\left\langle C_{i \sigma}(t) ; C_{j \sigma}^{\dagger}\left(t^{\prime}\right)\right\rangle\right\rangle=G_{L}\left(i-j, t-t^{\prime}\right)+\sigma_{x} G_{T}\left(i-j, t-t^{\prime}\right)$ and $\Gamma^{\dagger}\left(i-j, t-t^{\prime}\right)=$ $\left\langle\left\langle C_{i \uparrow}^{\dagger}(t) ; C_{j \downarrow}^{\dagger}\left(t^{\prime}\right)\right\rangle\right\rangle=\Gamma_{L}^{\dagger}\left(i-j, t-t^{\prime}\right)+\sigma_{x} \Gamma_{T}^{\dagger}\left(i-j, t-t^{\prime}\right)$ in terms of the full dressed holon normal and anomalous Green's functions in Eqs. (53) and mean-field spin Green's function in Eqs. (60), and can be evaluated explicitly as,

$$
\begin{aligned}
G_{L}(\mathbf{k}, \omega) & =\frac{1}{8 N} \sum_{\mathbf{p}} \sum_{\mu \nu} Z_{h F A}^{(\mu)} \frac{B_{\nu \mathbf{p}}}{\omega_{\nu \mathbf{p}}}\left[L _ { \mu \nu } ^ { ( 1 ) } ( \mathbf { k } , \mathbf { p } ) \left(\frac{U_{h \mu \mathbf{p}-\mathbf{k}}^{2}}{\omega+E_{h \mu \mathbf{p}-\mathbf{k}}-\omega_{\nu \mathbf{p}}}\right.\right. \\
& \left.+\frac{V_{h \mu \mathbf{p}-\mathbf{k}}^{2}}{\omega-E_{h \mu \mathbf{p}-\mathbf{k}}+\omega_{\nu \mathbf{p}}}\right)+L_{\mu \nu}^{(2)}(\mathbf{k}, \mathbf{p})\left(\frac{U_{h \mu \mathbf{p}-\mathbf{k}}^{2}}{\omega+E_{h \mu \mathbf{p}-\mathbf{k}}+\omega_{\nu \mathbf{p}}}\right. \\
& \left.\left.+\frac{V_{h \mu \mathbf{p}-\mathbf{k}}^{2}}{\omega-E_{h \mu \mathbf{p}-\mathbf{k}}-\omega_{\nu \mathbf{p}}}\right)\right]
\end{aligned}
$$




$$
\begin{aligned}
G_{T}(\mathbf{k}, \omega) & =\frac{1}{8 N} \sum_{\mathbf{p}} \sum_{\mu \nu}(-1)^{\mu+\nu} Z_{h F A}^{(\mu)} \frac{B_{\nu \mathbf{p}}}{\omega_{\nu \mathbf{p}}}\left[L _ { \mu \nu } ^ { ( 1 ) } ( \mathbf { k } , \mathbf { p } ) \left(\frac{U_{h \mu \mathbf{p}-\mathbf{k}}^{2}}{\omega+E_{h \mu \mathbf{p}-\mathbf{k}}-\omega_{\nu \mathbf{p}}}\right.\right. \\
& \left.+\frac{V_{h \mu \mathbf{p}-\mathbf{k}}^{2}}{\omega-E_{h \mu \mathbf{p}-\mathbf{k}}+\omega_{\nu \mathbf{p}}}\right)+L_{\mu \nu}^{(2)}(\mathbf{k}, \mathbf{p})\left(\frac{U_{h \mu \mathbf{p}-\mathbf{k}}^{2}}{\omega+E_{h \mu \mathbf{p}-\mathbf{k}}+\omega_{\nu \mathbf{p}}}\right. \\
& \left.\left.+\frac{V_{h \mu \mathbf{p}-\mathbf{k}}^{2}}{\omega-E_{h \mu \mathbf{p}-\mathbf{k}}-\omega_{\nu \mathbf{p}}}\right)\right], \\
\Gamma_{L}^{\dagger}(\mathbf{k}, \omega) & =\frac{1}{8 N} \sum_{\mathbf{p}} \sum_{\mu \nu} Z_{h F A}^{(\mu)} \frac{\bar{\Delta}_{h z}^{(\mu)}(\mathbf{p}-\mathbf{k})}{2 E_{h \mu \mathbf{p}-\mathbf{k}}} \frac{B_{\nu \mathbf{p}}}{\omega_{\nu \mathbf{p}}} \\
& \times\left[L_{\mu \nu}^{(1)}(\mathbf{k}, \mathbf{p})\left(\frac{1.62 \mathrm{~b})}{\omega-E_{h \mu \mathbf{p}-\mathbf{k}}+\omega_{\nu \mathbf{p}}}-\frac{1}{\omega+E_{h \mu \mathbf{p}-\mathbf{k}}-\omega_{\nu \mathbf{p}}}\right)\right. \\
& \left.+L_{\mu \nu}^{(2)}(\mathbf{k}, \mathbf{p})\left(\frac{1}{\omega-E_{h \mu \mathbf{p}-\mathbf{k}}-\omega_{\nu \mathbf{p}}}-\frac{1}{\omega+E_{h \mu \mathbf{p}-\mathbf{k}}+\omega_{\nu \mathbf{p}}}\right)\right],(1.62 \mathrm{c}) \\
\Gamma_{T}^{\dagger}(\mathbf{k}, \omega) & =\frac{1}{8 N} \sum_{\mathbf{p}} \sum_{\mu \nu}(-1)^{\mu+\nu} Z_{h F A}^{(\mu)} \frac{\bar{\Delta}_{h z}^{(\mu)}(\mathbf{p}-\mathbf{k})}{2 E_{h \mu \mathbf{p}-\mathbf{k}}} \frac{B_{\nu \mathbf{p}}}{\omega_{\nu \mathbf{p}}} \\
& \times\left[L_{\mu \nu}^{(1)}(\mathbf{k}, \mathbf{p})\left(\frac{1}{\omega-E_{h \mu \mathbf{p}-\mathbf{k}}+\omega_{\nu \mathbf{p}}}-\frac{1}{\omega+E_{h \mu \mathbf{p}-\mathbf{k}}-\omega_{\nu \mathbf{p}}}\right)\right. \\
& \left.+L_{\mu \nu}^{(2)}(\mathbf{k}, \mathbf{p})\left(\frac{1}{\omega-E_{h \mu \mathbf{p}-\mathbf{k}}-\omega_{\nu \mathbf{p}}}-\frac{1}{\omega+E_{h \mu \mathbf{p}-\mathbf{k}}+\omega_{\nu \mathbf{p}}}\right)\right],(1.62 \mathrm{~d})
\end{aligned}
$$

where $L_{\mu \nu}^{(1)}(\mathbf{k}, \mathbf{p})=\left[\operatorname{coth}\left(\beta \omega_{\nu \mathbf{p}} / 2\right)-\tanh \left(\beta E_{h \mu \mathbf{p}-\mathbf{k}} / 2\right)\right] / 2$ and $L_{\mu \nu}^{(2)}(\mathbf{k}, \mathbf{p})=\left[\operatorname{coth}\left(\beta \omega_{\nu \mathbf{p}} / 2\right)\right.$ $\left.+\tanh \left(\beta E_{h \mu \mathbf{p}-\mathbf{k}} / 2\right)\right] / 2$, and the dressed holon effective gap parameters and quasiparticle coherent weights satisfy the following four equations,

$$
\begin{aligned}
\bar{\Delta}_{h L} & =-\frac{4}{32 N^{3}} \sum_{\mathbf{k}, \mathbf{q}, \mathbf{p}} \sum_{\nu, \nu^{\prime}, \nu^{\prime \prime}} \gamma_{\mathbf{k}-\mathbf{p}+\mathbf{q}}^{(d)} C_{\nu \nu^{\prime \prime}}(\mathbf{k}+\mathbf{q}) \frac{Z_{h F A}^{\left(\nu^{\prime \prime}\right)} B_{\nu^{\prime} \mathbf{p}} B_{\nu \mathbf{q}}}{\omega_{\nu^{\prime} \mathbf{p}} \omega_{\nu \mathbf{q}}} \bar{\Delta}_{h z}^{\left(\nu^{\prime \prime}\right)}(\mathbf{k}) \\
& \times\left(\frac{F_{\nu \nu^{\prime} \nu^{\prime \prime}}^{(1)}(\mathbf{q}, \mathbf{p})+F_{\nu \nu^{\prime} \nu^{\prime \prime}}(\mathbf{k}, \mathbf{q}, \mathbf{p})}{\left[\omega_{\nu^{\prime} \mathbf{p}}-\omega_{\nu \mathbf{q}}\right]^{2}-E_{h \nu^{\prime \prime} \mathbf{k}}^{2}}\right. \\
& \left.+\frac{F_{\nu \nu^{\prime} \nu^{\prime \prime}}^{(3)}(\mathbf{q}, \mathbf{p})+F_{\nu \nu^{\prime} \nu^{\prime \prime}}^{(4)}(\mathbf{k}, \mathbf{q}, \mathbf{p})}{\left[\omega_{\nu^{\prime} \mathbf{p}}+\omega_{\nu \mathbf{q}}\right]^{2}-E_{h \nu^{\prime \prime} \mathbf{k}}^{2}}\right) \\
\bar{\Delta}_{h T} & =-\frac{1}{32 N^{3}} \sum_{\mathbf{k}, \mathbf{q}, \mathbf{p} \nu, \nu^{\prime}, \nu^{\prime \prime}}(-1)^{\nu+\nu^{\prime}+\nu^{\prime \prime}+1} C_{\nu \nu^{\prime \prime}}(\mathbf{k}+\mathbf{q}) \frac{Z_{h F A}^{\left(\nu^{\prime \prime}\right)} B_{\nu^{\prime} \mathbf{p}} B_{\nu \mathbf{q}}}{\omega_{\nu^{\prime} \mathbf{p}} \omega_{\nu \mathbf{q}}} \bar{\Delta}_{h z}^{\left(\nu^{\prime \prime}\right)}(\mathbf{k}) \\
& \times\left(\frac{F_{\nu \nu^{\prime} \nu^{\prime \prime}}^{(1)}(\mathbf{q}, \mathbf{p})+F_{\nu \nu^{\prime} \nu^{\prime \prime}}^{(2)}(\mathbf{k}, \mathbf{q}, \mathbf{p})}{\left[\omega_{\nu^{\prime} \mathbf{p}}-\omega_{\nu \mathbf{q}}\right]^{2}-E_{h \nu^{\prime \prime} \mathbf{k}}^{2}}\right. \\
& \left.+\frac{F_{\nu \nu^{\prime} \nu^{\prime \prime}}^{(3)}(\mathbf{q}, \mathbf{p})+F_{\nu \nu^{\prime} \nu^{\prime \prime}}^{(4)}(\mathbf{k}, \mathbf{q}, \mathbf{p})}{\left[\omega_{\nu^{\prime} \mathbf{p}}+\omega_{\nu \mathbf{q}}\right]^{2}-E_{h \nu^{\prime \prime} \mathbf{k}}^{2}}\right)
\end{aligned}
$$




$$
\begin{aligned}
& \frac{1}{Z_{h F A}^{(1)}}=1+\frac{1}{32 N^{2}} \sum_{\mathbf{q}, \mathbf{p}} \sum_{\nu, \nu^{\prime}, \nu^{\prime \prime}}\left[1+(-1)^{\nu+\nu^{\prime}+\nu^{\prime \prime}+1}\right] C_{\nu \nu^{\prime \prime}}\left(\mathbf{p}+\mathbf{k}_{A}\right) \\
& \times \frac{Z_{h F A}^{\left(\nu^{\prime \prime}\right)} B_{\nu^{\prime} \mathbf{p}} B_{\nu \mathbf{q}}}{\omega_{\nu^{\prime} \mathbf{p}} \omega_{\nu \mathbf{q}}}\left(\frac{H_{\nu \nu^{\prime} \nu^{\prime \prime}}^{(1)}(\mathbf{q}, \mathbf{p})}{\left[\omega_{\nu^{\prime} \mathbf{p}}-\omega_{\nu \mathbf{q}}+E_{\left.h \nu^{\prime \prime} \mathbf{p}-\mathbf{q}+\mathbf{k}_{A}\right]^{2}}\right.}\right. \\
& +\frac{H_{\nu \nu^{\prime} \nu^{\prime \prime}}^{(2)}(\mathbf{q}, \mathbf{p})}{\left[\omega_{\nu^{\prime} \mathbf{p}}-\omega_{\nu \mathbf{q}}-E_{\left.h \nu^{\prime \prime} \mathbf{p}-\mathbf{q}+\mathbf{k}_{A}\right]^{2}}\right]^{2}}+\frac{H_{\nu \nu^{\prime} \nu^{\prime \prime}}^{(3)}(\mathbf{q}, \mathbf{p})}{\left[\omega_{\nu^{\prime} \mathbf{p}}+\omega_{\nu \mathbf{q}}+E_{\left.h \nu^{\prime \prime} \mathbf{p}-\mathbf{q}+\mathbf{k}_{A}\right]^{2}}\right.} \\
& +\frac{H_{\nu \nu^{\prime} \nu^{\prime \prime}}^{(4)}(\mathbf{q}, \mathbf{p})}{\left[\omega_{\nu^{\prime} \mathbf{p}}+\omega_{\nu \mathbf{q}}-E_{\left.h \nu^{\prime \prime} \mathbf{p}-\mathbf{q}+\mathbf{k}_{A}\right]^{2}}\right)}, \\
& \frac{1}{Z_{h F A}^{(2)}}=1+\frac{1}{32 N^{2}} \sum_{\mathbf{q}, \mathbf{p}} \sum_{\nu, \nu^{\prime}, \nu^{\prime \prime}}\left[1-(-1)^{\nu+\nu^{\prime}+\nu^{\prime \prime}+1}\right] C_{\nu \nu^{\prime \prime}}\left(\mathbf{p}+\mathbf{k}_{A}\right) \\
& \times \frac{Z_{h F A}^{\left(\nu^{\prime \prime}\right)} B_{\nu^{\prime} \mathbf{p}} B_{\nu \mathbf{q}}}{\omega_{\nu^{\prime} \mathbf{p}} \omega_{\nu \mathbf{q}}}\left(\frac{H_{\nu \nu^{\prime} \nu^{\prime \prime}}^{(1)}(\mathbf{q}, \mathbf{p})}{\left[\omega_{\nu^{\prime} \mathbf{p}}-\omega_{\nu \mathbf{q}}+E_{h \nu^{\prime \prime} \mathbf{p}-\mathbf{q}+\mathbf{k}_{A}}\right]^{2}}\right.
\end{aligned}
$$

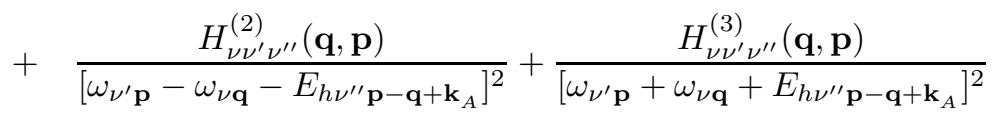

$$
\begin{aligned}
& \left.+\frac{H_{\nu \nu^{\prime} \nu^{\prime \prime}}^{(4)}(\mathbf{q}, \mathbf{p})}{\left[\omega_{\nu^{\prime} \mathbf{p}}+\omega_{\nu \mathbf{q}}-E_{\left.h \nu^{\prime \prime} \mathbf{p}-\mathbf{q}+\mathbf{k}_{A}\right]^{2}}\right.}\right),
\end{aligned}
$$

where $C_{\nu \nu^{\prime \prime}}(\mathbf{k})=\left[Z\left(t \gamma_{\mathbf{k}}-t^{\prime} \gamma_{\mathbf{k}}^{\prime}\right)+(-1)^{\nu+\nu^{\prime \prime}} t_{\perp}(\mathbf{k})\right]^{2}$, and

$$
\begin{aligned}
& F_{\nu \nu^{\prime} \nu^{\prime \prime}}^{(1)}(\mathbf{q}, \mathbf{p})=n_{B}\left(\omega_{\nu \mathbf{q}}\right)+n_{B}\left(\omega_{\nu^{\prime}} \mathbf{p}\right)+2 n_{B}\left(\omega_{\nu \mathbf{q}}\right) n_{B}\left(\omega_{\nu^{\prime}} \mathbf{p}\right) \\
& F_{\nu \nu^{\prime} \nu^{\prime \prime}}^{(2)}(\mathbf{k}, \mathbf{q}, \mathbf{p})=\frac{\omega_{\nu \mathbf{q}}-\omega_{\nu^{\prime}} \mathbf{p}}{E_{h \nu^{\prime \prime} \mathbf{k}}} \tanh \left[\frac{1}{2} \beta E_{h \nu^{\prime \prime} \mathbf{k}}\right]\left[n_{B}\left(\omega_{\nu \mathbf{q}}\right)-n_{B}\left(\omega_{\nu^{\prime} \mathbf{p}}\right)\right], \\
& F_{\nu \nu^{\prime} \nu^{\prime \prime}}^{(3)}(\mathbf{q}, \mathbf{p})=\left[1+n_{B}\left(\omega_{\nu \mathbf{q}}\right)\right]\left[1+n_{B}\left(\omega_{\nu^{\prime} \mathbf{p}}\right)\right]+n_{B}\left(\omega_{\nu \mathbf{q}}\right) n_{B}\left(\omega_{\nu^{\prime} \mathbf{p}}\right), \\
& F_{\nu \nu^{\prime} \nu^{\prime \prime}}^{(4)}(\mathbf{k}, \mathbf{q}, \mathbf{p})=\frac{\omega_{\nu^{\prime} \mathbf{p}}+\omega_{\nu \mathbf{q}}}{-E_{h \nu^{\prime \prime} \mathbf{k}}} \tanh \left[\frac{1}{2} \beta E_{h \nu^{\prime \prime} \mathbf{k}}\right]\left[1+n_{B}\left(\omega_{\nu \mathbf{q}}\right)+n_{B}\left(\omega_{\nu^{\prime} \mathbf{p}}\right)\right\}_{1}
\end{aligned}
$$

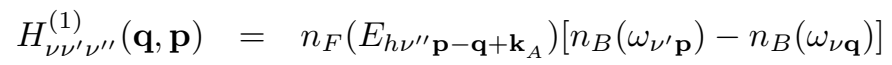

$$
\begin{aligned}
& +n_{B}\left(\omega_{\nu \mathbf{q}}\right)\left[1+n_{B}\left(\omega_{\nu^{\prime} \mathbf{p}}\right)\right] \text {, } \\
& H_{\nu \nu^{\prime} \nu^{\prime \prime}}^{(2)}(\mathbf{q}, \mathbf{p})=n_{F}\left(E_{\left.h \nu^{\prime \prime} \mathbf{p}-\mathbf{q}+\mathbf{k}_{A}\right)}\right)\left[n_{B}\left(\omega_{\nu \mathbf{q}}\right)-n_{B}\left(\omega_{\nu^{\prime} \mathbf{p}}\right)\right] \\
& +n_{B}\left(\omega_{\nu^{\prime} \mathbf{p}}\right)\left[1+n_{B}\left(\omega_{\nu \mathbf{q}}\right)\right] \text {, } \\
& H_{\nu \nu^{\prime} \nu^{\prime \prime}}^{(3)}(\mathbf{q}, \mathbf{p})=\left[1-n_{F}\left(E_{h \nu^{\prime \prime} \mathbf{p}-\mathbf{q}+\mathbf{k}_{A}}\right)\right]\left[1+n_{B}\left(\omega_{\nu \mathbf{q}}\right)+n_{B}\left(\omega_{\nu^{\prime}} \mathbf{p}\right)\right] \\
& +n_{B}\left(\omega_{\nu \mathbf{q}}\right) n_{B}\left(\omega_{\nu^{\prime} \mathbf{p}}\right) \text {, }
\end{aligned}
$$

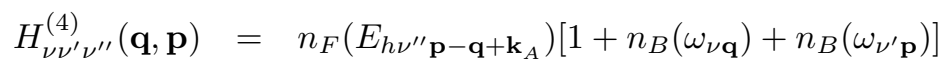

$$
\begin{aligned}
& +n_{B}\left(\omega_{\nu \mathbf{q}}\right) n_{B}\left(\omega_{\nu^{\prime} \mathbf{p}}\right) \text {. }
\end{aligned}
$$

As in the single layer case [35, 32 discussed in section 3, these four equations 
must be solved self-consistently in combination with other equations,

$$
\begin{aligned}
& \delta=\frac{1}{4 N} \sum_{\nu, \mathbf{k}} Z_{h F A}^{(\nu)}\left(1-\frac{\bar{\xi}_{\nu \mathbf{k}}}{E_{h \nu \mathbf{k}}} \tanh \left[\frac{1}{2} \beta E_{h \nu \mathbf{k}}\right]\right), \\
& \phi_{1}=\frac{1}{4 N} \sum_{\nu, \mathbf{k}} \gamma_{\mathbf{k}} Z_{h F A}^{(\nu)}\left(1-\frac{\bar{\xi}_{\nu \mathbf{k}}}{E_{h \nu \mathbf{k}}} \tanh \left[\frac{1}{2} \beta E_{h \nu \mathbf{k}}\right]\right), \\
& \phi_{2}=\frac{1}{4 N} \sum_{\nu, \mathbf{k}} \gamma_{\mathbf{k}}^{\prime} Z_{h F A}^{(\nu)}\left(1-\frac{\bar{\xi}_{\nu \mathbf{k}}}{E_{h \nu \mathbf{k}}} \tanh \left[\frac{1}{2} \beta E_{h \nu \mathbf{k}}\right]\right), \\
& \phi_{\perp}=\frac{1}{4 N} \sum_{\nu, \mathbf{k}}(-1)^{\nu+1} Z_{h F A}^{(\nu)}\left(1-\frac{\bar{\xi}_{\nu \mathbf{k}}}{E_{h \nu \mathbf{k}}} \tanh \left[\frac{1}{2} \beta E_{h \nu \mathbf{k}}\right]\right), \\
& \frac{1}{2}=\frac{1}{4 N} \sum_{\nu, \mathbf{k}} \frac{B_{\nu \mathbf{k}}}{\omega_{\nu \mathbf{k}}} \operatorname{coth}\left[\frac{1}{2} \beta \omega_{\nu \mathbf{k}}\right] \\
& \chi_{1}=\frac{1}{4 N} \sum_{\nu, \mathbf{k}} \gamma_{\mathbf{k}} \frac{B_{\nu \mathbf{k}}}{\omega_{\nu \mathbf{k}}} \operatorname{coth}\left[\frac{1}{2} \beta \omega_{\nu \mathbf{k}}\right] \\
& \chi_{2}=\frac{1}{4 N} \sum_{\nu, \mathbf{k}} \gamma^{\prime} \mathbf{k} \frac{B_{\nu \mathbf{k}}}{\omega_{\nu \mathbf{k}}} \operatorname{coth}\left[\frac{1}{2} \beta \omega_{\nu \mathbf{k}}\right] \text {, } \\
& C_{1}=\frac{1}{4 N} \sum_{\nu, \mathbf{k}} \gamma_{\mathbf{k}}^{2} \frac{B_{\nu \mathbf{k}}}{\omega_{\nu \mathbf{k}}} \operatorname{coth}\left[\frac{1}{2} \beta \omega_{\nu \mathbf{k}}\right] \\
& C_{2}=\frac{1}{4 N} \sum_{\nu, \mathbf{k}} \gamma_{\mathbf{k}} \gamma^{\prime} \mathbf{k} \frac{B_{\nu \mathbf{k}}}{\omega_{\nu \mathbf{k}}} \operatorname{coth}\left[\frac{1}{2} \beta \omega_{\nu \mathbf{k}}\right], \\
& C_{3}=\frac{1}{4 N} \sum_{\nu, \mathbf{k}} \gamma^{\prime}{ }_{\mathbf{k}} \frac{B_{\nu \mathbf{k}}}{\omega_{\nu \mathbf{k}}} \operatorname{coth}\left[\frac{1}{2} \beta \omega_{\nu \mathbf{k}}\right] \text {, } \\
& \chi_{1}^{z}=\frac{1}{4 N} \sum_{\nu, \mathbf{k}} \gamma_{\mathbf{k}} \frac{B_{z \nu \mathbf{k}}}{\omega_{z \nu \mathbf{k}}} \operatorname{coth}\left[\frac{1}{2} \beta \omega_{z \nu \mathbf{k}}\right] \text {, } \\
& \chi_{2}^{z}=\frac{1}{4 N} \sum_{\nu, \mathbf{k}} \gamma^{\prime} \mathbf{k} \frac{B_{z \nu \mathbf{k}}}{\omega_{z \nu \mathbf{k}}} \operatorname{coth}\left[\frac{1}{2} \beta \omega_{z \nu \mathbf{k}}\right], \\
& C_{1}^{z}=\frac{1}{4 N} \sum_{\nu, \mathbf{k}} \gamma_{\mathbf{k}}^{2} \frac{B_{z \nu \mathbf{k}}}{\omega_{z \nu \mathbf{k}}} \operatorname{coth}\left[\frac{1}{2} \beta \omega_{z \nu \mathbf{k}}\right], \\
& C_{2}^{z}=\frac{1}{4 N} \sum_{\nu, \mathbf{k}} \gamma_{\mathbf{k}} \gamma^{\prime} \mathbf{k} \frac{B_{z \nu \mathbf{k}}}{\omega_{z \nu \mathbf{k}}} \operatorname{coth}\left[\frac{1}{2} \beta \omega_{z \nu \mathbf{k}}\right], \\
& \chi_{\perp}=\frac{1}{4 N} \sum_{\nu, \mathbf{k}}(-1)^{\nu+1} \frac{B_{\nu \mathbf{k}}}{\omega_{\nu \mathbf{k}}} \operatorname{coth}\left[\frac{1}{2} \beta \omega_{\nu \mathbf{k}}\right], \\
& C_{\perp}=\frac{1}{4 N} \sum_{\nu, \mathbf{k}}(-1)^{\nu+1} \gamma_{\mathbf{k}} \frac{B_{\nu \mathbf{k}}}{\omega_{\nu \mathbf{k}}} \operatorname{coth}\left[\frac{1}{2} \beta \omega_{\nu \mathbf{k}}\right], \\
& C^{\prime}{ }_{\perp}=\frac{1}{4 N} \sum_{\nu, \mathbf{k}}(-1)^{\nu+1} \gamma_{\mathbf{k}}^{\prime} \frac{B_{\nu \mathbf{k}}}{\omega_{\nu \mathbf{k}}} \operatorname{coth}\left[\frac{1}{2} \beta \omega_{\nu \mathbf{k}}\right]
\end{aligned}
$$




$$
\begin{aligned}
\chi_{\perp}^{z} & =\frac{1}{4 N} \sum_{\nu, \mathbf{k}}(-1)^{\nu+1} \frac{B_{z \nu \mathbf{k}}}{\omega_{z \nu \mathbf{k}}} \operatorname{coth}\left[\frac{1}{2} \beta \omega_{z \nu \mathbf{k}}\right], \\
C_{\perp}^{z} & =\frac{1}{4 N} \sum_{\nu, \mathbf{k}}(-1)^{\nu+1} \gamma_{\mathbf{k}} \frac{B_{z \nu \mathbf{k}}}{\omega_{z \nu \mathbf{k}}} \operatorname{coth}\left[\frac{1}{2} \beta \omega_{z \nu \mathbf{k}}\right], \\
C_{\perp}^{\prime z} & =\frac{1}{4 N} \sum_{\nu, \mathbf{k}}(-1)^{\nu+1} \gamma^{\prime}{ }_{\mathbf{k}} \frac{B_{z \nu \mathbf{k}}}{\omega_{z \nu \mathbf{k}}} \operatorname{coth}\left[\frac{1}{2} \beta \omega_{z \nu \mathbf{k}}\right],
\end{aligned}
$$

then all order parameters, decoupling parameter $\alpha$, and chemical potential $\mu$ are determined by the self-consistent calculation [37.

From the electron normal and anomalous Green's functions in Eqs. (62), we obtain the longitudinal and transverse parts of the electron spectral function and superconducting gap function as,

$$
\begin{aligned}
& A_{L}(\mathbf{k}, \omega)=\pi \frac{1}{4 N} \sum_{\mathbf{p}} \sum_{\mu \nu} Z_{h F A}^{(\mu)} \frac{B_{\nu \mathbf{p}}}{\omega_{\nu \mathbf{p}}}\left\{L _ { \mu \nu } ^ { ( 1 ) } ( \mathbf { k } , \mathbf { p } ) \left[U_{h \mu \mathbf{p}-\mathbf{k}}^{2} \delta\left(\omega+E_{h \mu \mathbf{p}-\mathbf{k}}-\omega_{\nu \mathbf{p}}\right)\right.\right. \\
& \left.+V_{h \mu \mathbf{p}-\mathbf{k}}^{2} \delta\left(\omega-E_{h \mu \mathbf{p}-\mathbf{k}}+\omega_{\nu \mathbf{p}}\right)\right] \\
& +L_{\mu \nu}^{(2)}(\mathbf{k}, \mathbf{p})\left[U_{h \mu \mathbf{p}-\mathbf{k}}^{2} \delta\left(\omega+E_{h \mu \mathbf{p}-\mathbf{k}}+\omega_{\nu \mathbf{p}}\right)\right. \\
& \left.\left.+V_{h \mu \mathbf{p}-\mathbf{k}}^{2} \delta\left(\omega-E_{h \mu \mathbf{p}-\mathbf{k}}-\omega_{\nu \mathbf{p}}\right)\right]\right\}, \\
& A_{T}(\mathbf{k}, \omega)=\pi \frac{1}{4 N} \sum_{\mathbf{p}} \sum_{\mu \nu}(-1)^{\mu+\nu} Z_{h F A}^{(\mu)} \frac{B_{\nu \mathbf{p}}}{\omega_{\nu \mathbf{p}}} \\
& \times\left\{L _ { \mu \nu } ^ { ( 1 ) } ( \mathbf { k } , \mathbf { p } ) \left[U_{h \mu \mathbf{p}-\mathbf{k}}^{2} \delta\left(\omega+E_{h \mu \mathbf{p}-\mathbf{k}}-\omega_{\nu \mathbf{p}}\right)\right.\right. \\
& \left.+V_{h \mu \mathbf{p}-\mathbf{k}}^{2} \delta\left(\omega-E_{h \mu \mathbf{p}-\mathbf{k}}+\omega_{\nu \mathbf{p}}\right)\right] \\
& +L_{\mu \nu}^{(2)}(\mathbf{k}, \mathbf{p})\left[U_{h \mu \mathbf{p}-\mathbf{k}}^{2} \delta\left(\omega+E_{h \mu \mathbf{p}-\mathbf{k}}+\omega_{\nu \mathbf{p}}\right)\right. \\
& \left.\left.+V_{h \mu \mathbf{p}-\mathbf{k}}^{2} \delta\left(\omega-E_{h \mu \mathbf{p}-\mathbf{k}}-\omega_{\nu \mathbf{p}}\right)\right]\right\} \text {, } \\
& \Delta_{L}(\mathbf{k})=-\frac{1}{16 N} \sum_{\mathbf{p}, \mu, \nu} Z_{h F A}^{(\mu)} \frac{\bar{\Delta}_{h z}^{(\mu)}(\mathbf{p}-\mathbf{k})}{E_{h \mu \mathbf{p}-\mathbf{k}}} \\
& \times \frac{B_{\nu \mathbf{p}}}{\omega_{\nu \mathbf{p}}} \tanh \left[\frac{1}{2} \beta E_{h \mu \mathbf{p}-\mathbf{k}}\right] \operatorname{coth}\left[\frac{1}{2} \beta \omega_{\nu \mathbf{p}}\right], \\
& \Delta_{T}(\mathbf{k})=-\frac{1}{16 N} \sum_{\mathbf{p}, \mu, \nu}(-1)^{\mu+\nu} Z_{h F A}^{(\mu)} \frac{\bar{\Delta}_{h z}^{(\mu)}(\mathbf{p}-\mathbf{k})}{E_{h \mu \mathbf{p}-\mathbf{k}}} \\
& \times \frac{B_{\nu \mathbf{p}}}{\omega_{\nu \mathbf{p}}} \tanh \left[\frac{1}{2} \beta E_{h \mu \mathbf{p}-\mathbf{k}}\right] \operatorname{coth}\left[\frac{1}{2} \beta \omega_{\nu \mathbf{p}}\right] .
\end{aligned}
$$

With the help of these longitudinal and transverse parts of the superconducting gap functions in Eqs. (66c) and (66d), the corresponding longitudinal and transverse superconducting gap parameters are obtained as $\Delta_{L}=-\chi_{1} \Delta_{h L}$ and $\Delta_{T}=-\chi_{\perp} \Delta_{h T}$, respectively. In the bilayer coupling case, the more appropriate classification is in terms of the spectral function and superconducting gap function within the basis of the antibonding and bonding components 


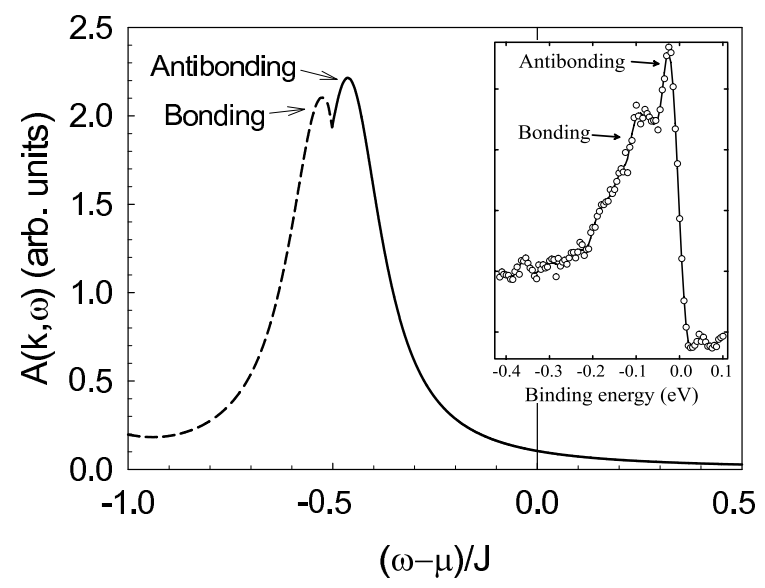

Figure 1.8: The antibonding (solid line) and bonding (dashed line) electron spectral functions of the bilayer cuprate superconductors at the $[\pi, 0]$ point in the superconducting state for $t / J=2.5, t^{\prime} / t=0.3$, and $t_{\perp} / t=0.35$ with $T=$ $0.002 J$ at $\delta=0.15$. Inset: the corresponding ARPES experimental result 77] of the bilayer cuprate superconductor $\mathrm{Bi}_{2} \mathrm{Sr}_{2} \mathrm{CaCu}_{2} \mathrm{O}_{8+\delta}$ in the superconducting state.

25, 74, 75, 76, 77]. In this case, the electron spectral function and superconducting gap parameter can be transformed from the plane representation to the antibonding-bonding representation as,

$$
\begin{aligned}
A^{(a)}(\mathbf{k}, \omega) & =\frac{1}{2}\left[A_{L}(\mathbf{k}, \omega)-A_{T}(\mathbf{k}, \omega)\right], \\
A^{(b)}(\mathbf{k}, \omega) & =\frac{1}{2}\left[A_{L}(\mathbf{k}, \omega)+A_{T}(\mathbf{k}, \omega)\right], \\
\Delta^{(a)} & =\Delta_{L}-\Delta_{T} \\
\Delta^{(b)} & =\Delta_{L}+\Delta_{T} .
\end{aligned}
$$

respectively, then the antibonding and bonding parts have odd and even symmetries, respectively.

In Fig. 8, we firstly plot the antibonding (solid line) and bonding (dashed line) electron spectral functions in the $[\pi, 0]$ point for $t / J=2.5, t^{\prime} / t=0.3$, and $t_{\perp} / t=0.35$ with $T=0.002 J$ at $\delta=0.15$. For comparison, the corresponding ARPES experimental result [77] of the bilayer cuprate superconductor $\mathrm{Bi}_{2} \mathrm{Sr}_{2} \mathrm{CaCu}_{2} \mathrm{O}_{8+\delta}$ in the superconducting state is also shown in Fig. 8 (inset). In comparison with the single layer case [35] in section 3, the electron spectrum of the bilayer system has been split into the bonding and antibonging components, with the bonding and antibonding superconducting quasiparticle peaks in the $[\pi, 0]$ point are located at the different positions. In this sense, the differentiation between the bonding and antibonding components of the electron spectral function is essential. The antibonding spectrum consists of a low energy 


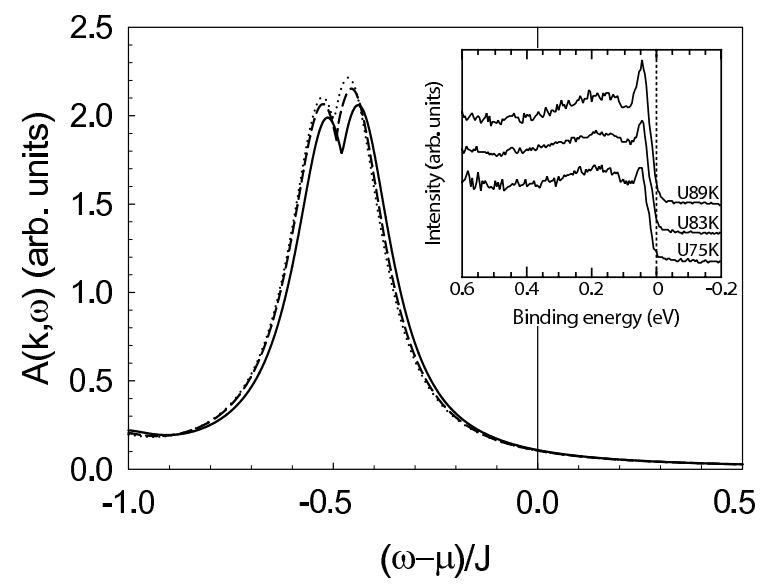

Figure 1.9: The electron spectral functions of the bilayer cuprate superconductors at $[\pi, 0]$ point in the superconducting state for $t / J=2.5, t^{\prime} / t=0.3$, and $t_{\perp} / t=0.35$ with $T=0.002 J$ at $\delta=0.09$ (solid line), $\delta=0.12$ (dashed line), and $\delta=0.15$ (dotted line). Inset: the corresponding ARPES experimental results [64] of the bilayer cuprate superconductor $\mathrm{Bi}_{2} \mathrm{Sr}_{2} \mathrm{CaCu}_{2} \mathrm{O}_{8+\delta}$ in the superconducting state.

antibonding peak, corresponding to the superconducting peak, and the bonding spectrum has a higher energy bonding peak, corresponding to the hump, while the spectral dip is in between them, then the total contributions for the electron spectrum from both antibonding and bonding components give rise to the peak-dip-hump structure, in qualitative agreement with the experimental observation on the bilayer cuprate superconductors in the superconducting state [4, 11, 21, 22, 23, 24.

We now turn to discuss the doping evolution of the electron spectrum of bilayer cuprate superconductors in the superconducting state. We have calculated the electron spectrum at different doping concentrations, and the result of the electron spectral functions at the $[\pi, 0]$ point for $t / J=2.5, t^{\prime} / t=0.3$, and $t_{\perp} / t=0.35$ with $T=0.002 J$ at $\delta=0.09$ (solid line), $\delta=0.12$ (dashed line), and $\delta=0.15$ (dotted line) are plotted in Fig. 9 in comparison with the corresponding ARPES experimental results 64] of the bilayer cuprate superconductor $\mathrm{Bi}_{2} \mathrm{Sr}_{2} \mathrm{CaCu}_{2} \mathrm{O}_{8+\delta}$ in the superconducting state (inset). Obviously, the doping evolution of the spectral weight of the bilayer superconductor $\mathrm{Bi}_{2} \mathrm{Sr}_{2} \mathrm{CaCu}_{2} \mathrm{O}_{8+\delta}$ in the superconducting state is reproduced. With increasing the doping concentration, both superconducting peak and hump become sharper, and then the spectral weights increase in intensity. Furthermore, we have also calculated the electron spectrum with different temperatures, and the results show that the spectral weights of both superconducting peak and hump are suppressed with increasing temperatures. These results are also qualitatively consistent with the ARPES experimental results on the bilayer cuprate superconductors in the 


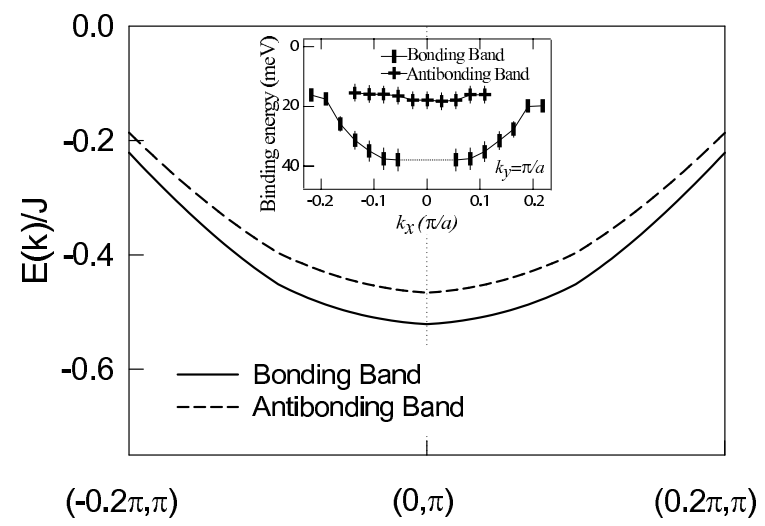

Figure 1.10: The positions of the antibonding peaks and bonding humps in the electron spectrum of the bilayer cuprate superconductors in the superconducting state as a function of momentum along the direction $[-0.2 \pi, \pi] \rightarrow[0, \pi] \rightarrow$ $[0.2 \pi, \pi]$ with $T=0.002 J$ at $\delta=0.15$ for $t / J=2.5, t^{\prime} / t=0.3$, and $t_{\perp} / t=$ 0.35. Inset: the corresponding experimental data [74 of the bilayer cuprate superconductor $\mathrm{Bi}_{2} \mathrm{Sr}_{2} \mathrm{CaCu}_{2} \mathrm{O}_{8+\delta}$ in the superconducting state.

superconducting state [4, 22, 64].

To better perceive the superconducting coherence of the antibonding quasiparticle peak and bonding quasiparticle hump around the $[\pi, 0]$ point, we have made a series of calculations for the electron spectral function at different momenta, and the result shows that the sharp superconducting peak from the electron antibonding spectral function and hump from the bonding spectral function persist in a very large momentum space region around the $[\pi, 0]$ point. To show this point clearly, we plot the positions of the antibonding peak and bonding hump in the electron spectrum as a function of momentum along the direction $[-0.2 \pi, \pi] \rightarrow[0, \pi] \rightarrow[0.2 \pi, \pi]$ with $T=0.002 J$ at $\delta=0.15$ for $t / J=2.5$, $t^{\prime} / t=0.3$, and $t_{\perp} / t=0.35$ in Fig. 10 in comparison with the corresponding experimental data [74] of the bilayer cuprate superconductor $\mathrm{Bi}_{2} \mathrm{Sr}_{2} \mathrm{CaCu}_{2} \mathrm{O}_{8+\delta}$ in the superconducting state (inset). It is shown that there are two branches in the quasiparticle dispersion, with upper branch corresponding to the antibonding quasiparticle dispersion, and lower branch corresponding to the bonding quasiparticle dispersion. Furthermore, the bilayer splitting reaches its maximum at the $[\pi, 0]$ point, in qualitative agreement with the ARPES experimental measurements on the bilayer cuprate superconductors in the superconducting state [4, 11, 21, 22, 23, 24.

In the above calculations for the bilayer systems, we find that although the antibonding superconducting peak and bonding hump have different dispersions, the transverse part of the superconducting gap parameter $\Delta_{T} \approx 0$. To show this point clearly, we plot the antibonding and bonding gap parameters in Eqs. (67c) and (67d) as a function of the doping concentration with $T=0.002 J$ 


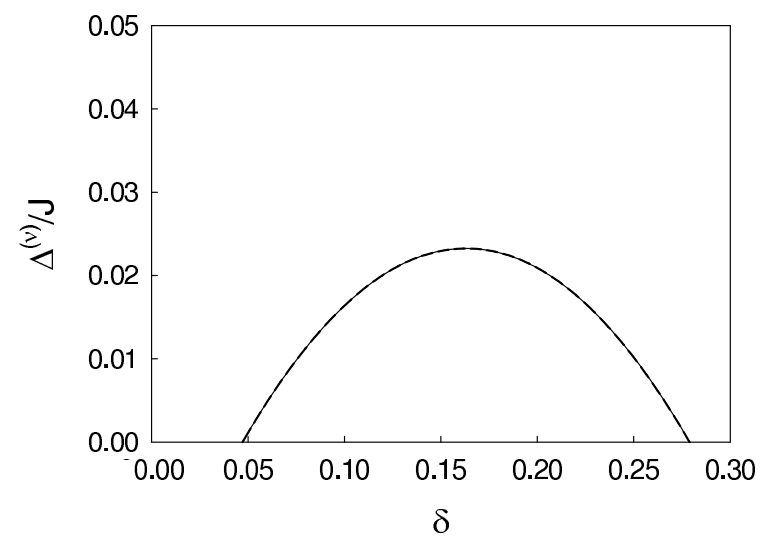

Figure 1.11: The antibonding (solid line) and bonding (dashed line) superconducting gap parameters of the bilayer cuprate superconductors as a function of the doping concentration with $T=0.002 J$ for $t / J=2.5, t^{\prime} / t=0.3$, and $t_{\perp} / t=0.35$.

for $t / J=2.5, t^{\prime} / t=0.3$, and $t_{\perp} / t=0.35$ in Fig. 11. As seen from Fig. 11, both antibonding and bonding gap parameters have the same d-wave superconducting gap magnitude in a given doping concentration, i.e., $\Delta^{a} \approx \Delta^{b}$. This result shows that although there is a single electron interlayer coherent hopping (49) in the bilayer cuprate superconductors in the superconducting state, the electron interlayer pairing interaction vanishes. This reflects that in the kinetic energy driven superconducting mechanism, the weak dressed holon-spin interaction due to the interlayer coherent hopping (49) from the kinetic energy term of the bilayer $t-J$ model does not induce the dressed holon interlayer pairing state by exchanging spin excitations in the higher power of the doping concentration. This is different from the dressed holon-spin interaction due to the intralayer hopping from the kinetic energy term of the bilayer $t-J$ model, it can induce superconductivity by exchanging spin excitations in the higher power of the doping concentration [30. This result is also consistent with the ARPES experimental results of the bilayer cuprate superconductor $\mathrm{Bi}(\mathrm{Pb})_{2} \mathrm{Sr}_{2} \mathrm{CaCu}_{2} \mathrm{O}_{8+\delta}$ in superconducting state [74, 77, where the superconducting gap separately for the bonding and antibonding bands has been measured, and it is found that both d-wave superconducting gaps from the antibonding and bonding components are identical within the experimental uncertainties.

Now we give some physical interpretation to the above obtained results. We find that there are two main reasons why the electronic structure of the bilayer cuprate superconductors in the superconducting state can be described qualitatively in the framework of the kinetic energy driven superconductivity by considering the bilayer interaction: Firstly, the bilayer interaction causes the bilayer slitting, this leads to that the full electron normal (anomalous) Green's function is divided into the longitudinal and transverse parts, respectively, then 
the bonding and antibonding electron spectral functions (superconducting gap functions) are obtained from these longitudinal and transverse parts of the electron normal (anomalous) Green's function, respectively. Although the transverse part of the superconducting gap parameter $\Delta_{T} \approx 0$, the antibonding peak around the $[\pi, 0]$ point is always at lower binding energy than the bonding peak (hump) due to the bilayer slitting. In this sense, the peak-dip-hump structure in the bilayer cuprate superconductors in the superconducting state is mainly caused by the bilayer splitting. Secondly, the superconducting state in the bilayer cuprate superconductors is the conventional BCS like with the d-wave symmetry as in the single layer case 35,32 discussed in section 3. This can be understood from the electron normal and anomalous Green's functions in Eqs. (62). Since the spins center around the $[\pi, \pi]$ point in the mean-field level [35, 30, 37, then the main contributions for the spins comes from the $[\pi, \pi]$ point. In this case, the longitudinal and transverse parts of the electron normal and anomalous Green's functions in Eqs. (62) can be approximately reduced in terms of $\omega_{\nu \mathbf{p}=[\pi, \pi]} \sim 0$ and one of the self-consistent equations in Eq. (65e) as 37,

$$
\begin{aligned}
G_{L}(\mathbf{k}, \omega) & \approx \frac{1}{2} \sum_{\nu=1,2} Z_{F A}^{(\nu)}\left(\frac{U_{\nu \mathbf{k}}^{2}}{\omega-E_{\nu \mathbf{k}}}+\frac{V_{\nu \mathbf{k}}^{2}}{\omega+E_{\nu \mathbf{k}}}\right) \\
G_{T}(\mathbf{k}, \omega) & \approx \frac{1}{2} \sum_{\nu=1,2}(-1)^{\nu+1} Z_{F A}^{(\nu)}\left(\frac{U_{\nu \mathbf{k}}^{2}}{\omega-E_{\nu \mathbf{k}}}+\frac{V_{\nu \mathbf{k}}^{2}}{\omega+E_{\nu \mathbf{k}}}\right) \\
\Gamma_{L}^{\dagger}(\mathbf{k}, \omega) & =\frac{1}{2} \sum_{\nu=1,2} Z_{F A}^{(\nu)} \frac{\bar{\Delta}_{h z}^{(\nu)}(\mathbf{k})}{2 E_{\nu \mathbf{k}}}\left(\frac{1}{\omega-E_{\nu \mathbf{k}}}-\frac{1}{\omega+E_{\nu \mathbf{k}}}\right) \\
\Gamma_{T}^{\dagger}(\mathbf{k}, \omega) & =\frac{1}{2} \sum_{\nu=1,2}(-1)^{\nu+1} Z_{F A}^{(\nu)} \frac{\bar{\Delta}_{h z}^{(\nu)}(\mathbf{k})}{2 E_{\nu \mathbf{k}}}\left(\frac{1}{\omega-E_{\nu \mathbf{k}}}-\frac{1}{\omega+E_{\nu \mathbf{k}}}\right)
\end{aligned}
$$

with the electron quasiparticle coherence factors,

$$
\begin{aligned}
U_{\nu \mathbf{k}}^{2} & \approx V_{h \nu \mathbf{k}-\mathbf{k}_{A F}}^{2}, \\
V_{\nu \mathbf{k}}^{2} & \approx U_{h \nu \mathbf{k}-\mathbf{k}_{A F}}^{2},
\end{aligned}
$$

where the electron coherent weights $Z_{F A}^{(\nu)}=Z_{h F A}^{(\nu)} / 2$, and the electron quasiparticle spectrum $E_{\nu \mathbf{k}} \approx E_{h \nu \mathbf{k}-\mathbf{k}_{\mathbf{A F}}}$. As a result, these electron normal and anomalous Green's functions in Eq. (68) are typical bilayer BCS formalism with the d-wave gap function [19. In this sense, the peak-dip-hump structure in the bilayer cuprate superconductors is unrelated to superconductivity.

\subsubsection{Electronic structure of the bilayer cuprate supercon- ductors in the normal state}

In this subsection, we discuss the electronic structure of the bilayer cuprate superconductors in the normal state [36]. As the discussions of the electronic 
structure of the single layer case in section 3 , the dressed holon longitudinal and transverse parts of the pairing order parameter of the bilayer cuprate superconductors $\Delta_{h L}=0$ and $\Delta_{h T}=0$ (then the longitudinal and transverse parts of the superconducting gap parameter $\Delta_{L}=0$ and $\left.\Delta_{T}=0\right)$ in the normal state. In this case, the longitudinal and transverse parts of the full dressed holon normal Green's function in Eqs. (53a) and (53b) are reduced as [36],

$$
\begin{aligned}
g_{L}(\mathbf{k}, \omega) & =\frac{1}{2} \sum_{\nu=1,2} \frac{Z_{h F A}^{(\nu)}}{\omega-\bar{\xi}_{\nu \mathbf{k}}} \\
g_{T}(\mathbf{k}, \omega) & =\frac{1}{2} \sum_{\nu=1,2}(-1)^{\nu+1} \frac{Z_{h F A}^{(\nu)}}{\omega-\bar{\xi}_{\nu \mathbf{k}}}
\end{aligned}
$$

while the equations satisfied by the dressed holon quasiparticle coherent weights $Z_{h F A}^{(1)}$ and $Z_{h F A}^{(2)}$ in Eqs. (63c) and (63d) are reduced as,

$$
\begin{aligned}
\frac{1}{Z_{h F A}^{(1)}} & =1+\frac{1}{32 N^{2}} \sum_{\mathbf{q}, \mathbf{p}} \sum_{\nu, \nu^{\prime}, \nu^{\prime \prime}}\left[1+(-1)^{\nu+\nu^{\prime}+\nu^{\prime \prime}+1}\right] C_{\nu \nu^{\prime \prime}}\left(\mathbf{p}+\mathbf{k}_{N}\right) Z_{h F A}^{\left(\nu^{\prime \prime}\right)} \frac{B_{\nu^{\prime} \mathbf{p}} B_{\nu \mathbf{q}}}{\omega_{\nu^{\prime}} \mathbf{p} \omega_{\nu \mathbf{q}}} \\
& \times\left(\frac{R_{\nu \nu^{\prime} \nu^{\prime \prime}}(\mathbf{q}, \mathbf{p})}{\left[\omega_{\nu^{\prime} \mathbf{p}}-\omega_{\nu \mathbf{q}}+\bar{\xi}_{\left.\nu^{\prime \prime} \mathbf{p}-\mathbf{q}+\mathbf{k}_{N}\right]^{2}}\right.}+\frac{R_{\nu \nu^{\prime \prime}}(\mathbf{q}, \mathbf{p})}{\left[\omega_{\nu^{\prime} \mathbf{p}}-\omega_{\nu \mathbf{q}}-\bar{\xi}_{\left.\nu^{\prime \prime} \mathbf{p}-\mathbf{q}+\mathbf{k}_{N}\right]^{2}}\right.}\right. \\
& \left.+\frac{R_{\nu \nu^{\prime} \nu^{\prime \prime}}^{(3)}(\mathbf{q}, \mathbf{p})}{\left[\omega_{\nu^{\prime} \mathbf{p}}+\omega_{\nu \mathbf{q}}+\bar{\xi}_{\left.\nu^{\prime \prime} \mathbf{p}-\mathbf{q}+\mathbf{k}_{N}\right]^{2}}\right.}+\frac{R_{\nu \nu^{\prime} \nu^{\prime \prime}}^{(4)}(\mathbf{q}, \mathbf{p})}{\left[\omega_{\nu^{\prime} \mathbf{p}}+\omega_{\nu \mathbf{q}}-\bar{\xi}_{\nu^{\prime \prime} \mathbf{p}-\mathbf{q}+\mathbf{k}_{N}}\right]^{2}}\right),(1.71 \mathrm{a}) \\
\frac{1}{Z_{h F A}^{(2)}} & =1+\frac{1}{32 N^{2}} \sum_{\mathbf{q}, \mathbf{p}} \sum_{\nu, \nu^{\prime}, \nu^{\prime \prime}}\left[1-(-1)^{\nu+\nu^{\prime}+\nu^{\prime \prime}+1}\right] C_{\nu \nu^{\prime \prime}}\left(\mathbf{p}+\mathbf{k}_{N}\right) Z_{h F A}^{\left(\nu^{\prime \prime}\right)} \frac{B_{\nu^{\prime} \mathbf{p}} B_{\nu \mathbf{q}}}{\omega_{\nu^{\prime} \mathbf{p}} \omega_{\nu \mathbf{q}}} \\
& \times\left(\frac{R_{\nu \nu^{\prime} \nu^{\prime \prime}}(\mathbf{q}, \mathbf{p})}{\left[\omega_{\nu^{\prime} \mathbf{p}}-\omega_{\nu \mathbf{q}}+\bar{\xi}_{\left.\nu^{\prime \prime} \mathbf{p}-\mathbf{q}+\mathbf{k}_{N}\right]^{2}}\right.}+\frac{R_{\nu \nu^{\prime} \nu^{\prime \prime}}(\mathbf{q}, \mathbf{p})}{\left[\omega_{\nu^{\prime} \mathbf{p}}-\omega_{\nu \mathbf{q}}-\bar{\xi}_{\nu^{\prime \prime}} \mathbf{p}-\mathbf{q}+\mathbf{k}_{N}\right]^{2}}\right. \\
& \left.+\frac{R_{\nu \nu^{\prime} \nu^{\prime \prime}}^{(3)}(\mathbf{q}, \mathbf{p})}{\left[\omega_{\nu^{\prime} \mathbf{p}}+\omega_{\nu \mathbf{q}}+\bar{\xi}_{\nu^{\prime \prime} \mathbf{p}-\mathbf{q}+\mathbf{k}_{N}}\right]^{2}}+\frac{R_{\nu \nu^{\prime} \nu^{\prime \prime}}^{(4)}(\mathbf{q}, \mathbf{p})}{\left[\omega_{\nu^{\prime} \mathbf{p}}+\omega_{\nu \mathbf{q}}-\bar{\xi}_{\nu^{\prime \prime}} \mathbf{p}-\mathbf{q}+\mathbf{k}_{N}\right]^{2}}\right),(1.71 \mathrm{~b})
\end{aligned}
$$

with

$$
\begin{aligned}
& R_{\nu \nu^{\prime} \nu^{\prime \prime}}^{(1)}(\mathbf{q}, \mathbf{p})=n_{F}\left(\bar{\xi}_{\nu^{\prime \prime} \mathbf{p}-\mathbf{q}+\mathbf{k}_{N}}\right)\left[n_{B}\left(\omega_{\nu^{\prime} \mathbf{p}}\right)-n_{B}\left(\omega_{\nu \mathbf{q}}\right)\right] \\
& \text { - } n_{B}\left(\omega_{\nu \mathbf{q}}\right) n_{B}\left(-\omega_{\nu^{\prime} \mathbf{p}}\right) \text {, } \\
& R_{\nu \nu^{\prime} \nu^{\prime \prime}}^{(2)}(\mathbf{q}, \mathbf{p})=n_{F}\left(\bar{\xi}_{\nu^{\prime \prime} \mathbf{p}-\mathbf{q}+\mathbf{k}_{N}}\right)\left[n_{B}\left(\omega_{\nu \mathbf{q}}\right)-n_{B}\left(\omega_{\nu^{\prime} \mathbf{p}}\right)\right] \\
& \text { - } n_{B}\left(\omega_{\nu^{\prime} \mathbf{p}}\right) n_{B}\left(-\omega_{\nu \mathbf{q}}\right) \text {, } \\
& R_{\nu \nu^{\prime} \nu^{\prime \prime}}^{(3)}(\mathbf{q}, \mathbf{p})=\left[1-n_{F}\left(\bar{\xi}_{\left.\nu^{\prime \prime} \mathbf{p}-\mathbf{q}+\mathbf{k}_{N}\right)}\right)\right]\left[1+n_{B}\left(\omega_{\nu \mathbf{q}}\right)+n_{B}\left(\omega_{\nu^{\prime} \mathbf{p}}\right)\right] \\
& +n_{B}\left(\omega_{\nu \mathbf{q}}\right) n_{B}\left(\omega_{\nu^{\prime} \mathbf{p}}\right) \text {, }
\end{aligned}
$$

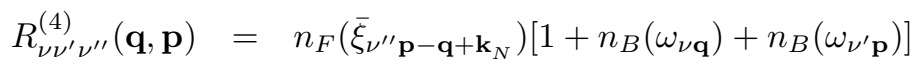

$$
\begin{aligned}
& +n_{B}\left(\omega_{\nu \mathbf{q}}\right) n_{B}\left(\omega_{\nu^{\prime} \mathbf{p}}\right) \text {. }
\end{aligned}
$$


As in the superconducting state, these two self-consistent equations must be solved simultaneously with other self-consistent equations in Eq. (65), then the longitudinal and transverse parts of the electron Green's function in Eqs. (62a) and $(62 \mathrm{~b})$ and the electron spectral function in Eqs. (66a) and (66d) are reduced as,

$$
\begin{aligned}
& G_{L}(\mathbf{k}, \omega)=\frac{1}{8 N} \sum_{\mathbf{p}} \sum_{\mu \nu} Z_{h F A}^{(\mu)} \frac{B_{\nu \mathbf{p}}}{\omega_{\nu \mathbf{p}}}\left(\frac{M_{\mu \nu}^{(1)}(\mathbf{k}, \mathbf{p})}{\omega+\bar{\xi}_{\mu \mathbf{p}-\mathbf{k}}-\omega_{\nu \mathbf{p}}}\right. \\
& \left.+\frac{M_{\mu \nu}^{(2)}(\mathbf{k}, \mathbf{p})}{\omega+\bar{\xi}_{\mu \mathbf{p}-\mathbf{k}}+\omega_{\nu \mathbf{p}}}\right) \\
& G_{T}(\mathbf{k}, \omega)=\frac{1}{8 N} \sum_{\mathbf{p}} \sum_{\mu \nu}(-1)^{\mu+\nu} Z_{h F A}^{(\mu)} \frac{B_{\nu \mathbf{p}}}{\omega_{\nu \mathbf{p}}}\left(\frac{M_{\mu \nu}^{(1)}(\mathbf{k}, \mathbf{p})}{\omega+\bar{\xi}_{\mu \mathbf{p}-\mathbf{k}}-\omega_{\nu \mathbf{p}}}\right. \\
& \left.+\frac{M_{\mu \nu}^{(2)}(\mathbf{k}, \mathbf{p})}{\omega+\bar{\xi}_{\mu \mathbf{p}-\mathbf{k}}+\omega_{\nu \mathbf{p}}}\right) \\
& A_{L}(\mathbf{k}, \omega)=\pi \frac{1}{4 N} \sum_{\mathbf{p}} \sum_{\mu \nu} Z_{h F A}^{(\mu)} \frac{B_{\nu \mathbf{p}}}{\omega_{\nu \mathbf{p}}}\left[M_{\mu \nu}^{(1)}(\mathbf{k}, \mathbf{p}) \delta\left(\omega+\bar{\xi}_{\mu \mathbf{p}-\mathbf{k}}-\omega_{\nu \mathbf{p}}\right)\right. \\
& \left.+M_{\mu \nu}^{(2)}(\mathbf{k}, \mathbf{p}) \delta\left(\omega+\bar{\xi}_{\mu \mathbf{p}-\mathbf{k}}+\omega_{\nu \mathbf{p}}\right)\right] \\
& A_{T}(\mathbf{k}, \omega)=\pi \frac{1}{4 N} \sum_{\mathbf{p}} \sum_{\mu \nu}(-1)^{\mu+\nu} Z_{h F A}^{(\mu)} \frac{B_{\nu \mathbf{p}}}{\omega_{\nu \mathbf{p}}}\left[M_{\mu \nu}^{(1)}(\mathbf{k}, \mathbf{p}) \delta\left(\omega+\bar{\xi}_{\mu \mathbf{p}-\mathbf{k}}-\omega_{\nu \mathbf{p}}\right)\right. \\
& \left.+M_{\mu \nu}^{(2)}(\mathbf{k}, \mathbf{p}) \delta\left(\omega+\bar{\xi}_{\mu \mathbf{p}-\mathbf{k}}+\omega_{\nu \mathbf{p}}\right)\right] .
\end{aligned}
$$

where $M_{\mu \nu}^{(1)}(\mathbf{k}, \mathbf{p})=n_{F}\left(\bar{\xi}_{\mu \mathbf{p}-\mathbf{k}}\right)+n_{B}\left(\omega_{\nu \mathbf{p}}\right), M_{\mu \nu}^{(2)}(\mathbf{k}, \mathbf{p})=1-n_{F}\left(\bar{\xi}_{\mu \mathbf{p}-\mathbf{k}}\right)+$ $n_{B}\left(\omega_{\nu \mathbf{p}}\right)$, then the bonding and antibonding electron spectral functions of the bilayer cuprate superconductors in the normal state are obtained as, $A^{+}(\mathbf{k}, \omega)=$ $\left[A_{L}(\mathbf{k}, \omega)+A_{T}(\mathbf{k}, \omega)\right] / 2$ and $A^{-}(\mathbf{k}, \omega)=\left[A_{L}(\mathbf{k}, \omega)-A_{T}(\mathbf{k}, \omega)\right] / 2$, respectively.

We have performed a calculation for the electron spectral functions of the bilayer cuprate superconductors in the normal state, and the results of the bonding (solid line) and antibonding (dashed line) electron spectral functions at (a) the $[\pi, 0]$ point and (b) $[\pi / 2, \pi / 2]$ point for $t / J=2.5, t^{\prime} / t=0.15$, and $t_{\perp} / t=0.3$ with $T=0.1 J$ at $\delta=0.15$ are plotted in Fig. 12 in comparison with the corresponding experimental data of the bilayer cuprate superconductor $\mathrm{Bi}_{2} \mathrm{Sr}_{2} \mathrm{CaCu}_{2} \mathrm{O}_{8+\delta}$ at the $[\pi, 0]$ point [22] and $[\pi / 2, \pi / 2]$ point [25] in the normal state, respectively. Apparently, (1) there is a double-peak structure in the electron spectral function around the $[\pi, 0]$ point, i.e., the bonding and antibonding quasiparticle peaks around the $[\pi, 0]$ point are located at the different positions, while the bonding and antibonding peaks around the $[\pi / 2, \pi / 2]$ point are located at the same position, which leads to that the bilayer spilitting appears around the $[\pi, 0]$ point, and is absent from the vicinity of the $[\pi / 2, \pi / 2]$ point; (2) The position of the antibonding peak at the $[\pi, 0]$ point is more closer to the Fermi energy than these for the bonding peak; (3) In analogy to the single layer case 34 discussion in section 3, both positions of the quasiparti- 

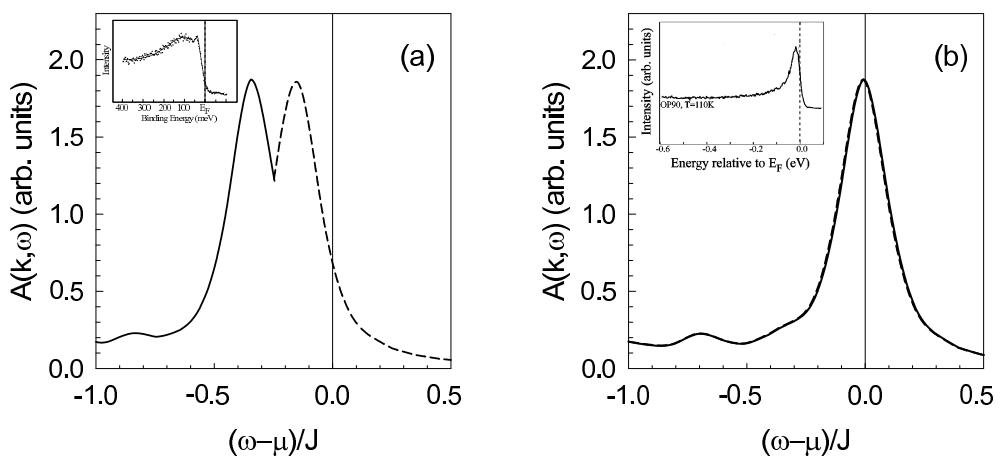

Figure 1.12: The bonding (solid line) and antibonding (dashed line) electron spectral functions of the bilayer cuprate superconductors in the normal state as a function of energy at (a) the $[\pi, 0]$ point and (b) $[\pi / 2, \pi / 2]$ point for $t / J=2.5$, $t^{\prime} / t=0.15$, and $t_{\perp} / t=0.3$ with $T=0.1 J$ at $\delta=0.15$. Inset: the corresponding experimental data of the bilayer cuprate superconductor $\mathrm{Bi}_{2} \mathrm{Sr}_{2} \mathrm{CaCu}_{2} \mathrm{O}_{8+\delta}$ at the $[\pi, 0]$ point and $[\pi / 2, \pi / 2]$ point in the normal state taken from Refs. [22] and [25], respectively.

cle peaks from the bonding and antibonding electron spectral functions at the $[\pi, 0]$ and $[\pi / 2, \pi / 2]$ points are below the Fermi energy, but the positions of the peaks at the $[\pi / 2, \pi / 2]$ point are more closer to the Fermi energy, which indicates that the lowest energy states are located at the $[\pi / 2, \pi / 2]$ point, in other words, the low energy spectral weight with the majority contribution to the low-energy properties of the bilayer cuprate superconductors in the normal state comes from the $[\pi / 2, \pi / 2]$ point, in qualitative agreement with the ARPES experimental data on the bilayer cuprate superconductors in the normal state [3, 22, 25, 74, 75, 76. In the above calculations, we also find that the doublepeak structure in the electron spectral functions around the $[\pi, 0]$ point is closely related to the interlayer hopping form in Eq. (49). With decreasing the values of $t_{\perp}$ and $J_{\perp}$, the distance between the bonding and antibonding peaks in the electron spectral functions decreases. When $t_{\perp}=0$ and $J_{\perp}=0$, we find that the transverse part of the dressed holon Green's functions in Eq. (70b) (then the transverse part of the electron Green's functions in Eq. (73b) and transverse part of the electron spectral functions in Eq. (73d)) is equal to the zero. In this case, the bonding electron spectral function is exactly same as the antibonding electron spectral function, then the electron spectral functions are reduced to the single layer case [34].

For a better understanding of the physical properties of the electron spectrum of the bilayer cuprate superconductors in the normal state, we have studied the electron spectrum at different doping concentrations, and the result of the electron spectral functions at $[\pi, 0]$ point for $t / J=2.5, t^{\prime} / t=0.15$, and $t_{\perp} / t=0.3$ with $T=0.1 J$ at $\delta=0.09$ (solid line), $\delta=0.12$ (dashed line), and $\delta=0.15$ (dotted line) are plotted in Fig. 13 in comparison with the correspond- 


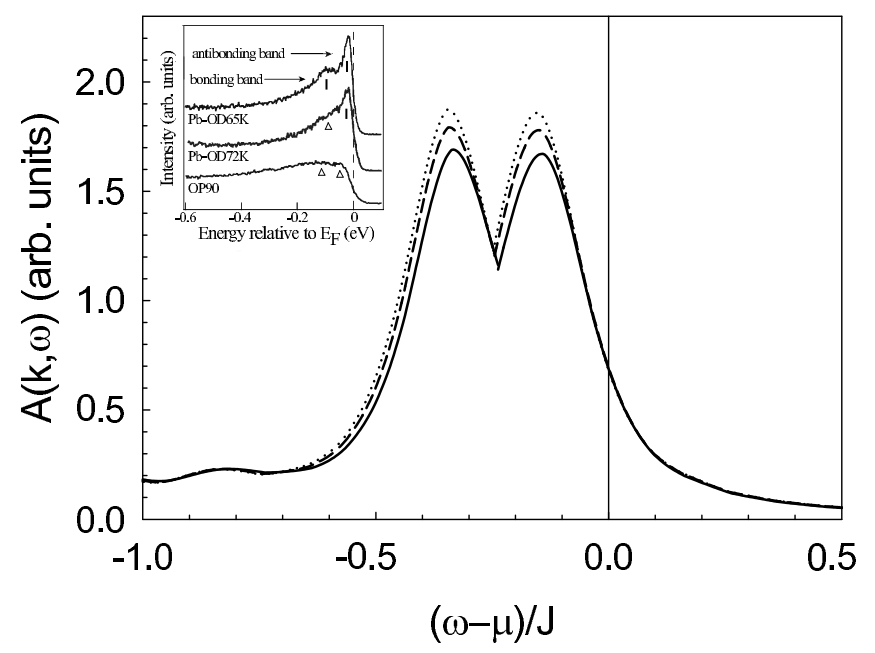

Figure 1.13: The electron spectral functions of the bilayer cuprate superconductors at $[\pi, 0]$ point in the normal state for $t / J=2.5, t^{\prime} / t=0.15$, and $t_{\perp} / t=0.3$ with $T=0.1 J$ at $\delta=0.09$ (solid line), $\delta=0.12$ (dashed line), and $\delta=0.15$ (dotted line). Inset: the corresponding experimental data of the bilayer cuprate superconductor $\mathrm{Bi}_{2} \mathrm{Sr}_{2} \mathrm{CaCu}_{2} \mathrm{O}_{8+\delta}$ in the normal state taken from Ref. [25]

ing experimental data of the bilayer cuprate superconductor $\mathrm{Bi}_{2} \mathrm{Sr}_{2} \mathrm{CaCu}_{2} \mathrm{O}_{8+\delta}$ in the normal state [25], which indicates that with increasing the doping concentration, both bonding and antibonding quasiparticle peaks become sharper, and the spectral weights of these peaks increase in intensity. Furthermore, we have also discussed the temperature dependence of the electron spectrum of the bilayer cuprate superconductors in the normal state, and the result of the electron spectral functions at $[\pi, 0]$ point for $t / J=2.5, t^{\prime} / t=0.15$, and $t_{\perp} / t=0.3$ at $\delta=0.15$ with $T=0.1 J$ (solid line), $T=0.05 J$ (dashed line), and $T=0.01 J$ (dotted line) are plotted in Fig. 14 in comparison with the corresponding experimental data of the bilayer cuprate superconductor $\mathrm{Bi}_{2} \mathrm{Sr}_{2} \mathrm{CaCu}_{2} \mathrm{O}_{8+\delta}$ in the normal state [22, it is shown obviously that both bonding and antibonding spectral weights are suppressed with increasing temperatures. These results are also qualitatively consistent with the ARPES experimental results on the bilayer cuprate superconductors in the normal state [3, 22, 25, 74, 75, 76].

For considering the quasiparticle dispersion of the bilayer cuprate superconductors in the normal state, we have made a series of calculations for both bonding and antibonding electron spectral functions at different momenta, and find that the lowest energy peaks in the normal state are well defined at all momenta. In particular, the positions of both bonding and antibonding quasiparticle peaks as a function of energy $\omega$ for momentum $\mathbf{k}$ in the vicinity of the $[\pi, 0]$ point are almost not changeable as in the single layer case, which leads to the unusual quasiparticle dispersion around the $[\pi, 0]$ point. To show this broad 


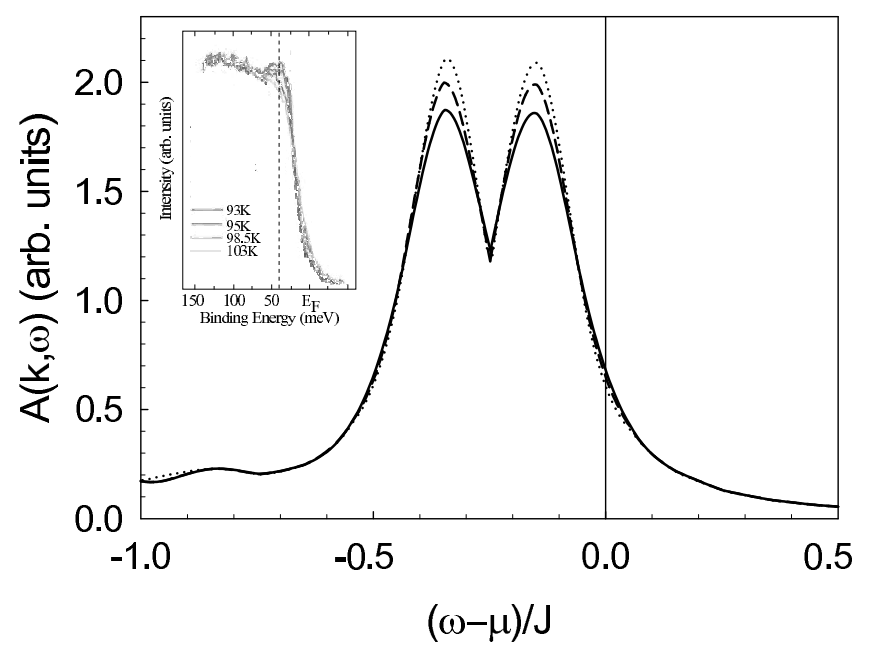

Figure 1.14: The electron spectral functions of the bilayer cuprate superconductors at $[\pi, 0]$ point in the normal state for $t / J=2.5, t^{\prime} / t=0.15$, and $t_{\perp} / t=0.3$ at $\delta=0.15$ with $T=0.1 J$ (solid line), $T=0.05 J$ (dashed line), and $T=0.01 J$ (dotted line). Inset: the corresponding experimental data of the bilayer cuprate superconductor $\mathrm{Bi}_{2} \mathrm{Sr}_{2} \mathrm{CaCu}_{2} \mathrm{O}_{8+\delta}$ in the normal state taken from Ref. [22].

feature clearly, we plot the positions of the lowest energy quasiparticle peaks in the bonding and antibonding electron spectra as a function of momentum along the high symmetry directions with $T=0.1 J$ at $\delta=0.15$ for $t / J=2.5$, $t^{\prime} / t=0.15$, and $t_{\perp} / t=0.3$ in Fig. 15. For comparison, the corresponding result from the tight binding fit to the experimental data of the bilayer cuprate superconductor $\mathrm{Bi}_{2} \mathrm{Sr}_{2} \mathrm{CaCu}_{2} \mathrm{O}_{8+\delta}$ in the normal state [75] is also shown in Fig. 15 (inset). It is shown that in analogy to the single layer case [34, both electron bonding and antibonding quasiparticles around the $[\pi, 0]$ point disperse very weakly with momentum, and then the two main flat bands appear, while the Fermi energy is only slightly above these flat bands. Moreover, this bilayer energy band splitting reaches its maximum at the $[\pi, 0]$ point, in qualitative agreement with these obtained from the ARPES experimental measurements on the bilayer cuprate superconductors in the normal state [3, 74, 75, 76].

In comparison with the single layer case [35] discussed in section 3, we therefore find that the essential physics of the double-peak structure of the electron spectral function of the bilayer cuprate superconductors around the $[\pi, 0]$ point in the normal state is dominated by the bilayer interaction. Since the full electron Green's function in the bilayer cuprate superconductors in the normal state is divided into the longitudinal and transverse parts, respectively, due to the bilayer interaction, then these longitudinal and transverse Green's functions (then the bonding and antibonding electron spectral functions and corresponding quasiparticle dispersions) are obtained beyond the mean-field approximation 


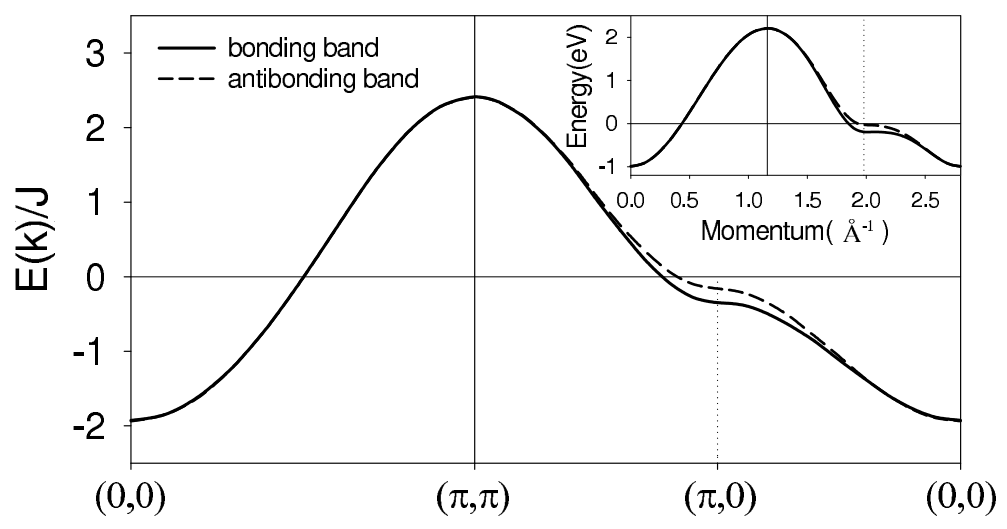

Figure 1.15: The positions of the lowest energy quasiparticle peaks in the bonding (solid line) and antibonding (dashed line) electron spectra as a function of momentum with $T=0.1 J$ at $\delta=0.15$ for $t / J=2.5, t^{\prime} / t=0.15$, and $t_{\perp} / t=0.3$. Inset: the corresponding result from the tight binding fit to the experimental data of the bilayer cuprate superconductor $\mathrm{Bi}_{2} \mathrm{Sr}_{2} \mathrm{CaCu}_{2} \mathrm{O}_{8+\delta}$ in the normal state taken from Ref. [75].

by considering the dressed holon fluctuation due to the spin pair bubble, therefore the nature of the bonding and antibonding electron spectral functions of the bilayer cuprate superconductors in the normal state is closely related to the strong interaction between the dressed holons (then electron quasiparticles) and collective magnetic excitations. In this case, the single-particle hoppings in the bilayer $t-J$ model are strongly renormalized by the magnetic interaction as in the single layer case discussed in section 3. As a consequence, both bonding and antibonding quasiparticle bandwidths are reduced to the order of (a few) $J$, and then the energy scales of both bonding and antibonding quasiparticle energy bands are controlled by the magnetic interaction. These renormalizations for both bonding and antibonding energy bands are then responsible for the unusual bonding and antibonding electron quasiparticle spectra and production of the two main flat bands around the $[\pi, 0]$ point.

\subsection{Summary and discussions}

In the charge-spin separation fermion-spin theory [29], the physical electron is decoupled completely as the gauge invariant dressed holon and spin, with the dressed holon keeps track of the charge degree of freedom together with some effects of the spin configuration rearrangements due to the presence of the doped hole itself, while the spin operator keeps track of the spin degree of freedom. In particular, we have shown that the charge-spin separation fermion-spin transformation in Eq. (9) is a natural representation for the constrained electron under the decoupling scheme. The advantage of this charge-spin separation 
fermion-spin theory is that the electron local constraint for single occupancy is satisfied in analytical calculations. Moreover, both dressed holon and spin are gauge invariant, and in this sense, they are real and can be interpreted as the physical excitations [29]. Within this charge-spin separation fermion-spin theory, we have developed a kinetic energy driven superconducting mechanism [30], where the dressed holons interact occurring directly through the kinetic energy by exchanging the spin excitations, leading to a net attractive force between the dressed holons, then the electron Cooper pairs originating from the dressed holon pairing state are due to the charge-spin recombination, and their condensation reveals the superconducting ground-state. Furthermore, this superconducting state is controlled by both superconducting gap parameter and quasiparticle coherence, then the maximal superconducting transition temperature occurs around the optimal doping, and decreases in both underdoped and overdoped regimes 31 , 32.

In summary, we have given a brief review of the electronic structure of the single layer and bilayer cuprate superconductors in both superconducting and normal states [32, 34, 35, 36, 37. Within the framework of the kinetic energy driven superconductivity, we show the spectral weight in both superconducting and normal states increases with increasing doping, and decreases with increasing temperatures [32, 33, 34, 35, 36, 37. In the normal state, although the lowest energy states are located at the $[\pi / 2, \pi / 2]$ point, the quasiparticle dispersion exhibits the unusual flat band behavior around the $[\pi, 0]$ point below the Fermi energy. In corresponding to this unusual normal state flat band behavior around the $[\pi, 0]$ point, the superconducting quasiparticles around the $[\pi, 0]$ disperse very weakly with momentum. In particular, we show that one of the universal features is that the d-wave superconducting state in cuprate superconductors is the conventional BCS like, so that the basic BCS formalism with the d-wave gap function is still valid in quantitatively reproducing of the doping dependence of the superconducting gap parameter, the doping dependence of the superconducting transition temperature, and the low energy electronic structure of cuprate superconductors [32, 33, 35, 37, although the pairing mechanism is driven by the kinetic energy by exchanging spin excitations, and other exotic properties are beyond the BCS formalism. Furthermore, we have shown that the observed peak-dip-hump structure in the bilayer cuprate superconductors in the superconducting state and double-peak structure in the normal state around the $[\pi, 0]$ point is mainly caused by the bilayer splitting, with the superconducting quasiparticle peak in the superconducting state (lower energy quasiparticle peak in the normal state) being related to the antibonding component, and the hump in the superconducting state (higher energy quasiparticle peak in the normal state) being formed by the bonding component. In this sense, the peak-dip-hump structure in the bilayer cuprate superconductors in the superconducting state is unrelated to superconductivity. Our these theoretical results [32, 33, 34, 35, 36, 37, also show that the striking behavior of the electronic structure in cuprate superconductors is intriguingly related to the strong coupling between the electron quasiparticles and collective magnetic excitations. Based on the kinetic energy driven superconductivity, we [79] have 
discussed the electronic structure of the electron-doped cuprate superconductors, and the results show explicitly that although the electron-hole asymmetry is observed in the phase diagram, the electronic structure of the electron-doped cuprates in the superconducting state is similar to that in the hole-doped case.

\section{Acknowledgements}

This work was supported by the National Natural Science Foundation of China under Grant Nos. 90403005 and 10774015, and the funds from the Ministry of Science and Technology of China under Grant Nos. 2006CB601002 and 2006CB921300. 


\section{Bibliography}

[*] The present address: Department of Physics, Capital Normal University, Beijing 100037, China.

[1] J. G. Bednorz and K.A. Müller, Z. Phys. B 64, 189 (1986).

[2] See, e.g., M. A.Kastner, R. J. Birgeneau, G. Shirane, and Y. Endoh, Rev. Mod. Phys. 70, 897 (1998), and references therein.

[3] See, e.g., Z. X. Shen, and D. S. Dessau, Phys. Rep. 253, 1 (1995), and referenes therein.

[4] See, e.g., A. Damascelli, Z. Hussain, and Z. X. Shen, Rev. Mod. Phys. 75, 473 (2003), and references therein.

[5] See, e.g., J. Campuzano, M. Norman, and M. Randeira, in Physics of Superconductors, vol. II, edited by K. Bennemann and J. Ketterson (Springer, Berlin Heidelberg New York, 2004), p. 167, and references therein.

[6] P. W. Anderson, Science 235, 1196 (1987); P. W. Anderson, in Frontiers and Borderlines in Many Particle Physics, edited by R. A. Broglia and J. R. Schrieffer (North-Holland, Amsterdam, 1987), p. 1.

[7] P. W. Anderson, Phys. Rev. Lett. 67, 2092 (1991); Science 288, 480 (2000); Physica C 341-348, 9 (2000); cond-mat/0108522 (unpublished).

[8] P. W. Anderson, arXiv:0709.0656 (unpublished).

[9] R. B. Laughlin, Phys. Rev. Lett. 79, 1726 (1997); J. Low. Tem. Phys. 99, 443 (1995).

[10] Z. X. Shen, W. E. Spicer, D. M. King, D. S. Dessau, and B. O. Wells, Science 267, 343 (1995); C. Kim, P. J. White, Z. X. Shen, T. Tohyama, Y. Shibata, S. Maekawa, B. O. Wells, Y. J. Kim, R. J. Birgeneau, and M. A. Kastner, Phys. Rev. Lett. 80, 4245 (1998).

[11] D. S. Dessau, Z. X. Shen, D. M. King, D. S. Marshall, L. W. Lombardo, P. H. Dickinson, A. G. Loeser, J. DiCarlo, C. H. Park, A. Kapitulnik, and W. E. Spicer, Phys. Rev. Lett. 71, 2781 (1993). 
[12] B. O. Wells, Z. X. Shen, A. Matsuura, D. M. King, M. A. Kastner, M. Greven, and R. J. Birgeneau, Phys. Rev. Lett. 74, 964 (1995); D. S. Marshall, D. S. Dessau, A. G. Loeser, C. H. Park, A. Y. Matsuura, J. N. Eckstein, I. Bozovic, P. Fournier, A. Kapitulnik, W. E. Spicer, and Z. X. Shen, Phys. Rev. Lett. 76, 4841 (1996).

[13] H. Ding, J. R. Engelbrecht, Z. Wang, J. C. Campuzano, S. C. Wang, H. B. Yang, R. Rogan, T. Takahashi, K. Kadowaki, and D. G. Hinks, Phys. Rev. Lett. 87, 227001 (2001).

[14] H. Matsui, T. Sato, T. Takahashi, S. C. Wang, H. B. Yang, H. Ding, T. Fujii, T. Watanabe, and A. Matsuda, Phys. Rev. Lett. 90, 217002 (2003).

[15] J. C. Campuzano, H. Ding, M. R. Norman, M. Randeira, A. F. Bellman, T. Yokoya, T. Takahashi, H. Katayama-Yoshida, T. Mochiku, and K. Kadowaki, Phys. Rev. B 53, R14737 (1996).

[16] K. Yamada, C.H. Lee, K. Kurahashi, J. Wada, S. Wakimoto, S. Ueki, H. Kimura, Y. Endoh, S. Hosoya, G. Shirane, R. J. Birgeneau, M. Greven, M.A. Kastner, and Y. J. Kim, Phys. Rev. B 57, 6165 (1998).

[17] P. Dai, H. A. Mook, R. D. Hunt, and F. Dog̃an, Phys. Rev. B 63, 054525 (2001); P. Bourges, B. Keimer, S. Pailhés, L. P. Regnault, Y. Sidis, and C. Ulrich, Physica C 424, 45 (2005).

[18] M. Arai, T. Nishijima, Y. Endoh, T. Egami, S. Tajima, K. Tomimoto, Y. Shiohara, M. Takahashi, A. Garret, and S. M. Bennington, Phys. Rev. Lett. 83, 608 (1999); S. M. Hayden, H. A. Mook, P. Dai, T. G. Perring, and F. Dog̃an, Nature 429, 531 (2004); C. Stock, W. J. Buyers, R. A. Cowley, P. S. Clegg, R. Coldea, C. D. Frost, R. Liang, D. Peets, D. Bonn, W. N. Hardy, and R. J. Birgeneau, Phys. Rev. B 71, 024522 (2005).

[19] J. R. Schrieffer, Theory of Superconductivity, Benjamin, New York, 1964.

[20] D. S. Dessau, B. O. Wells, Z. X. Shen, W. E. Spicer, A. J. Arko, R. S. List, D. B. Mitzi, and A. Kapitulnik, Phys. Rev. Lett. 66, 2160 (1991); Y. Hwu, L. Lozzi, M. Marsi, S. La Rosa, M. Winokur, P. Davis, M. Onellion, H. Berger, F. Gozzo, F. Lévy, and G. Margaritondo, Phys. Rev. Lett. 67, 2573 (1991).

[21] Mohit Randeria, Hong Ding, J. C. Campuzano, A. Bellman, G. Jennings, T. Yokoya, T. Takahashi, H. Katayama-Yoshida, T. Mochiku, and K. Kadowaki, Phys. Rev. Lett. 74, 4951 (1995); H. Ding, T. Yokoya, J. C. Campuzano, T. Takahashi, M. Randeria, M. R. Norman, T. Mochiku, K. Kadowaki, and J. Giapintzakis, Nature 382, 51 (1996).

[22] A. V. Fedorov, T. Valla, P. D. Johnson, Q. Li, G. D. Gu, and N. Koshizuka, Phys. Rev. Lett. 82, 2179 (1999). 
[23] D. H. Lu, D. L. Feng, N. P. Armitage, K. M. Shen, A. Damascelli, C. Kim, F. Ronning, Z. X. Shen, D. A. Bonn, R. Liang, W. N. Hardy, A. I. Rykov, and S. Tajima, Phys. Rev. Lett. 86, 4370 (2001).

[24] T. Sato, H. Matsui, S. Nishina, T. Takahashi, T. Fujii, T. Watanabe, and A. Matsuda, Phys. Rev. Lett. 89, 067005 (2002); D. L. Feng, A. Damascelli, K. M. Shen, N. Motoyama, D. H. Lu, H. Eisaki, K. Shimizu, J.-i. Shimoyama, K. Kishio, N. Kaneko, M. Greven, G. D. Gu, X. J. Zhou, C. Kim, F. Ronning, N. P. Armitage, and Z. X Shen, Phys. Rev. Lett. 88, 107001 (2002).

[25] D. L. Feng, C. Kim, H. Eisaki, D. H. Lu, A. Damascelli, K. M. Shen, F. Ronning, N. P. Armitage, N. Kaneko1, M. Greven, J.-i. Shimoyama, K. Kishio, R. Yoshizaki, G. D. Gu, and Z. X. Shen, Phys. Rev. B 65, 220501(R) (2002); A. A. Kordyuk, S. V. Borisenko, M. S. Golden, S. Legner, K. A. Nenkov, M. Knupfer, J. Fink, H. Berger, L. Forró, and R. Follath, Phys. Rev. B 66, 014502 (2002); Y.-D. Chuang, A. D. Gromko, A. V. Fedorov, Y. Aiura, K. Oka, Yoichi Ando, D. S. Dessau, cond-mat/0107002 (unpublished).

[26] K. Terashima, H. Matsui, D. Hashimoto, T. Sato, T. Takahashi, H. Ding, T. Yamamoto and K. Kadowaki, Nature Phys. 2, 27 (2006).

[27] C. Gros, Phys. Rev. B 38, 931 (1988); T. K. Lee and Shiping Feng, Phys. Rev. B 38, 11809 (1988); S. Sorella, G. B. Martins, F. Becca, C. Gazza, L. Capriotti, A. Parola, and E. Dagotto, Phys. Rev. Lett. 88, 117002 (2002); D. Poilblanc and D. J. Scalapino, Phys. Rev. B 66, 052513 (2002); D. Eichenberger and D. Baeriswyl, arXiv:0708.2795 (unpublished); L. Spanu, M. Lugas, F. Becca, and S. Sorella, arXiv:0709.2850 (unpublished).

[28] See, e.g., E. Dagotto, Rev. Mod. Phys. 66, 763 (1994); Th. Maier, M. Jarrell, T. Pruschke, and M. H. Hettler, Rev. Mod. Phys. 77, 1027 (2005).

[29] Shiping Feng, Jihong Qin, and Tianxing Ma, J. Phys. Condens. Matter 16, 343 (2004); Shiping Feng, Tianxing Ma, and Jihong Qin, Mod. Phys. Lett. B 17, 361 (2003).

[30] Shiping Feng, Phys. Rev. B 68, 184501 (2003).

[31] Shiping Feng, Tianxing Ma, and Huaiming Guo, Physica C 436, 14 (2006); Shiping Feng and Huaiming Guo, in it Proceedings of the International Conference on Materials and Mechanisms of Superconductivity and High Temperature Superconductors VIII, Dresden, Germany, 2006 [Physica C 460-462, 230 (2007)]; Tianxing Ma, Huaiming Guo, and Shiping Feng, Mod. Phys. Lett. B 18, 895 (2004).

[32] Shiping Feng and Tianxing Ma, Phys. Lett. A 350, 138 (2006).

[33] Shiping Feng and Tianxing Ma, in Superconductivity Research Horizons, edited by Eugene H. Peterson (Nova Science Publishers, Nrw York, 2007) chapter 5, pp. 129-158, and references therein. 
[34] Huaiming Guo and Shiping Feng, Phys. Lett. A 355, 473 (2006).

[35] Huaiming Guo and Shiping Feng, Phys. Lett. A 361, 382 (2007).

[36] Yu Lan, Jihong Qin, and Shiping Feng, Phys. Rev. B 75, 134513 (2007).

[37] Yu Lan, Jihong Qin, and Shiping Feng, Phys. Rev. B 76, 014533 (2007).

[38] F. D. M. Haldane, Phys. Rev. Lett. 45, 1358 (1980); Phys. Lett. A 81, 153 (1981); J. Solyom, Adv. Phys. 28, 201(1979).

[39] H. Yokoyama and M. Ogata, Phys. Rev. Lett. 67, 3610 (1991); F.F. Assaad and D. Würtz, Phys. Rev. B 44, 2681 (1991).

[40] C. Kim, A. Y. Matsuura, Z. X. Shen, N. Motoyama, H. Eisaki, S. Uchida, T. Tohyama, and S. Maekawa, Phys. Rev. Lett. 77, 4054 (1996); B. J. Kim, H. Koh, E. Rotenberg, S.-J. Oh, H. Eisaki, N. Motoyama, S. Uchida, T. Tohyama, S. Maekawa, Z.-X. Shen and C. Kim, Nature Phys. 2, 397 (2006).

[41] See, e.g., S. Maekawa and T. Tohyama, Rep. Prog. Phys. 64, 383 (2001), and references therein.

[42] Joseph Orenstein, G. A. Thomas, A. J. Millis, S. L. Cooper, D. H. Rapkine, T. Timusk, L. F. Schneemeyer, and J. V. Waszczak, Phys. Rev. B 42, 6342 (1990); D.B. Tanner and T. Timusk, in Physical Properties of High Temperature Superconductors III, edited by D.M. Ginsberg (World Scientific, Singapore, 1992), p. 363, and references therein.

[43] S. Uchida, Physca C 282-287, 12 (1997), and references therein.

[44] H. Takagi, B. Batlogg, H. L. Kao, J. Kwo, R. J. Cava, J. J. Krajewski, and W. F. Peck, Jr., Phys. Rev. Lett. 69, 2975 (1992).

[45] Yoichi Ando, A. N. Lavrov, Seiki Komiya, Kouji Segawa, and X. F. Sun, Phys. Rev. Lett. 87, 017001 (2001); Yoichi Ando, Kouji Segawa, A.N. Lavrov, Seiki Komiya, J. Low Temperature Phys. 131, 793 (2003).

[46] R. W. Hill, Cyril Proust, Louis Taillefer, P. Fournier and R. L. Greene, Nature 414, 711 (2001).

[47] C. Gros, R. Joynt, and T.M. Rice, Phys. Rev. B 36, 381 (1987).

[48] L. Zhang, J. K. Jain, and V. J. Emery, Phys. Rev. B 47, 3368 (1993); Shiping Feng, J. B. Wu, Z. B. Su, and L. Yu, Phys. Rev. B 4715192 (1993).

[49] Shiping Feng, Z.B. Su, and L. Yu, Phys. Rev. B 49, 2368 (1994); Mod. Phys. Lett. B 7, 1013 (1993).

[50] P.B. Wiegmann, Phys. Rev. Lett. 60, 821 (1988). 
[51] George B. Martins, Robert Eder, and Elbio Dagotto, Phys. Rev. B 60, R3716 (1999); G. B. Martins, J. C. Xavier, C. Gazza, M. Vojta, and E. Dagotto, Phys. Rev. B 63, 014414 (2000); G. B. Martins, C. Gazza, J. C. Xavier, A. Feiguin, and E. Dagotto, Phys. Rev. Lett. 84, 5844 (2000).

[52] N. M. Plakida, Condens. Matter Phys. 5, 707 (2002).

[53] K. Tanaka, T. Yoshida, A. Fujimori, D. H. Lu, Z. X. Shen, X. J. Zhou, H. Eisaki, Z. Hussain, S. Uchida, Y. Aiura, K. Ono, T. Sugaya, T. Mizuno, and I. Terasaki, Phys. Rev. B 70, 092503 (2004).

[54] See, e.g., C.C. Tsuei and J.P. Kirtley, Rev. Mod. Phys. 72, 969 (2000).

[55] Z. X. Shen, D. S. Dessau, B. O. Wells, D. M. King, W. E. Spicer, A. J. Arko, D. Marshall, L. W. Lombardo, A. Kapitulnik, P. Dickinson, S. Doniach, J. DiCarlo, A. G. Loeser, and C. H. Park, Phys. Rev. Lett. 70, 1553 (1993); H. Ding, M. R. Norman, J. C. Campuzano, M. Randeria, A. F. Bellman, T. Yokoya, T. Takahashi, T. Mochiku, and K. Kadowaki, Phys. Rev. B 54, R9678 (1996).

[56] I. Bozovic, G. Logvenov, M. A. J. Verhoeven, P. Gaputo, E. Goldobin, and T. H. Geballe, Nature 422, 873 (2003).

[57] Shiping Feng and Yun Song, Phys. Rev. B 55, 642 (1997).

[58] J. Kondo and K. Yamaji, Prog. Theor. Phys. 47, 807 (1972).

[59] Shiping Feng and Zhongbing Huang, Phys. Lett. A 232, 293 (1997); Feng Yuan, Jihong Qin, Shiping Feng, and W. Y. Chen, Phys. Rev. B 67, 134505 (2003); Shiping Feng, Feng Yuan, and Weiqiang Yu, Eur. Phys. J. B 15, 607(2000); Shiping Feng, Feng Yuan, Weiqiang Yu, and Pengpeng Zhang, Phys. Rev. B 60, 7565(1999).

[60] J. Graf, G.-H. Gweon, K. McElroy, S. Y. Zhou, C. Jozwiak, E. Rotenberg, A. Bill, T. Sasagawa, H. Eisaki, S. Uchida, H. Takagi, D.-H. Lee, and A. Lanzara, Phys. Rev. Lett. 98, 067004 (2007); B. P. Xie, K. Yang, D. W. Shen, J. F. Zhao, H. W. Ou, J. Wei, S. Y. Gu, M. Arita, S. Qiao, H. Namatame, M. Taniguchi, N. Kaneko, H. Eisaki, K. D. Tsuei, C. M. Cheng, I. Vobornik, J. Fujii, G. Rossi, Z. Q. Yang, and D. L. Feng, Phys. Rev. Lett. 98, 147001 (2007); K. Byczuk, M. Kollar, K. Held, Y.-F. Yang, I. A. Nekrasov, Th. Pruschke and D. Vollhardt, Nature Phys. 3, 168 (2007).

[61] G. M. Eliashberg, Sov. Phys. JETP 11, 696 (1960); D. J. Scalapino, J. R. Schrieffer, and J. W. Wilkins, Phys. Rev. 148, 263 (1966).

[62] S. Huefner, M. A. Hossain, A. Damascelli, G. A. Sawatzky, arXiv:0706.4282 (unpublished).

[63] See, e.g., J.L. Tallon, J.W. Loram, J.R. Cooper, C. Panagopoulos, and C. Bernhard, Phys. Rev. B 68, 180501 (R) (2003). 
[64] J. C. Campuzano, H. Ding, M. R. Norman, H. M. Fretwell, M. Randeira, A. Kaminski, J. Mesot, T. Takeuchi, T. Sato, T. Yokoya, T. Takahashi, T. Mochiku, K. Kadowaki, P. Guptasarma, D. G. Hinks, Z. Konstantinovic, Z. Z. Li, and H. Raffy, Phys. Rev. Lett. 83, 3709 (1999); M. R. Norman, H. Ding, J. C. Campuzano, T. Takeuchi, M. Randeria, T. Yokoya, T. Takahashi, T. Mochiku, and K. Kadowaki, Phys. Rev. Lett. 79, 3506 (1997).

[65] Y. J. Uemura, G. M. Luke, B. J. Sternlieb, J. H. Brewer, J. F. Carolan, W. N. Hardy, R. Kadono, J. R. Kempton, R. F. Kiefl, S. R. Kreitzman, P. Mulhern, T. M. Riseman, D. L. Williams, B. X. Yang, S. Uchida, H. Takagi, J. Gopalakrishnan, A. W. Sleight, M. A. Subramanian, C. L. Chien, M. Z. Cieplak, G. Xiao, V. Y. Lee, B. W. Statt, C. E. Stronach, W. J. Kossler, and X. H. Yu, Phys. Rev. Lett. 62, 2317 (1989); Y. J. Uemura, L. P. Le, G. M. Luke, B. J. Sternlieb, W. D. Wu, J. H. Brewer, T. M. Riseman, C. L. Seaman, M. B. Maple, M. Ishikawa, D. G. Hinks, J. D. Jorgensen, G. Saito, and H. Yamochi, Phys. Rev. Lett. 66, 2665 (1991).

[66] T. Sato, T. Kamiyama, Y. Naitoh, T. Takahashi, I. Chong, T. Terashima, and M. Takano, Phys. Rev. B 63, 132502 (2001); T. Sato, T. Kamiyama, T. Takahashi, J. Mesot, A. Kaminski, J. C. Campuzano, H. M. Fretwell, T. Takeuchi, H. Ding, I. Chong, T. Terashima, and M. Takano, Phys. Rev. B 64, 054502 (2001).

[67] X. J. Zhou, T. Yoshida, S. A. Kellar, P. V. Bogdanov, E. D. Lu, A. Lanzara, M. Nakamura, T. Noda, T. Kakeshita, H. Eisaki, S. Uchida, A. Fujimori, Z. Hussain, and Z. X. Shen, Phys. Rev. Lett. 86, 5578 (2001); T. Yoshida, X. J. Zhou, T. Sasagawa, W. L. Yang, P. V. Bogdanov, A. Lanzara, Z. Hussain, T. Mizokawa, A. Fujimori, H. Eisaki, Z. X. Shen, T. Kakeshita, and S. Uchida, Phys. Rev. Lett. 91, 027001 (2003); X. J. Zhou, T. Yoshida, D.-H. Lee, W. L. Yang, V. Brouet, F. Zhou, W. X. Ti, J. W. Xiong, Z. X. Zhao, T. Sasagawa, T. Kakeshita, H. Eisaki, S. Uchida, A. Fujimori, Z. Hussain, and Z. X. Shen, Phys. Rev. Lett. 92, 187001 (2004); K. Terashima, H. Matsui, T. Sato, T. Takahashi, M. Kofu, and K. Hirota, Phys. Rev. Lett. 99, 017003 (2007).

[68] T. Takeuchi, T. Yokya, S. Shin, K. Jinno, M. Matsuura, T. Kondo, H. Ikuta, and U. Mizutani, J. Electron Spectrosc. Relat. Phenom. 114-116, 629 (2001).

[69] L. D. Landau, Sov. Phys. JETP 3, 920 (1956); Sov. Phys. JETP 5, 101 (1957); Sov. Phys. JETP 8, 70 (1959).

[70] T. M. Rice, Physica C 282-287, xix (1997).

[71] Huaiming Guo and Shiping Feng, In Proceedings of the Sixth International Conference on New Theories, Discoveries and Applications of Superconductors and Related Materials, Sydney, Australia, January 9-11, 2007 [Int. J. Mod. Phys. B 21, 3108 (2007)]. 
[72] Cody P. Nave, Dmitri A. Ivanov, and Patrick A. Lee, Phys. Rev. B 73, 104502 (2006); Samuel Bieri and Dmitri Ivanov, Phys. Rev. B 75, 035104 (2007); S. Yunoki, Phys. Rev. B 74, 180504(R) (2006); Hong-Yu Yang, Fan Yang, Yong-Jin Jiang, and Tao Li, cond-mat/0604488 (unpublished).

[73] Takeshi Kondo, Tsunehiro Takeuchi, Adam Kaminski, Syunsuke Tsuda, and Shik Shin, Phys. Rev. Lett. 98, 267004 (2007).

[74] D. L. Feng, N. P. Armitage, D. H. Lu, A. Damascelli, J. P. Hu, P. Bogdanov, A. Lanzara, F. Ronning, K. M. Shen, H. Eisaki, C. Kim, Z. X. Shen, J.-i. Shimoyama, and K. Kishio, Phys. Rev. Lett. 86, 5550 (2001).

[75] A. A. Kordyuk, S. V. Borisenko, M. Knupfer, and J. Fink, Phys. Rev. B 67, 064504 (2003); A. A. Kordyuk and S. V. Borisenko, Low Temp. Phys. 32, 298 (2006).

[76] Y.-D. Chuang, A. D. Gromko, A. Fedorov, Y. Aiura, K. Oka, Y. Ando, H. Eisaki, S. I. Uchida, and D. S. Dessau, Phys. Rev. Lett. 87, 117002 (2001); P. V. Bogdanov, A. Lanzara, X. J. Zhou, S. A. Kellar, D. L. Feng, E. D. Lu, H. Eisaki, J.-I. Shimoyama, K. Kishio, Z. Hussain, and Z. X. Shen, Phys. Rev. B 64, 180505(R) (2001).

[77] S. V. Borisenko, A. A. Kordyuk, T. K. Kim, S. Legner, K. A. Nenkov, M. Knupfer, M. S. Golden, J. Fink, H. Berger, and R. Follath, Phys. Rev. B 66, 140509(R) (2002).

[78] O. K. Anderson, A. I. Liechtenstein, O. Jepsen, and F. Paulsen, J. Phys. Chem. Solids 56, 1573 (1995); A. I. Liechtenstein, O. Gunnarsson, O. K. Anderson, and R. M. Martin, Phys. Rev. B 54, 12505 (1996); S. Chakarvarty, A. Sudbo, P. W. Anderson, and S. Strong, Science 261, 337 (1993).

[79] Li Cheng, Huaiming Guo, and Shiping Feng, Phys. Lett. A 366, 137 (2007). 